\title{
Finite-Blocklength and Error-Exponent Analyses for LDPC Codes in Point-to-Point and Multiple Access Communication
}

\author{
Yuxin Liu and Michelle Effros \\ Department of Electrical Engineering, California Institute of Technology, Pasadena 91125, USA. \\ Email:\{yuxinl, effros\}@caltech.edu
}

\begin{abstract}
This paper applies error-exponent and dispersionstyle analyses to derive finite-blocklength achievability bounds for low-density parity-check (LDPC) codes over the point-to-point channel (PPC) and multiple access channel (MAC). The errorexponent analysis applies Gallager's error exponent to bound achievable symmetrical and asymmetrical rates in the MAC. The dispersion-style analysis begins with a generalization of the random coding union (RCU) bound from random code ensembles with i.i.d. codewords to random code ensembles in which codewords may be statistically dependent; this generalization is useful since the codewords of random linear codes such as random LDPC codes are dependent. Application of the RCU bound yields improved finite-blocklength error bounds and asymptotic achievability results for i.i.d. random codes and new finiteblocklength error bounds and achievability results for LDPC codes. For discrete, memoryless channels, these results show that LDPC codes achieve first- and second-order performance that is optimal for the PPC and identical to the best-prior results for the MAC.
\end{abstract}

\section{INTRODUCTION}

$\mathbf{L}$ OW-density parity-check (LDPC) codes are linear codes designed with sparse parity-check matrices for the purpose of enabling low complexity decoding strategies. Introduced along with corresponding iterative decoding algorithms by Gallager in 1962 [1] and largely overlooked until their rediscovery with the introduction of turbo codes [2] in the 1990 s, LDPC codes are now in widespread use, playing a role in commercial standards like $10 \mathrm{~Gb} / \mathrm{s}$ Ethernet (IEEE 803.3an), WiFi (IEEE 802.11n), WiMAX (IEEE 802.16e), and the 5G standard [3].

This paper presents achievability bounds for the finiteblocklength performance of LDPC codes over the point-topoint channel (PPC) and the multiple access channel (MAC). Proofs employ two types of analyses.

1) Error-exponent analyses generalize the techniques in [4] to demonstrate that average error probability $\epsilon$ decays exponentially in blocklength $n$ with an error exponent bounded below by Gallager's error exponent. This technique yields tighter bounds when $\epsilon$ is very small.

2) Dispersion-style analyses generalize [5], bounding the log size of the codebook achievable for a given average

This material is based upon work supported by the National Science Foundation under Grant No. 1817241. The work of Y. Liu is supported in part by the Oringer Fellowship Fund in Information Science and Technology.
TABLE I

SUMMARY OF NOTATIONS

\begin{tabular}{|c|c|}
\hline$n$ & blocklength/number of LDPC variable nodes \\
\hline$r$ & number of LDPC check nodes \\
\hline$\lambda$ & variable node degree of regular LDPC code \\
\hline$\rho$ & check node degree of regular LDPC code \\
\hline$c$ & single-transmitter codebook \\
\hline$d$ & MAC codebook \\
\hline $\mathcal{Q}$ & $\mathrm{GF}(q)^{K}$ \\
\hline $\mathcal{G}$ & bipartite LDPC graph \\
\hline $\mathcal{V}$ & vertex set of a graph $\mathcal{G}$ \\
\hline $\mathcal{E}$ & edge set of a graph $\mathcal{G}$ \\
\hline$i(x ; y)$ & information density \\
\hline$C$ & channel capacity \\
\hline$V$ & channel dispersion \\
\hline$T$ & third-order centered moment of information density \\
\hline$Q$ & complementary Gaussian CDF \\
\hline $\mathcal{T}_{q}^{n}$ & set of all possible types for $n$ elements from $\mathrm{GF}(q)$ \\
\hline $\mathcal{T}_{\mathcal{O}}^{n}$ & set of all possible types for $n$ elements from $\mathcal{Q}$ \\
\hline$v$ & LDPC coset vector \\
\hline$\delta$ & LDPC quantizer \\
\hline $\bar{S}^{n}(\boldsymbol{t})$ & ensemble-average number of type- $\boldsymbol{t}$ codewords/codematrices \\
\hline$\overline{\boldsymbol{S}}^{n}$ & ensemble-average spectrum \\
\hline $\mathcal{D}(g)$ & Bhattacharyya parameter for input $g$ \\
\hline$B(n, \boldsymbol{t})$ & multinomial coefficient \\
\hline$E_{p}(R)$ & Gallager's error exponent for distribution $p$ \\
\hline
\end{tabular}

error probability $\epsilon$ and blocklength $n$. This method yields tighter bounds when $n$ is very small.

We begin with a brief overview of prior LDPC and linear coding analyses.

In his 1968 text [6, Section 6.2], Gallager describes a random coset parity-check matrix code ensemble. Each element of the parity-check matrix is chosen uniformly and independently from $\{0,1\}$. The coset ensemble is formed by adding the same random vector to all codewords defined by the parity-check matrix. For PPCs with non-binary input alphabets, a "quantization" mapping maps one or more binary vectors to each channel input symbol. Gallager shows that the proposed code can achieve the capacity of an arbitrary discrete, memoryless PPC (DM-PPC) under maximum likelihood (ML) decoding.

In [7], Davey and MacKay generalize binary LDPC codes to finite field $\operatorname{GF}(q), q \geq 2$, showing empirically that $q$-ary codes can significantly improve binary code performance for binary-input PPCs under belief propagation decoding.

The first analysis of the standard $\operatorname{GF}(q)$ LDPC code 
ensemble appears in [4]. The standard GF $(q)$ LDPC code ensemble employs a random Tanner graph that maps the vector of variable-node edge sockets to a random permutation of the vector of check-node edge sockets; edge weights are independent and identically distributed (i.i.d.) uniformly on $\operatorname{GF}(q) \backslash\{0\}$. For the DM-PPC under ML decoding, [4] derives an upper bound on the average error probability using Gallager's error exponent, showing that the random code has a high probability under sufficiently large connectivity and blocklength of achieving vanishing error probability at rates arbitrarily close to the channel capacity. Independently of [4], the authors in [8] analyze the performance over moduloadditive PPCs of two different GF $(q)$-LDPC code ensembles under ML decoding. The error exponents for most codes in their design are bounded below asymptotically by the random coding error exponent [8].

While the above studies focus on asymptotic behavior of LDPC code ensembles, the increasing prevalence of delay sensitive applications motivate finite-blocklength (nonasymptotic) code analyses. For example, blocklengths of current 5G LDPC and polar codes typically range from 100 to 20000

In [9], Di et al. analyze the finite-blocklength performance of LDPC codes over the binary erasure channel (BEC), where finite-blocklength analysis boils down to a combinatorial problem. The paper derives the exact average bit- and block-erasure probability for a given regular ensemble of LDPC codes under an iterative decoding algorithm and presents upper bounds on the average bit- and block-erasure probability for standard binary LDPC code ensembles and the random parity-check ensemble under ML decoding. Other studies that focus on the BEC include [10]-[12]. The work in [13], [14] extends the finite-blocklength analysis to general (not necessarily symmetric) binary-input channels.

Unfortunately, the above-described non-asymptotic analyses yield expressions that are either difficult to evaluate or depend on empirical performance. As a result, they provide less insight than the dispersion-style bounds (with corresponding converse results) found in [5], which accurately characterize the backoff from channel capacity using the channel dispersion $V$ and target error $\epsilon$ for blocklengths as short as 100. This observation motivates our generalization of the dispersion-style analyses to the standard LDPC code ensemble.

Yang and Meng [15] study Gallager's independent, uniform parity-check ensemble and the standard binary LDPC code ensemble under modified Feinstein's threshold decoding. Noting that codewords under these ensembles are not pairwise independent and therefore that Shannon-style random coding arguments do not apply, they derive new achievability bounds for memoryless binary-input output-symmetric PPCs, demonstrating that Gallager's parity-check ensemble bound is asymptotically tight up to the second order and that the standard LDPC code ensemble is capacity achieving.

Fewer analyses are available for LDPC codes over MACs. In [16] and [17], the authors study the two-user Gaussian MAC with BPSK modulation using LDPC codes. The main results in [16] are two different approximations for the density evolution, which lead to a simple linear programming optimization for MAC LDPC code design. The authors of [17] adopt a belief propagation (BP) algorithm, and derive the probability density function (PDF) of the log-likelihood-ratios (LLRs) fed to the component LDPC decoders. The authors of [18] consider LDPC coset codes in a compound MAC with common information and analyze the performance of the proposed coset codes by deriving a lower bound on error exponents. In [19], Ebrahimi et al. introduce a two-layer coded channel access framework and analyze its performance over erasure adder MACs and a random access network where the number of active users is known at the receiver. The paper presents density evolution analysis in cases where the outer layer is a long-blocklength LDPC code.

The finite-blocklength performance of the standard LDPC code ensemble under either an arbitrary DM-PPC or discrete, memoryless MAC (DM-MAC) remains an open problem.

This paper analyzes the finite-blocklength performance of the standard GF $(q)$ LDPC code ensemble under ML decoding using both the error-exponent approach from [4] and dispersion-style approach from [5].

For the error-exponent analysis, we extend the result of [4] from the DM-PPC to symmetrical rates in the $K$-transmitter DM-MAC (DM- $K$-MAC) and arbitrary rates in the DM-2MAC using Gallager's error exponent; the latter generalizes to $K$-transmitter MACs for $K>2$. We then refine the result by providing a non-asymptotic expansion of Gallager's error exponent using [6, Exercise 5.23].

For the dispersion-style approach, we derive finiteblocklength error bounds and asymptotic third-order achievability results for the DM-PPC and the DM-2-MAC for i.i.d. codes; the achievability result is optimal up to the third order in the DM-PPC case, improving the corresponding bound on the number of codewords achievable under a desired error probability bound from a third-order term $O(\log n)$ in 5 Th. 49] to $\frac{1}{2} \log n-O(1)$ and matching the corresponding converse bound [5. Th. 48] up to the third order. For the DM-2MAC, our bound improves the third-order MAC achievability bound from $-\nu \log n \mathbf{1}$ with $\nu \geq 2\left|\mathcal{X}_{1}\right|\left|\mathcal{X}_{2}\right||\mathcal{Y}|$ in [20] to $\frac{1}{2} \log n \mathbf{1}-O(1) \mathbf{1}$. As noted in [15], random LDPC code are random linear codes, and the use of an underlying paritycheck matrix results in statistically dependent codewords. We therefore need to generalize the random coding union (RCU) bound [5, Th. 16] from codes employing i.i.d. codeword design to a more general family of randomly designed codes that includes codes with statistically dependent codewords. We use our generalized RCU bound to derive an upper bound for the standard LDPC code ensemble with coset vector and quantization, showing that LDPC codes achieve first- and second-order performance that is optimal for the DM-PPC and identical to the best-prior results for the DM-MAC.

Remark 1: Although practical implementations of LDPC codes typically employ fast but sub-optimal decoders, it is instructive to study the performance of LDPC codes under ML decoding in order to distinguish how much performance penalty, if any, results from the application of a low density encoder and separate this impact from the impact of suboptimal decoding.

The organization of this paper is as follows. Section [II-A 
defines notation. Section $\amalg$ II-B introduces our channel models. Section II-C defines the quantized coset LDPC codes used in our study. Sections III-A and III-B apply the error-exponent approach to bound the performance of quantized coset LDPC codes with ML decoding on the DM-MAC; the analysis treats both communication at a symmetrical rate point in an arbitrary symmetrical DM- $K$-MAC, and communication at an asymmetrical rate point for an arbitrary DM-2-MAC. Section III-C relates the error exponent results to the dispersion-style results, revealing that the error-exponent analysis achieves a sub-optimal second-order coefficient in blocklength $n$ but a superior bound when target error probability $\epsilon$ is small. Sections IV-A and IV-B present the performance of standard i.i.d. codes for the DM-PPC and DM-MAC using the RCU bound; the resulting bounds are optimal to the third-order for the DM-PPC and the tightest result to date for the DMMAC. In Section V-A we apply the generalized RCU bound to quantized coset LDPC codes, which lack the property of codeword independence used in bounding code performance in the DM-PPC. We present both a finite-blocklength error bound and an asymptotic achievability result that is optimal up to the second order. Section $\mathrm{V}-\mathrm{B}$ extends the result to the DM2-MAC, showing that LDPC codes achieve first- and secondorder performance that is identical to the best-prior results for the DM-2-MAC.

The main results of this paper are Theorems 1, 2, and 4 which bound the error exponent performance of the quantized coset LDPC code; Theorems 11 and 14, which give the finiteblocklength error bound and asymptotic achievability result for standard i.i.d. codes; and Theorems 15 and 16 , which present a finite-blocklength error bound and asymptotic achievability result for the quantized coset LDPC code.

\section{Definitions AND Notation}

\section{A. Notation}

Throughout this paper, we denote the set of integers $\left\{1,2, \ldots, k_{1}\right\}$ as $\left[k_{1}\right]$, and $\left\{k_{1}, \ldots, k_{2}\right\}$ as $\left[k_{1}: k_{2}\right]$ for any positive integers $k_{1}$ and $k_{2}$, where $\left[k_{1}: k_{2}\right]=\emptyset$ when $k_{1}>k_{2}$. We use uppercase letters (e.g., $X$ and $Y$ ) for random variables, lowercase letters (e.g., $x$ and $y$ ) for realizations of the corresponding random variables, and calligraphic uppercase letters (e.g., $\mathcal{X}$ and $\mathcal{Y}$ ) for sample spaces. To represent vectors, we use both superscripts (e.g., $x^{n}$ and $X^{n}$ ) and bold face (e.g., $\mathbf{x}$ and $\mathbf{1}=(1, \ldots, 1))$ when the length of the vector is clear from the context. We use both $X_{i}$ and $\boldsymbol{X}[i]$ to represent the $i$ th element of the vector $\boldsymbol{X}=X^{n}$. For any scalar function $f(\cdot)$ and any vector $\mathbf{x} \in \mathbb{R}^{n}, f(\mathbf{x})$ is the vector of function values, defined as $f(\mathbf{x}) \triangleq\left(f\left(x_{i}\right), i \in[n]\right)$. Given a set $\mathcal{Z} \subseteq \mathbb{R}^{n}$, a vector $\boldsymbol{v} \in \mathbb{R}^{n}$, and a scalar $a \in \mathbb{R}, a \mathcal{Z}+\boldsymbol{v} \triangleq\{a \boldsymbol{z}+\boldsymbol{v}, \boldsymbol{z} \in \mathcal{Z}\}$.

For any joint distribution $P_{X Y}$ on discrete alphabet $\mathcal{X} \times \mathcal{Y}$, we denote the information density by

$$
i(x ; y) \triangleq \log \frac{P_{X Y}(x, y)}{P_{X}(x) P_{Y}(y)}=\log \frac{P_{Y \mid X}(y \mid x)}{P_{Y}(y)} .
$$

Given a set $\mathcal{X}$, we denote the $n$-fold Cartesian product of $\mathcal{X}$ as $\mathcal{X}^{n}$ and indicate a probability distribution on $\mathcal{X}^{n}$ by $P_{X^{n}}$. For any alphabets $\mathcal{X}_{i}, i \in[n]$ and any countable ordered set
$\mathcal{A} \subseteq[n]$, we define $\mathcal{X}_{\mathcal{A}} \triangleq \prod_{i \in \mathcal{A}} \mathcal{X}_{i}$ and let $P_{X_{\mathcal{A}}}$ denote a distribution on the alphabet $\mathcal{X}_{\mathcal{A}}$. We say $x_{\mathcal{A}} \geq y_{\mathcal{A}}$ if $x_{a} \geq y_{a}$ for all $a \in \mathcal{A}$. For any joint distribution $P_{X^{n} Y}$ on $\mathcal{X}^{n}, \mathcal{Y}$ and any ordered sets $\mathcal{A}$ and $\mathcal{B}$ with $\mathcal{A} \cap \mathcal{B}=\emptyset$, and any $x_{\mathcal{A}} \in \mathcal{X}_{\mathcal{A}}, x_{\mathcal{B}} \in \mathcal{X}_{\mathcal{B}}$, and $y \in \mathcal{Y}$

$$
\begin{aligned}
i\left(x_{\mathcal{A}} ; y\right) & \triangleq \log \frac{P_{Y \mid X_{\mathcal{A}}}\left(y \mid x_{\mathcal{A}}\right)}{P_{Y}(y)} \\
i\left(x_{\mathcal{A}} ; y \mid x_{\mathcal{B}}\right) & \triangleq \log \frac{P_{Y_{X_{\mathcal{A}}, X_{\mathcal{B}}}\left(y \mid x_{\mathcal{A}}, x_{\mathcal{B}}\right)}}{P_{Y \mid X_{\mathcal{B}}}\left(y \mid x_{\mathcal{B}}\right)} .
\end{aligned}
$$

The mutual informations, dispersions, conditioned dispersions, and third centered moments of information are

$$
\begin{aligned}
I\left(P_{X_{\mathcal{A}}}\right) & \triangleq \mathbb{E}\left[i\left(X_{\mathcal{A}} ; Y\right)\right] \\
I\left(P_{X_{\mathcal{A}}} \mid P_{X_{\mathcal{B}}}\right) & \left.\triangleq \mathbb{E}\left[i\left(X_{\mathcal{A}} ; Y \mid X_{\mathcal{B}}\right)\right)\right] \\
V\left(P_{X_{\mathcal{A}}}\right) & \triangleq \operatorname{Var}\left[i\left(X_{\mathcal{A}} ; Y\right)\right] \\
V\left(P_{X_{\mathcal{A}}} \mid P_{X_{\mathcal{B}}}\right) & \triangleq \operatorname{Var}\left[i\left(X_{\mathcal{A}} ; Y \mid X_{\mathcal{B}}\right)\right] \\
V^{Y}\left(P_{X_{\mathcal{A}}}\right) & \triangleq \operatorname{Var}\left[i\left(X_{\mathcal{A}} ; Y\right) \mid Y\right] \\
V^{Y}\left(P_{X_{\mathcal{A}}} \mid P_{X_{\mathcal{B}}}\right) & \triangleq \operatorname{Var}\left[i\left(X_{\mathcal{A}} ; Y \mid X_{\mathcal{B}}\right) \mid Y\right] \\
T_{(}\left(P_{X_{\mathcal{A}}}\right) & \triangleq \mathbb{E}\left[\left|i\left(X_{\mathcal{A}} ; Y\right)-I\left(P_{X_{\mathcal{A}}}\right)\right|^{3}\right] \\
T\left(P_{X_{\mathcal{A}}} \mid P_{X_{\mathcal{B}}}\right) & \left.\left.\triangleq \mathbb{E}\left[\mid i\left(X_{\mathcal{A}} ; Y \mid X_{\mathcal{B}}\right)-I\left(P_{X_{\mathcal{A}}} \mid P_{X_{\mathcal{B}}}\right)\right]\right|^{3}\right] .
\end{aligned}
$$

The cumulative distribution function $(\mathrm{CDF})$ and PDF for standard Gaussian distribution $\mathcal{N}(0,1)$ are denoted by

$$
\begin{aligned}
& \Phi(x) \triangleq \frac{1}{\sqrt{2 \pi}} \int_{-\infty}^{x} e^{-\frac{u^{2}}{2}} d u, \\
& \phi(x) \triangleq \frac{1}{\sqrt{2 \pi}} e^{-\frac{x^{2}}{2}},
\end{aligned}
$$

respectively. The function $Q(\cdot)$ denotes the standard Gaussian complementary CDF

$$
Q(x) \triangleq 1-\Phi(x)=\frac{1}{\sqrt{2 \pi}} \int_{x}^{\infty} e^{-\frac{u^{2}}{2}} d u,
$$

and $Q^{-1}(\cdot)$ is the inverse function of $Q(\cdot)$.

We use $P_{X(1) \ldots X(M)}$ to denote the distribution of a codebook with $M$ codewords. For any ordered set $\mathcal{A} \subseteq[M]$, the notation $X(\mathcal{A})=(X(i), i \in \mathcal{A})$ captures a subset of the codewords.

Throughout this paper, the base of all logarithms and exponentials, unless otherwise indicated, is $q$, where prime power $q$ specifies the alphabet for the $\operatorname{GF}(q)$-LDPC code defined in the next section. We employ standard $o(\cdot)$ and $O(\cdot)$ notations writing $f(n)=o(g(n))$ if $\lim _{n \rightarrow \infty}\left|\frac{f(n)}{g(n)}\right|=0$ and $f(n)=O(g(n))$ if there exist constants $a$ and $n_{0}$ such that $|f(n)| \leq a|g(n)|$ for all $n>n_{0}$.

\section{B. Channel Models: DM-PPC and DM-MAC}

Definition 1: (DM-PPC) A DM-PPC is described by

$$
\left(\mathcal{X}, P_{Y \mid X}, \mathcal{Y}\right)
$$

where $\mathcal{X}$ and $\mathcal{Y}$ are the discrete channel input and output alphabets, respectively, and $P_{Y \mid X}(y \mid x)$ specifies the channel 
transition probability for all $x \in \mathcal{X}$ and $y \in \mathcal{Y}$. The $n$-th order extension $\left(\mathcal{X}^{n}, P_{Y^{n} \mid X^{n}}, \mathcal{Y}^{n}\right)$ of $\left(\mathcal{X}, P_{Y \mid X}, \mathcal{Y}\right)$ satisfies $\operatorname{Pr}\left[y_{k} \mid x^{k}, y^{k-1}\right]=\operatorname{Pr}\left[y_{k} \mid x_{k}\right]$ for all $k \in[n]$.

Definition 2: (DM- $K$-MAC) A DM- $K$-MAC is defined by

$$
\left(\prod_{i=1}^{K} \mathcal{X}_{i}, P_{Y \mid \boldsymbol{X}}, \mathcal{Y}\right)
$$

where $\mathcal{X}_{i}, i \in[K]$, and $\mathcal{Y}$ are the discrete channel input and output alphabets, respectively, and $P_{Y \mid X}=P_{Y \mid X_{1}, X_{2}, \ldots, X_{K}}$ is the channel transition probability. A DM- $K$-MAC is called symmetric if all transmitters have the same input alphabet $\mathcal{X}_{i}=\mathcal{X}$ for all $i \in[K]$ and

$$
P_{Y \mid \boldsymbol{X}}(y \mid \boldsymbol{x})=P_{Y \mid \boldsymbol{X}}(y \mid \pi(\boldsymbol{x}))
$$

for all $y \in \mathcal{Y}, \boldsymbol{x} \in \mathcal{X}^{K}$, and permutations $\pi$ on $[K]$.

\section{Quantized Coset Codes}

We begin with a formal definition of the quantized coset GF $(q)$-LDPC code used in our study.

For any prime power $q$ and finite field $\operatorname{GF}(q)$, a quantized coset $\operatorname{GF}(q)$-LDPC code is defined by three components: a standard LDPC encoder, a coset vector $\boldsymbol{v}$, and a quantizer $\delta$, defined below and illustrated in Figure 1 .



Fig. 1. Encoding of Quantized Coset LDPC Code

Definition 3: (Standard GF $(q)$-LDPC code) A standard GF $(q)$-LDPC code is defined using a bipartite Tanner graph $\mathcal{G}=(\mathcal{V}, \mathcal{E})$ with $n$ variable nodes, $r$ check nodes, and edge set $\mathcal{E} \subseteq[n] \times[r]$. For each $(i, j) \in \mathcal{E},(i, j)$ represents an undirected edge connecting the $i$ th variable node and the $j$ th check node; each edge $(i, j) \in \mathcal{E}$ carries a constant $g_{i, j} \in \mathrm{GF}(q) \backslash\{0\}$. The notation

$$
\mathcal{N}(j) \triangleq\{i:(i, j) \in \mathcal{E}\},
$$

captures the neighborhood of check node $j \in[r]$ resulting from edge set $\mathcal{E}$.

The $n$ variable nodes hold a column vector $\boldsymbol{u}$ from $\operatorname{GF}(q)^{n}$. Vector $\boldsymbol{u}$ is a codeword if it satisfies all check nodes, giving

$$
\sum_{i \in \mathcal{N}(j)} g_{i, j} u_{i}=0 \quad \forall j \in[r] ;
$$

the linear equation operates in $\operatorname{GF}(q)$. The set of all $M=|\boldsymbol{c}|$ codewords constitute the codebook

$$
\boldsymbol{c}=\left\{\boldsymbol{c}_{1}, \ldots, \boldsymbol{c}_{M}\right\} \subseteq \mathrm{GF}(q)^{n}
$$

for the given Tanner graph $\mathcal{G}=(\mathcal{V}, \mathcal{E})$.

Following [21], [22], we do not transmit codewords from the LDPC encoder but instead apply quantized coset coding.

Definition 4: (Coset GF $(q)$-LDPC Code) Given a Tanner graph $\mathcal{G}=(\mathcal{V}, \mathcal{E})$ and the corresponding LDPC codebook $\boldsymbol{c}$, we obtain the coset LDPC code by adding a constant vector $\boldsymbol{v}$, called the coset vector, to each codeword $\boldsymbol{c}_{i} \in \boldsymbol{c}$. The addition

$$
\boldsymbol{c}_{i}+\boldsymbol{v}, i \in[M]
$$

is performed component-wise in $\operatorname{GF}(q)$. The set $\left\{\boldsymbol{c}_{i}+\boldsymbol{v}, i \in\right.$ $[M]\}$ is the codebook for the coset GF $(q)$-LDPC code.

Definition 5: (Quantized Coset GF $(q)$-LDPC Code) Given an LDPC codebook $\boldsymbol{c}$ and a coset vector $\boldsymbol{v}$, we map each symbol from $c_{i}+\boldsymbol{v}$ to a symbol from the channel input alphabet $\mathcal{U}$ using quantizer $\delta$ :

$$
\delta: \operatorname{GF}(q) \rightarrow \mathcal{U} .
$$

Mapping $\delta$ is applied component-wise; we therefore employ notation

$$
\delta\left(\boldsymbol{c}_{i}+\boldsymbol{v}\right) \triangleq\left[\delta\left(\boldsymbol{c}_{i}[j]+\boldsymbol{v}[j]\right)\right]_{j \in[n]}=\left[\delta\left(\left(\boldsymbol{c}_{i}+\boldsymbol{v}\right)[j]\right)\right]_{j \in[n]}
$$

for coset codeword $\boldsymbol{c}_{i}+\boldsymbol{v}$. The set $\left\{\delta\left(\boldsymbol{c}_{i}+\boldsymbol{v}\right), i \in[M]\right\}$ is the codebook for the quantized coset GF $(q)$-LDPC code.

The quantizer $\delta$ enables us to approximate, using a code on $\mathrm{GF}(q)$, any rational probability mass function $P_{U}$, for which $P_{U}(u)$ is an integer multiple $N_{u}$ of $1 / q$ for every $u \in \mathcal{U}$ (giving $P_{U}(u)=N_{u} / q$ ). This is achieved by mapping $N_{u}$ elements to each channel input symbol $u \in \mathcal{U}$.

Remark 2: The quantization $\delta(\cdot)$ is an essential component in code designs for arbitrary (not necessarily symmetric) DMPPCs since unequal channel transition probabilities between input and output symbols can lead to non-uniform capacityachieving input distributions. The performance penalty for using a uniform input distribution in place of the optimal input distribution is called the shaping gap.

Our analysis focuses on a random ensemble of quantized coset $\mathrm{GF}(q)$-LDPC codes.

We restrict attention to regular Tanner graphs, in which all left nodes have degree $\lambda$ and all right nodes have degree $\rho$. A random graph is chosen by first labeling the $|\mathcal{E}|$ edge sockets from left nodes from 1 to $|\mathcal{E}|$, then labeling the $|\mathcal{E}|$ edge sockets from right nodes from 1 to $|\mathcal{E}|$, and finally choosing a permutation $\pi$ uniformly at random from the set of permutations on $[|\mathcal{E}|]$. The graph connects each left node edge socket $i$ to the right-node edge socket $\pi_{i}$. The edge constant $g_{i, j}$ for each edge $(i, j) \in \mathcal{E}$ is chosen uniformly and independently at random from $\operatorname{GF}(q) \backslash\{0\}$.

Remark 3: An attracting property of regular LDPC codes is that the minimum distance grows linearly with blocklength [1], therefore regular LDPC codes achieve superior performance than irregular LDPC codes under ML decoding. In contrast, lower iterative decoding threshold makes irregular LDPC codes outperform regular LDPC codes under iterative decoding [23].

The design rate of the described ensemble is $R q$-ary symbols per channel use, where

$$
R \triangleq 1-\frac{r}{n}=1-\frac{\lambda}{\rho} .
$$

The actual number of legitimate codewords is $q^{n R}$ if the parity-check matrix corresponding to the randomly drawn Tanner graph has full rank and larger if that parity-check matrix does not have full rank. We restrict the operational 
rate to equal the design rate by choosing exactly $q^{n R}$ active codewords for use in coding. Before communication begins, the codebook, coset vector, and quantizer are revealed to all parties, so that the receiver knows which $M=q^{n R}$ codewords are employed and how they are processed. We refer to the process of selecting precisely $q^{n R}$ codewords for active use and effectively removing others from the codebook as codeword removal.

Definition 6: (Codeword Removal) Given an ensemble of $\mathrm{GF}(q)$-LDPC codes with design rate $R$, the codeword removal process generates an ensemble by dividing the probability of each code in the original ensemble equally among all code(s) corresponding to a distinct combination of $q^{n R}$ codewords from the original code.

We denote the random ensemble of $\operatorname{GF}(q)$-LDPC codes resulting from random Tanner graph design by $\operatorname{LDPC}(\mathrm{Full}, \lambda, \rho ; n)$; the random ensemble of $\mathrm{GF}(q)$-LDPC codes - after codeword removal but before coset addition or application of quantizer $\delta$ - by $\operatorname{LDPC}(\lambda, \rho ; n)$; and the random ensemble of quantized coset $\operatorname{GF}(q)$-LDPC codes by $\operatorname{LDPC}(\lambda, \rho, \delta ; n)$.

\section{ERROR-EXPONENT BOUNDS FOR LDPC CODE ENSEMBLE ON MAC}

A. Error-Exponent Bound for LDPC Code Ensemble on the DM-K-MAC with Identical Encoders

In this section, we consider an arbitrary, symmetric DM- $K$ MAC and derive the expected ensemble error probability under ML decoding. In this analysis, we assume that all transmitters employ the same random codebook from the $\operatorname{LDPC}(\lambda, \rho ; n)$ ensemble, but each is offset by an independent random coset vector $\boldsymbol{v}_{j}, j \in[K]$. All transmitters employ the same quantizer $\delta(\cdot)$.

For a fixed LDPC graph with $M=q^{n R}$ codewords $\boldsymbol{c}_{1}, \ldots, \boldsymbol{c}_{M} \in \mathrm{GF}(q)^{n}$, the single-transmitter codebook for transmitter $k$ is

$$
\boldsymbol{c}_{(k)}=\left\{\boldsymbol{c}_{1}, \ldots, \boldsymbol{c}_{M}\right\} \subseteq \mathrm{GF}(q)^{n}
$$

for each $k \in[K]$. The MAC codebook is the set of codematrices

$$
\boldsymbol{d}=\left\{\boldsymbol{d}_{\boldsymbol{m}}: \boldsymbol{m} \in[M]^{k}\right\} \subseteq \mathrm{GF}(q)^{n \times K}
$$

that result from those codewords, where for any $\boldsymbol{m}=$ $(m(1), \ldots, m(K)) \in[M]^{K}, \boldsymbol{d}_{\boldsymbol{m}}=\left(\boldsymbol{c}_{m(1)}, \ldots, \boldsymbol{c}_{m(K)}\right)$.

We denote the MAC ensemble before restriction of codematrices by $\operatorname{LDPC}_{K}($ Full, $\lambda, \rho ; n)$. After random selection of $q^{n R}$ codewords from which we build MAC codematrices, we denote the MAC ensemble before and after applying the random coset matrix and fixed quantization by $\operatorname{LDPC}_{K}(\lambda, \rho ; n)$ and $\operatorname{LDPC}_{K}(\lambda, \rho, \delta ; n)$, respectively.

Let $\boldsymbol{v}$ denote the coset matrix formed by combining the $K$ coset vectors column-wise, giving $\boldsymbol{v}=\left[\boldsymbol{v}_{1} \boldsymbol{v}_{2} \cdots \boldsymbol{v}_{K}\right]$. We map each symbol from the matrix $\boldsymbol{d}_{\boldsymbol{m}}+\boldsymbol{v} \in \mathrm{GF}(q)^{n \times K}$ to a symbol from the channel input alphabet $\mathcal{U}$ using the (component-wise) quantizer $\delta$. The resulting channel input is

$$
\delta\left(\boldsymbol{d}_{\boldsymbol{m}}+\boldsymbol{v}\right)
$$

Remark 4: As noted in [24], using the same codebook from the $\operatorname{LDPC}(\lambda, \rho ; n)$ ensemble for all transmitters has practical advantages. In our case, each device is the same except for its unique random coset vector $\boldsymbol{v}_{j}$. When considering an arbitrary (not necessarily symmetric) DM- $K$-MAC or an arbitrary rate vector, a different quantized coset LDPC code $\operatorname{LDPC}\left(\lambda_{j}, \rho_{j}, \delta_{j} ; n\right), j \in[K]$ can be applied to each transmitter. For simplicity of notation, we assume in this section that both the MAC and the desired rate are symmetric. General MACs and rate vectors are studied in Section III-B for the case of $K=2$.

In order to analyze the expected ensemble error probability for some fixed value $(\lambda, \rho)$, we require a means of describing the distribution over the types of codematrices. The following definitions are useful for that discussion.

For any matrix $\boldsymbol{a} \in \operatorname{GF}(q)^{n \times K}$, recall that $\mathcal{Q} \triangleq \operatorname{GF}(q)^{K}$ specifies the alphabet of each row of $\boldsymbol{a}$. Let $\mathcal{T}_{\mathcal{Q}}^{n}(\boldsymbol{a})$ denote the type that results when we view $\boldsymbol{a}$ as a list of $n$ elements from alphabet $\mathcal{Q}$, giving

$$
\begin{aligned}
\mathcal{T}_{\mathcal{Q}}^{n}(\boldsymbol{a}) & =(t(g): g \in \mathcal{Q}), \\
t(g) & =\sum_{i=1}^{n} 1(a[i, *]=g) .
\end{aligned}
$$

If $\boldsymbol{a}=\boldsymbol{d}_{\boldsymbol{m}}$ for some codematrix $\boldsymbol{d}_{\boldsymbol{m}}$, then $\mathcal{T}_{Q}^{n}(\boldsymbol{a})$ captures, for each $g \in \mathcal{Q}$, the number of time steps when the $(K$ dimensional) row of codematrix $\boldsymbol{d}_{\boldsymbol{m}}$ takes value $g$. The set of possible types is

$$
\mathcal{T}_{\mathcal{Q}}^{n} \triangleq\left\{\mathcal{T}_{\mathcal{Q}}^{n}(\boldsymbol{a}): \boldsymbol{a} \in \mathrm{GF}(q)^{n \times K}\right\} \subset \mathbb{Z}_{+}^{|\mathcal{Q}|} .
$$

For any MAC codebook $\boldsymbol{d}$, let

$$
\boldsymbol{S}_{\boldsymbol{d}}^{n}=\left(S_{\boldsymbol{d}}^{n}(\boldsymbol{t}): \boldsymbol{t} \in \mathcal{T}_{\mathcal{Q}}^{n}\right)
$$

represent the spectrum of codebook $\boldsymbol{d}$, where for any type $\boldsymbol{t} \in \mathcal{T}_{\mathcal{Q}}^{n}$,

$$
S_{\boldsymbol{d}}^{n}(\boldsymbol{t})=\sum_{\boldsymbol{m}} 1\left(\mathcal{T}_{\mathcal{Q}}^{n}\left(\boldsymbol{d}_{\boldsymbol{m}}\right)=\boldsymbol{t}\right)
$$

is the number of codematrices of type $t$ in MAC codebook $\boldsymbol{d}$. When the code is chosen at random (e.g., through random LDPC graph design and random codeword removal), we use

$$
\overline{\boldsymbol{S}}^{n} \triangleq E_{\boldsymbol{D}}\left[\boldsymbol{S}_{\boldsymbol{D}}^{n}\right]=\left(\bar{S}^{n}(\boldsymbol{t}): \boldsymbol{t} \in \mathcal{T}_{\mathcal{Q}}^{n}\right)
$$

to represent the ensemble-average spectrum of the random codebook $D$, where $E_{D}[\cdot]$ here captures the expectation with respect to the random choice of codebook $D$.

The following notation is used in the statement of Theorem 11. Given a discrete, memoryless $K$-transmitter MAC with input alphabet $\mathcal{X}=\mathcal{U}^{K}$, channel transition $P_{Y \mid X}$, and quantizer $\delta(\cdot)$, let $\mathcal{D}=(\mathcal{D}(g): g \in \mathcal{Q})$, where

$$
\mathcal{D}(g) \triangleq \frac{1}{q^{K}} \sum_{g^{\prime} \in \mathcal{Q}} \sum_{y} \sqrt{P_{Y \mid X}\left(y \mid \delta\left(g^{\prime}\right)\right) P_{Y \mid X}\left(y \mid \delta\left(g^{\prime}+g\right)\right)}
$$

is the extension of Bhattacharyya parameter to non-binary channels. 
For any type $t \in \mathcal{T}_{\mathcal{Q}}^{n}$, let $\mathcal{D}^{t}$ be the product of terms $\mathcal{D}(g)$ resulting from type $t$, giving

$$
\mathcal{D}^{t} \triangleq \prod_{g \in \mathcal{Q}} \mathcal{D}(g)^{t(g)}
$$

and let $B(n, \boldsymbol{t})$ denote the number of distinct matrices $\boldsymbol{a} \in$ $\operatorname{GF}(q)^{n \times K}$ of type $t$, which is the multinomial coefficient

$$
B(n, \boldsymbol{t}) \triangleq \frac{n !}{\prod_{g \in \mathcal{Q}} t_{g} !} .
$$

Theorem 1 derives an upper bound on the ensemble-average error probability for the LDPC code ensemble as a function of the product of Bhattacharyya parameter $\mathcal{D}^{t}$, ensemble-average number of codematrices $\bar{S}^{n}(\boldsymbol{t})$, and Gallager's error exponent $E_{p}(\cdot)$, defined below.

Theorem 1: Let $P_{Y \mid X}$ be the transition probability for a symmetric DM- $K$-MAC with input alphabet $\mathcal{X}=\mathcal{U}^{K}$ and output alphabet $\mathcal{Y}$. Let the MAC's maximal symmetrical rate vector be the $K$-vector $(C, \ldots, C)$, and fix any $\boldsymbol{R}=$ $(R, \ldots, R)$ with $R<C$. Let $P_{U}$ be a pmf on $\mathcal{U}$ for which $P_{U}(u)=N_{u} / q$ for some integer $N_{u}$ for each $u \in \mathcal{U}$, and let $\delta: \operatorname{GF}(q) \rightarrow \mathcal{U}$ be a quantization matched to $P_{U}$. Consider any ensemble of random $K$-MAC LDPC codes, denoted by $\mathcal{L}$, with codeword removal and blocklength $n$, symmetrical rate $\boldsymbol{R}$, and ensemble-average spectrum $\overline{\boldsymbol{S}}^{n}$.

Let $\mathrm{T} \subseteq \mathcal{T}_{\mathcal{Q}}^{n}$ be any fixed set of types. Then for any blocklength $n$, the ensemble-average error probability of the quantized coset-shifted ensemble of $\mathcal{L}$ under ML decoding is bounded as

$$
E\left[P_{e}^{(n)}\right] \leq \sum_{\boldsymbol{t} \in \mathrm{T}} \bar{S}^{n}(\boldsymbol{t}) \mathcal{D}^{\boldsymbol{t}}+q^{-n E_{p}\left(K R+\left(\log \alpha_{\mathrm{MAC}}\right) / n\right)},
$$

where $E_{p}(\cdot)$ is Gallager's error exponent for the input distribution $P_{X}=P_{U^{K}}=P_{U}^{K}$, defined using

$$
\begin{aligned}
E_{p}(R) & \triangleq \max _{0 \leq \rho \leq 1}\left[E_{0}\left(\rho, P_{X}\right)-\rho R\right], \\
E_{0}\left(\rho, P_{X}\right) & \triangleq-\log \sum_{y}\left[\sum_{x \in \mathcal{U}^{K}} P_{X}(x) P_{Y \mid X}(y \mid x)^{1 /(1+\rho)}\right]^{1+\rho},
\end{aligned}
$$

and

$$
\alpha_{\mathrm{MAC}}=\max _{\boldsymbol{t} \in \mathrm{T}^{c}} \frac{\bar{S}^{n}(\boldsymbol{t})}{\left(M^{K}-1\right) B(n, \boldsymbol{t}) q^{-n K}} .
$$

Here $\mathrm{T}^{c}=\mathcal{T}_{\mathcal{Q}}^{n} \backslash \mathrm{T} \backslash\left\{\mathcal{T}_{\mathcal{Q}}^{n}(\mathbf{0})\right\}$, where $\mathcal{T}_{\mathcal{Q}}^{n}(\mathbf{0})$ is the type of the all zero codematrix, and $M=q^{n R}$.

Proof: See Appendix A

Remark 5: The tightest bound for each blocklength $n$ in Theorem 1 can be obtained by optimizing over the set of types $\mathrm{T}$.

Remark 6: The error probability expression in Theorem 1 takes the same form for different ensembles of $K$-MAC LDPC codes, but the ensemble-average spectrum, $\overline{\boldsymbol{S}}^{n}$, and consequently $\frac{\log \alpha_{\text {MAC }}}{n}$ vary for different ensembles.

Theorem 1 captures the error bound in two terms. In Theorem 2 below, we demonstrate that the first term in Theorem 1 can be made equal to zero for some non-trivial choice of $T$ provided that we first expurgate (remove) codes with small minimum distance. The definition of our expurgated code ensemble follows.

Definition 7: $\left(\operatorname{LDPC}_{K}-\operatorname{Ex}_{\sigma}(\lambda, \rho, \delta ; n)\right.$ ensemble) Let $P_{L}(\boldsymbol{D})$ denote the probability of observing a randomly chosen code $\boldsymbol{D}$ from the $\mathrm{LDPC}_{K}($ Full, $\lambda, \rho ; n)$ ensemble. The expurgated MAC LDPC code ensemble $\operatorname{LDPC}_{K}-\mathrm{Ex}_{\sigma}(\mathrm{Full}, \lambda, \rho ; n)$ is the ensemble obtained by placing probability zero on all codes of minimum distance less than or equal to $\sigma n$, and probability $\operatorname{Pr}_{L}\left(\boldsymbol{D} \mid d_{\min }(\boldsymbol{D})>\sigma n\right)$ on the remaining codes, giving

$$
\operatorname{Pr}_{\mathrm{ex}, \sigma}(\boldsymbol{D})= \begin{cases}0, & \text { if } d_{\min }(\boldsymbol{D}) \leq \sigma \\ \operatorname{Pr}_{L}\left(\boldsymbol{D} \mid d_{\min }(\boldsymbol{D})>\sigma n\right), & \text { otherwise. }\end{cases}
$$

Here the distance between two $n \times K$ codematrices $\boldsymbol{d}_{1}$ and $\boldsymbol{d}_{2}$, denoted by $d\left(\boldsymbol{d}_{1}, \boldsymbol{d}_{2}\right)$, is the number of rows that differ,

$$
d\left(\boldsymbol{d}_{1}, \boldsymbol{d}_{2}\right)=\sum_{i=1}^{n} \mathbb{1}\left(\boldsymbol{d}_{1}[i, *] \neq \boldsymbol{d}_{2}[i, *]\right)
$$

and the minimum distance of codebook $\boldsymbol{d}$ is

$$
d_{\min }(\boldsymbol{d})=\min _{\boldsymbol{m} \neq \boldsymbol{m}^{\prime}} d\left(\boldsymbol{d}_{\boldsymbol{m}}, \boldsymbol{d}_{\boldsymbol{m}^{\prime}}\right) .
$$

Applying the codeword removal process to the $\mathrm{LDPC}_{K^{-}}$ $\operatorname{Ex}_{\sigma}($ Full, $\lambda, \rho ; n)$ generates the $\operatorname{LDPC}_{K}-\operatorname{Ex}_{\sigma}(\lambda, \rho ; n)$ ensemble, and applying the coset addition and quantization to the $\mathrm{LDPC}_{K}-\mathrm{Ex}_{\sigma}(\lambda, \rho ; n)$ ensemble gives the the quantized coset MAC LDPC code ensemble $\operatorname{LDPC}_{K}-\operatorname{Ex}_{\sigma}(\lambda, \rho, \delta ; n)$.

For any $\lambda \geq 3$, the probability that an LDPC code drawn from the $\operatorname{LDPC}($ Full, $\lambda, \rho ; n)$ ensemble has a small minimum distance decays exponentially to zero as the blocklength $n$ grows [4, Th. 6]. In Appendix C, we show that the same bound applies after restriction to our fixed-rate code.

Since expurgation eliminates the first term in Theorem 1 the remainder of Theorem 2 works to demonstrate that the second term in Theorem 1 has the desired property.

In the DM-PPC, Gallager's error exponent has the property that $E_{p}(R)>0$ for all $R<C$. Here, similarly, $E_{p}(K R)>0$ for all $K R<K C$ in the DM- $K$-MAC (where $K R$ is the symmetrical sum-rate and $K C$ is the maximum symmetrical sum-rate). Notice, however, that the second term in Theorem 1 employs $E_{p}\left(K R+\frac{\log \alpha_{M A C}}{n}\right)$ rather than $E_{p}(K R)$. Theorem 2 therefore also seeks to evaluate the rate offset $\frac{\log \alpha_{\text {MAC }}}{n}$ in Gallager's error exponent. Using a series of supporting theorems provided in Appendix B. Theorem 2 shows that this rate offset can be made arbitrarily small. More precisely, Theorem 2 shows that if $\rho=\kappa n$ and $\kappa \rightarrow 0$ no more quickly than $\Theta\left(\frac{\log n}{n}\right)$, then $\frac{\log \alpha_{\text {MAC }}}{n}$ decays as $O\left(\frac{\log n}{n}\right)$. Therefore, our proposed code design is asymptotically capacity achieving.

Theorem 2: Let $P_{Y \mid X}$ be the transition probability for a discrete memoryless $K$-transmitter MAC with input alphabet $\mathcal{X}=\mathcal{U}^{K}$ and output alphabet $\mathcal{Y}$. Let the MAC's maximal symmetrical rate vector be the $K$-vector $(C, \ldots, C)$, and fix any $\boldsymbol{R}=(R, \ldots, R)$ with $R<C$. Let $P_{U}$ be a pmf on $\mathcal{U}$ for which $P_{U}(u)$ is an integer multiple of $1 / q$ for each $u \in \mathcal{U}$, and let $\delta: \operatorname{GF}(q) \rightarrow \mathcal{U}$ be a quantization matched to $P_{U}$. Let $\Delta R>0$ be some arbitrary number. Then for large enough $\rho$ and $n$, there exists LDPC parameters $(\lambda, \rho)$ 
for which the ensemble-average error probability for $\mathrm{LDPC}_{K}$ $\operatorname{Ex}_{\sigma}(\lambda, \rho, \delta ; n)$ ensemble under ML decoding is bounded as:

$$
E_{\mathrm{ex}}\left[P_{e}^{(n)}\right] \leq q^{-n E_{p}(K R+\Delta R)},
$$

where $E_{p}(\cdot)$ is Gallager's error exponent defined in Theorem 1 Further, if $\rho=\kappa n$ for some $\kappa$ that approaches zero no more quickly than $\Theta\left(\frac{\log n}{n}\right)$, then the minimum rate offset $\Delta R$ decays as $O\left(\frac{\log n}{n}\right)$.

Proof: See Appendix D.

Remark 7: Theorem 2 provides an upper bound on the ensemble-average error probability in terms of Gallager's error exponent $E_{p}(R)$ for input distribution $P_{X}=P_{U}^{K}$. Here $P_{U}$ is restricted to be a rational pmf for which, for all $u \in \mathcal{U}$, $P_{U}(u)=N_{u} / q$ for some integer $N_{u}$. By choosing $P_{U}$ to approximate the capacity-achieving input distribution, we obtain $E_{p}(R)>0$ for all $R<C$. Therefore, the ensemble-average error probability of $\mathrm{LDPC}_{K}-\operatorname{Ex}_{\sigma}(\lambda, \rho, \delta ; n)$ asymptotically approaches 0 , and the existence argument of a deterministic capacity-achieving quantized coset-shifted LDPC MAC code follows. However, note that the nature of the quantizer $\delta(\cdot)$ restrict achievable $P_{U}$ to be integer multiples of $\frac{1}{q}$. When the optimal input distribution $P_{X}^{*}$ is irrational or not an integer multiple of $\frac{1}{q}$, then a large alphabet size $q$ may be required to closely approximate $P_{X}^{*}$.

Our study chooses $M=q^{n R}$ codewords uniformly at random from the set of $q^{n R_{C}} \geq q^{n R}$ valid parity-check solutions. This approach differs from most other studies of LDPC codes, which assume that the parity-check matrix of a code randomly chosen from the $\operatorname{LDPC}(\lambda, \rho ; n)$ ensemble has full rank, giving $R_{C}=R$. This assumption is not precise, but it does become increasingly probable in the limit of large parity-check matrices. The following theorem formalizes this observation and demonstrates that the probability that the actual rate $R_{C}$ deviates from the design rate $R$ decays exponentially in the blocklength $n$.

Theorem 3: Consider the ensemble $\operatorname{LDPC}(\lambda, \rho ; n)$ without random codeword removal. Let $R \triangleq 1-\frac{\lambda}{\rho}$ denote the design rate of the ensemble and let $R_{C}$ denote the actual rate of a code $C$ from the ensemble using the full collection of legitimate codewords. For any $\epsilon>0$, there exists some integer $n(\epsilon)$ such that for $n>n(\epsilon)$

$$
\operatorname{Pr}\left[R_{\boldsymbol{C}}-R>\epsilon\right] \leq q^{-n \epsilon / 2} .
$$

In addition, for any $\epsilon>0$, there exists a $T_{0}>0$ such that for all $n>n(\epsilon)$

$$
\mathbb{E}\left[R_{C}-R\right] \leq T_{0} \frac{\log n}{n} .
$$

Proof: See Appendix E

\section{B. Error-Exponent Bound for LDPC Code Ensemble on the $D M-2-M A C$}

While the previous section treats the ensemble-average error probability for a symmetrical $K$-transmitter MAC with a symmetrical rate vector, this section gives the corresponding bound for a general 2-transmitter MAC with an arbitrary rate vector.
We first define the achievable rate region of a 2-transmitter MAC under a fixed input distribution. We then present the main error-exponent bound when LDPC code ensembles are employed.

Definition 8: Let $P_{Y \mid X_{1}, X_{2}}$ be the transition probability for an arbitrary DM-2-MAC. Let $\mathcal{R}\left(P_{X_{1}}, P_{X_{2}}\right)$ be the set of $\left(R_{1}, R_{2}\right)$ such that

$$
\begin{array}{r}
R_{1}<I\left(X_{1} ; Y \mid X_{2}\right) \\
R_{2}<I\left(X_{2} ; Y \mid X_{1}\right) \\
R_{1}+R_{2}<I\left(X_{1}, X_{2} ; Y\right),
\end{array}
$$

where the mutual informations are evaluated according to distribution $P_{Y \mid X_{1}, X_{2}} P_{X_{1}} P_{X_{2}}$.

Theorem 4: Let $P_{Y \mid X_{1}, X_{2}}$ be the transition probability for an arbitrary DM-2-MAC with input alphabet $\mathcal{X}=\mathcal{X}_{1} \times \mathcal{X}_{2}$ and output alphabet $\mathcal{Y}$. Let $P_{X_{i}}$ be a pmf on $\mathcal{X}_{i}$ for which $P_{X_{i}}\left(x_{i}\right)$ is an integer multiple of $1 / q$ for each $x_{i} \in \mathcal{X}_{i}$ and $i \in$ $\{1,2\}$. Let $\delta_{i}: \operatorname{GF}(q) \rightarrow \mathcal{X}_{i}$ be the corresponding quantization matched to $P_{X_{i}}, i \in\{1,2\}$. Assume transmitter $i$ employs a random code from the $\operatorname{LDPC}\left(\lambda_{i}, \rho_{i}, \delta_{i} ; n\right)$ ensemble $i \in$ $\{1,2\}$ with independent coset vector $\boldsymbol{v}_{i}$, such that the rate vector $\left(R_{1}, R_{2}\right) \in \mathcal{R}\left(P_{X_{1}}, P_{X_{2}}\right)$. Then for any blocklength $n$, the ensemble-average error probability under ML decoding is bounded as

$$
\begin{aligned}
E\left[P_{e}^{(n)}\right] \leq & q^{-n E_{p_{1}}\left(R_{1}+\frac{\log \alpha_{1}}{n}\right)}+q^{-n E_{p_{2}}\left(R_{2}+\frac{\log \alpha_{2}}{n}\right)} \\
& +q^{-n E_{p_{12}}\left(R_{1}+R_{2}+\frac{\log \alpha_{12}}{n}\right)}
\end{aligned}
$$

where $E_{p_{1}}(\cdot), E_{p_{2}}(\cdot)$ and $E_{p_{12}}(\cdot)$ are Gallager's error exponents for the input distributions $P_{X_{1}}, P_{X_{2}}$ and $P_{X}=P_{X_{1}} P_{X_{2}}$, defined using

$$
\begin{aligned}
& E_{p_{1}}(R) \triangleq \max _{0 \leq \rho \leq 1}\left[E_{0}^{1}\left(\rho, P_{X_{1}}\right)-\rho R\right] \\
& E_{p_{2}}(R) \triangleq \max _{0 \leq \rho \leq 1}\left[E_{0}^{2}\left(\rho, P_{X_{2}}\right)-\rho R\right] \\
& E_{p_{12}}(R) \triangleq \max _{0 \leq \rho \leq 1}\left[E_{0}^{12}\left(\rho, P_{X}\right)-\rho R\right] \\
& E_{0}^{1}\left(\rho, P_{X_{1}}\right) \triangleq-\log _{y} \sum_{x_{2} \in \mathcal{X}_{2}} P_{X_{2}}\left(x_{2}\right) \\
& \\
& {\left[\sum_{x_{1} \in \mathcal{X}_{1}} P_{X_{1}}\left(x_{1}\right) P_{Y \mid X_{1}, X_{2}}\left(y \mid x_{1}, x_{2}\right)^{\frac{1}{1+\rho}}\right]^{1+\rho}, } \\
& E_{0}^{2}\left(\rho, P_{X_{2}}\right) \triangleq-\log _{y} \sum_{x_{1} \in \mathcal{X}_{1}} P_{X_{1}}\left(x_{1}\right) \\
& {\left[\sum_{x_{2} \in \mathcal{X}_{2}} P_{X_{2}}\left(x_{2}\right) P_{Y \mid X_{1}, X_{2}}\left(y \mid x_{1}, x_{2}\right)^{\frac{1}{1+\rho}}\right]^{1+\rho}, }
\end{aligned}
$$

$$
\begin{aligned}
E_{0}^{12}\left(\rho, P_{X}\right) \triangleq & -\log \sum_{y}\left[\sum_{x_{1} \in \mathcal{X}_{1}} \sum_{x_{2} \in \mathcal{X}_{2}}\right. \\
& \left.P_{X_{1}}\left(x_{1}\right) P_{X_{2}}\left(x_{2}\right) P_{Y \mid X_{1}, X_{2}}\left(y \mid x_{1}, x_{2}\right)^{\frac{1}{1+\rho}}\right]^{1+\rho},
\end{aligned}
$$


and

$$
\begin{aligned}
\alpha_{1} & =\max _{\boldsymbol{t} \in \mathcal{T}_{q}^{n} \backslash\left\{\mathcal{T}_{q}^{n}(\mathbf{0})\right\}} \frac{\bar{S}_{1}^{n}(\boldsymbol{t})}{\left(M_{1}-1\right) B(n, \boldsymbol{t}) q^{-n}}, \\
\alpha_{2} & =\max _{\boldsymbol{t} \in \mathcal{T}_{q}^{n} \backslash\left\{\mathcal{T}_{q}^{n}(\mathbf{0})\right\}} \frac{\bar{S}_{2}^{n}(\boldsymbol{t})}{\left(M_{2}-1\right) B(n, \boldsymbol{t}) q^{-n}}, \\
\alpha_{12} & =\alpha_{1} \alpha_{2} .
\end{aligned}
$$

Here $\mathcal{T}_{q}^{n}$ is the set of possible types for $n$ elements from alphabet $\operatorname{GF}(q), \mathcal{T}_{q}^{n}(\mathbf{0})$ is the type of the all-zero codeword, $\bar{S}_{i}^{n}(\boldsymbol{t})$ is the $\operatorname{LDPC}\left(\lambda_{i}, \rho_{i} ; n\right)$ ensemble-average number of type- $t$ vectors, and $M_{i}=q^{n R_{i}}$ for $i \in\{1,2\}$.

Proof: See Appendix F

Theorem 4 presents an upper bound, which is valid for any blocklength $n$, on the ensemble-average error probability for an arbitrary DM-2-MAC when each transmitter employs a random code from the $\operatorname{LDPC}\left(\lambda_{i}, \rho_{i}, \delta_{i} ; n\right)$ ensemble. The final expression is a function of three error exponents. Note from [25] that all error exponents, $E_{p_{1}}\left(R_{1}\right), E_{p_{2}}\left(R_{2}\right)$, and $E_{p_{12}}\left(\overline{R_{1}}+R_{2}\right)$ are positive when the rate pair

$$
\left(R_{1}, R_{2}\right) \in \mathcal{R}\left(P_{X_{1}}, P_{X_{2}}\right) .
$$

Note that the quantizers $\delta_{i}(\cdot), i \in\{1,2\}$ restrict achievable input distributions $P_{X_{i}}, i \in\{1,2\}$ to be integer multiples of $\frac{1}{q}$, rate pairs $\left(R_{1}, R_{2}\right)$ that require irrational input distributions or rational input distributions with non-integer multiples of $\frac{1}{q}$ may require large alphabet size $q$ to closely approximate the desired input distributions.

However, restricting the ensemble from standard i.i.d. random codes to LDPC codes incurs the rate offset penalties $\frac{\log \alpha_{1}}{n}, \frac{\log \alpha_{2}}{n}$, and $\frac{\log \alpha_{12}}{n}$.

To eliminate these rate offsets, one can apply the expurgation technique from Lemma 5 in Appendix $C$ to remove codes with small minimum distances for both $\operatorname{LDPC}\left(\lambda_{1}, \rho_{1}, \delta_{1} ; n\right)$ and $\operatorname{LDPC}\left(\lambda_{2}, \rho_{2}, \delta_{2} ; n\right)$ ensembles. The same argument in Theorem 2 can then be used to prove these rate offsets can be made arbitrarily small, with large enough blocklength $n, \rho_{1}$, and $\rho_{2}$. More precisely, when $\rho_{1}=\kappa_{1} n$ and $\rho_{2}=\kappa_{2} n$ for some $\kappa_{1}$ and $\kappa_{2}$ that decay no more quickly than $\Theta\left(\frac{\log n}{n}\right)$, these rate offsets decay as $O\left(\frac{\log n}{n}\right)$ provided that (see Appendix $\mathrm{D}$ for details). Therefore, the proposed quantized cosetshifted LDPC MAC codes are capable of achieving any rate pair $\left(R_{1}, R_{2}\right) \in \mathcal{R}\left(P_{X_{1}}, P_{X_{2}}\right)$.

The true capacity region for the DM-2-MAC is the convex closure of the set

$$
\mathcal{R} \triangleq \bigcup_{P_{X_{1}} P_{X_{2}}} \mathcal{R}\left(P_{X_{1}}, P_{X_{2}}\right)
$$

for all $P_{X_{1}} P_{X_{2}}$. To justify any rate pair in the capacity region $\mathcal{R}$ is achievable with the proposed quantized coset-shifted LDPC MAC codes, one can apply the standard time sharing technique [26] to introduce an auxiliary random variable $W \in \mathcal{W}$ with $|\mathcal{W}| \leq 2$. The two quantizers are then defined to be dependent on the auxiliary random variable, giving the distribution $P_{W}(w) P_{X_{1} \mid W}\left(x_{1} \mid w\right) P_{X_{2} \mid W}\left(x_{2} \mid w\right)$.

\section{Finite-Blocklength Bound via Error Exponent}

We next seek to relate Gallager's error exponent bound [6] to the dispersion-style bound [5], which accurately approximates the maximal achievable rate in the non-asymptotic regime.

We begin with a short overview of both results. In [5], Polyanskiy et al. bound the maximal code size $M^{*}(n, \epsilon)$ achievable with error probability $\epsilon$ and blocklength $n$ as a function of the channel capacity $C$, the channel dispersion $V$, and the inverse complementary Gaussian $\operatorname{CDF} Q^{-1}(\cdot)$. The resulting bound is reproduced as Theorem 5 below.

Theorem 5: ([5. Cor. 51]). For a DM-PPC, if $0<\epsilon \leq \frac{1}{2}$, then

$$
\frac{\log _{2} M^{*}(n, \epsilon)}{n} \geq C-\sqrt{\frac{V_{\min }}{n}} Q^{-1}(\epsilon)+O\left(\frac{1}{n}\right),
$$

where $V_{\min }$ is the minimal channel dispersion over all capacity-achieving channel input distributions.

The same paper also bounds the dispersion $V$ for DM-PPCs.

Theorem 6: ([5, Th. 50]). Consider a DM-PPC with input alphabet $\mathcal{X}$ and output alphabet $\mathcal{Y}$ such that $\min \{|\mathcal{X}|,|\mathcal{Y}|\}>$ 2. Then

$$
V \leq 2 \log _{2}^{2}(\min \{|\mathcal{X}|,|\mathcal{Y}|\})-C^{2} .
$$

For DM-PPCs with $\min \{|\mathcal{X}|,|\mathcal{Y}|\}=2$, the upper bound becomes

$$
V \leq 1.2 \log _{2}^{2} e-C^{2} .
$$

While Theorems 5 and 6 together bound the maximal code size, and therefore rate, as a function of the blocklength $n$ and error probability $\epsilon$, Gallager's error exponent bounds error probability as a function of the blocklength $n$ and rate $R$, as described in Theorem 7

Theorem 7: ([6. Th. 5.6.2., Corollary 1]). Given a DM-PPC with transition probability $P_{Y \mid X}$, for any positive integer $n$ and positive number $R$, consider the ensemble of length- $n$ block codes, in which each symbol of each codeword $m$, $m \in\left[\left\lceil e^{n R}\right\rceil\right]$, is independently drawn according to $P_{X}$. The ensemble-average probability of decoding error using ML decoding satisfies

$$
\bar{P}_{e} \leq e^{-n E_{p}(R)},
$$

where $E_{p}(R)=\max _{0 \leq \rho \leq 1}\left[E_{0}\left(\rho, P_{X}\right)-\rho R\right]$ is Gallager's random coding error exponent for input distribution $P_{X}$ defined in Theorem 1

Remark 8: Note that the bound (42) also applies to an ensemble of random linear codes, see [6. Section 6.2]. Therefore, there is no loss in performance for using only linear codes in Gallager's approach.

Theorem 8: ([6, Exercise 5.23]). Given a DM-PPC with transition probability $P_{Y \mid X}$, 42) can be bound as

$$
\bar{P}_{e} \leq e^{\left[-n \frac{(C-R)^{2}}{8 / e^{2}+4\left(\log _{e}|\mathcal{Y}|\right)^{2}}\right]}, \forall R \in[0, C],
$$

where $|\mathcal{Y}|$ is the size of the output alphabet.

This bound results from a power series expansion of $E_{p}(R)$ evaluated at the capacity achieving input distribution $P_{X}$. 
Bounding the second derivative $E_{0}^{\prime \prime}\left(\rho, P_{X}\right)$ with respect to $\rho$ from below yields the given lower bound on $E_{p}(R)$.

Proof: An outline is shown in Appendix G

Note that a stronger bound can be proved by following the approach outlined in [6. Exercise 5.23], as shown in Corollary 1 below.

Corollary 1: Given a DM-PPC with transition probability $P_{Y \mid X}$, 42] can be bound as

$$
\bar{P}_{e} \leq e^{\left[-n \frac{(C-R)^{2}}{8 / e^{2}+2\left(\log _{e}|\mathcal{Y}|\right)^{2}-2 R_{c r}^{2}}\right]},
$$

for $R \in\left[\max \left\{0, C-\left(\frac{4}{e^{2}}+\log _{e}^{2}|\mathcal{Y}|-R_{c r}^{2}\right)\right\}, C\right]$. Here $R_{c r} \triangleq$ $E_{0}^{\prime}\left(1, P_{X}\right)$ is the critical rate [6. Eq. (5.6.30)].

Remark 9: Gallager's error exponent $E_{p}(R)$ is a lower (achievability) bound on the true error exponent (known as the reliability function [6, eq. 5.8.8]) for a given $R$. A key property of the critical rate $R_{c r}$ is that for rates $R \in\left(R_{c r}, C\right)$, Gallager's error exponent $E_{p}(R)$ equals the sphere-packing upper bound (converse) of the true error exponent $[6$. Section 5.8]).

Let the ensemble-average error probability $\bar{P}_{e}$ be the targeted error probability $\epsilon$. The stronger bound (44) can be rearranged as

$$
R \geq C-\sqrt{\frac{8 / e^{2}+2\left(\log _{e}|\mathcal{Y}|\right)^{2}-2 R_{c r}^{2}}{n} \log _{e} \frac{1}{\epsilon}} .
$$

Polyanskiy's and Gallager's strategies yield random coding achievability bounds. For Polyanskiy's approach, Theorem 5 bounds the rate as a function of the channel's capacity and dispersion, while Theorem 6 bounds the dispersion of a DMPPC in terms of the input and output alphabet sizes of the channel. In Gallager's approach, Theorem 8 bounds the error probability using the capacity and (only) the output alphabet size. Comparing these two approaches yield the following observations.

- The first order term in 39) (Polyanskiy's approach) and (45) (Gallager's approach) are both the channel capacity $C$.

- Polyanskiy's approach yields a tighter coefficient in the second-order term. Precisely, the second-order terms in (39) and (45) are both $O(\sqrt{1 / n})$. However, the upper bound on the coefficient in Polyanskiy's approach $\sqrt{39}$ is

$$
2 \log _{2}^{2}(\min \{|\mathcal{X}|,|\mathcal{Y}|\})-C^{2}(C \text { in bits }),
$$

which is tighter than the coefficient in Gallager's approach (45)

$$
\begin{aligned}
& 8 / e^{2}+2\left(\log _{e}|\mathcal{Y}|\right)^{2}-2 R_{c r}^{2} \quad\left(R_{c r} \text { in nats }\right) \\
= & \frac{8 \log _{2}^{2}(e)}{e^{2}}+2\left(\log _{2}|\mathcal{Y}|\right)^{2}-2 R_{c r}^{2}\left(R_{c r} \text { in bits }\right) .
\end{aligned}
$$

Therefore, we conclude that the error-exponent approach yields a sub-optimal coefficient in the $\sqrt{1 / n}$ term.

- Gallager's approach yields a better scaling at small error probability $\epsilon$. More precisely, for a given targeted error probability $\epsilon$, the $\sqrt{1 / n}$ term in (39) (Polyanskiy's approach) scales as $Q^{-1}(\epsilon)$, while the corresponding term in 45) (Gallager's approach) scales as $\sqrt{\log _{e} \frac{1}{\epsilon}}$.

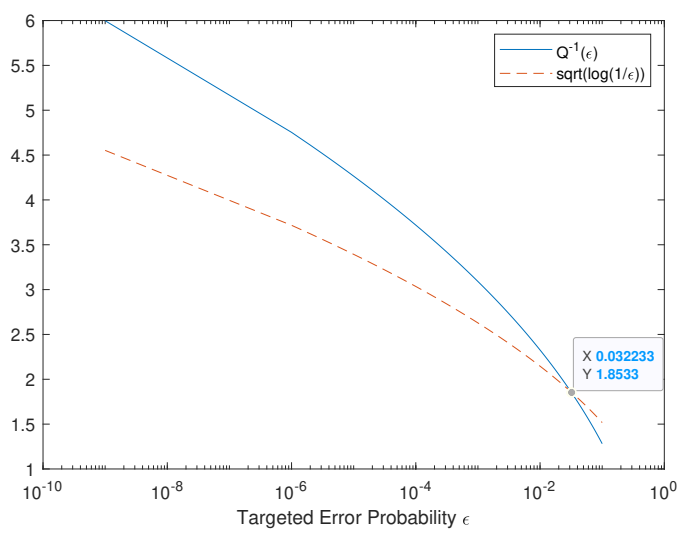

Fig. 2. Comparison between $Q^{-1}(\epsilon)$ with $\sqrt{\log _{e} \frac{1}{\epsilon}}$.

A comparison between these scaling terms is shown in Figure 2, which confirms the advantage of the errorexponent approach (originally designed for analyzing exponentially small error) at small $\epsilon$.

Applying the outcome of the error-exponent approach to Theorem 2, we obtain the following achievability result.

Theorem 9: Let $P_{Y \mid X}$ be the transition probability for a symmetrical DM- $K$-MAC with input alphabet $\mathcal{X}=\mathcal{U}^{K}$ and output alphabet $\mathcal{Y}$. Let $C, P_{U}$, and $\delta(\cdot)$ be defined as in Theorem 11. Then there exist LDPC parameters $(\lambda, \rho)$ for which the expurgated ensemble $\mathrm{LDPC}_{K}-\mathrm{Ex}_{\sigma}(\lambda, \rho, \delta ; n)$ contains at least one code with average error probability less than $\epsilon$ under ML decoding and

$$
\begin{aligned}
& R \geq \\
& \frac{1}{K}\left[K C-\sqrt{\frac{8 \log ^{2}(e) / e^{2}+2(\log |\mathcal{Y}|)^{2}}{n} \log \frac{1}{\epsilon}}-\frac{\log \alpha_{\mathrm{ex}}}{n}\right],
\end{aligned}
$$

where

$$
\alpha_{\mathrm{ex}}=\max _{\boldsymbol{\theta} \in J_{\sigma}} \frac{\bar{S}_{\mathrm{ex}, \sigma}^{n}(n \boldsymbol{\theta})}{\left(M^{K}-1\right) B(n, n \boldsymbol{\theta}) q^{-n K}},
$$

and $\bar{S}_{\mathrm{ex}, \sigma}^{n}(n \boldsymbol{\theta})$ is the average spectrum of the expurgated ensemble. If $\rho=\kappa n$ and $\kappa$ approaches zero no more quickly than $\Theta\left(\frac{\log n}{n}\right)$, then $\frac{\log \alpha_{\mathrm{ex}}}{n}=O\left(\frac{\log n}{n}\right)$.

Remark 10: The error-exponent approach imposes a suboptimal $\sqrt{1 / n}$ second-order term even for codes drawn i.i.d. from $P_{X}$. The additional penalty for using LDPC codes instead of i.i.d. $P_{X}$ codes is $\frac{\log \alpha_{\mathrm{ex}}}{n}$, which is $O\left(\frac{\log n}{n}\right)$ for large enough $\rho$ as shown in the proof of Theorem 2 . This observation raises the question of whether the dispersion-style approach can be applied to LDPC code, and, if so, whether the LDPC code can achieve the optimal second-order term. To answer this question, we first review the derivation of dispersion-style bound and tighten a prior result for the PPCs. 


\section{RCU BOUNDS FOR I.I.D. CODES}

\section{A. RCU Bound for I.I.D. Code on the DM-PPC}

In [5], Polyanskiy, Poor, and Verdú study the PPC using techniques including the RCU bound, the dependency-testing (DT) bound, and the $\kappa \beta$ bound. We here build on the RCU bound, which employs the optimal ML decoder.

Theorem 10, below, presents a slightly more general version of the non-asymptotic RCU bound from [5, Th. 16]. The key difference between Theorem 10 and [5, Th. 16] is that the RCU bound in [5] requires all codewords to be drawn i.i.d. according to $P_{X}$ while Theorem 10 requires only that the marginal distribution on each codeword equals $P_{X}$. For example, Theorem 10 can be applied to codes whose codewords are dependent, in which case the joint distribution $P_{X \bar{X}}(a, b)$ on a pair of codewords $X$ and $\bar{X}$ is not equal to $P_{X}(a) P_{X}(b)$ for some $(a, b) \in \mathcal{X}^{2}$.

Theorem 10: (RCU bound, modified from [5, Th. 16]) Consider an ensemble of codes with $M$ codewords drawn according to some $P_{X(1) X(2) \ldots X(M)}$ such that

$$
P_{X(\mathcal{A})}=P_{X(\mathcal{B})}, \forall \mathcal{A}, \mathcal{B} \subseteq[M] \text { s.t. }|\mathcal{A}|=|\mathcal{B}| .
$$

Under ML decoding, the ensemble-average error probability $\epsilon$ satisfies

$$
\epsilon \leq \mathbb{E}[\min \{1,(M-1) \operatorname{Pr}[i(\bar{X} ; Y) \geq i(X ; Y) \mid X, Y]\}],
$$

where

$$
\begin{aligned}
P_{X \bar{X} Y}(a, b, c) & =P_{X \bar{X}}(a, b) P_{Y \mid X}(c \mid a) \\
P_{X \bar{X}}(a, b) & =P_{X(1) X(2)}(a, b) .
\end{aligned}
$$

Proof: Denote the conditional error probability given that the $j$-th codeword is sent by $\epsilon_{j}$, then the average error probability is

$$
\epsilon_{\mathrm{avg}}=\frac{1}{M} \sum_{j=1}^{M} \epsilon_{j} .
$$

By the symmetry of both the code design (implied by (48)) and the ML decoder

$$
\mathbb{E}\left[\epsilon_{\text {avg }}\right]=\mathbb{E}\left[\epsilon_{1}\right],
$$

where the expectation is taken over the random codebook design.

The ML decoder $g(\cdot)$ gives

$$
\begin{aligned}
g(y) & =\underset{j \in[M]}{\arg \max } P_{Y \mid X}(y \mid X(j)) \\
& =\underset{j \in[M]}{\arg \max } \frac{P_{Y \mid X}(y \mid X(j))}{P_{Y}(y)} \\
& =\underset{j \in[M]}{\arg \max } i(X(j) ; y) .
\end{aligned}
$$

For the case of a tie, the decoder chooses uniformly at random among the most probable codewords.

Given that the first codeword $X(1)$ is transmitted, an error or tie occurs when the channel output is some value $y$ for which

$$
\exists j \in[M] \backslash\{1\} \text {, s.t. } i(X(j) ; y) \geq i(X(1) ; y) .
$$

Therefore, $\mathbb{E}\left[\epsilon_{1}\right]$ can be bounded from above as

$$
\begin{aligned}
& \mathbb{E}\left[\epsilon_{1}\right] \\
& \leq \operatorname{Pr}\left[\bigcup_{j=2}^{M}\{i(X(j) ; Y) \geq i(X(1) ; Y)\}\right] \\
& =\mathbb{E}\left[\operatorname{Pr}\left[\bigcup_{j=2}^{M}\{i(X(j) ; Y) \geq i(X(1) ; Y)\} \mid X(1), Y\right]\right] \\
& \leq \mathbb{E}[\min \{1,(M-1) \\
& \quad \operatorname{Pr}[\{i(X(2) ; Y) \geq i(X(1) ; Y)\} \mid X(1), Y]\}],
\end{aligned}
$$

where (58) is an inequality as the decoder might resolve some ties correctly, (59) follows from the law of iterated expectation, and (60) holds by union bound and the bounded nature of probability. Note that 60 follows since all terms in the union bound are equal as the conditional distribution of all $X(2), \ldots, X(M)$ given the transmitted $X(1)$ are the same by the symmetry of code design.

It is useful to notice that the bound in Theorem 10 equation (49) takes the same form for all choices of $P_{X(1) X(2) \ldots X(M)}$ satisfying (48), but that the evaluation of that bound varies with the precise dependence or independence of $X_{1}$ and $X_{2}$ or, equivalently, $X$ and $\bar{X}$ under the chosen code distribution. For example, the value of $\operatorname{Pr}[i(\bar{X} ; Y) \geq i(X ; Y) \mid X, Y]$ is exactly one when $\bar{X}=X$ with probability one, but it is less than or equal to one for other choices of $P_{X(1) X(2)}$. While we begin by evaluating Theorem 10 under the case of independent codewords $\left(P_{X(1) X(2) \ldots X(M)}=\left(P_{X}\right)^{M}\right)$, we require the more general form for evaluating LDPC codes, where codewords are not drawn i.i.d. but instead result from a shared Tanner graph.

We now follow the approach in [27, Th. 5] to apply Theorem 10 and two other important theorems to prove the achievability bound in Theorem 11. The given analysis tightens the achievability result from a third-order term $O(\log n)$ in [5. Th. 49] to $\frac{1}{2} \log n-O(1)$, yielding a result that matches the corresponding converse bound [5. Th. 48] up to the third order.

Theorem 11: (Random coding finite-blocklength bound and asymptotic third-order-optimal achievability for the PPC). Consider a DM-PPC with channel transition probability $P_{Y \mid X}$ and capacity achieving distribution $P_{X}$. If each symbol of each codeword is drawn i.i.d. according to $P_{X}$, then there exists a blocklength- $n$ code with $M$ codewords and average error probability $\epsilon$ such that for any blocklength $n$

$$
\epsilon \leq \mathbb{E}\left[\min \left\{1, M \frac{A\left(P_{X}\right)}{\sqrt{n}} \exp \left(-i\left(X^{n} ; Y^{n}\right)\right)\right\}\right],
$$

and for large enough $n$

$$
\frac{\log M}{n} \geq C-\sqrt{\frac{V\left(P_{X}\right)}{n}} Q^{-1}(\epsilon)+\frac{\log n}{2 n}-O\left(\frac{1}{n}\right),
$$

provided the following moment assumptions are satisfied when $X \sim P_{X}$

$$
\begin{aligned}
I\left(P_{X}\right) & >0, \\
V\left(P_{X}\right) & >0,
\end{aligned}
$$




$$
\begin{aligned}
V^{Y}\left(P_{X}\right) & >0, \\
T\left(P_{X}\right) & <\infty,
\end{aligned}
$$

where

$$
\begin{aligned}
I\left(P_{X}\right) & =\mathbb{E}[i(X ; Y)], \\
V\left(P_{X}\right) & =\operatorname{Var}[i(X ; Y)], \\
V^{Y}\left(P_{X}\right) & =\operatorname{Var}[i(X ; Y) \mid Y], \\
T\left(P_{X}\right) & =\mathbb{E}\left[|i(X ; Y)-\mathbb{E}[i(X ; Y)]|^{3}\right], \\
B\left(P_{X}\right) & \triangleq \frac{C_{0} T\left(P_{X}\right)}{V\left(P_{X}\right)^{3 / 2}} \\
A\left(P_{X}\right) & \triangleq 2\left(\frac{\log 2}{\sqrt{2 \pi V\left(P_{X}\right)}}+2 B\left(P_{X}\right)\right) .
\end{aligned}
$$

The proof of Theorem 11 relies on the Berry-Esséen inequality and [5, Lemma 47], as stated in Theorem 12 and Lemma 1, respectively.

Theorem 12: (Berry-Esséen Theorem, [28, Chapter XVI.5]). Let $Z_{1}, \ldots, Z_{n}$ be a sequence of independent random variables with distribution $Z_{j} \sim P_{Z_{j}}$. Assume that

$$
\begin{aligned}
\mathbb{E}\left[Z_{j}\right] & =\mu_{j}, \forall j \in\{1, \ldots, n\}, \\
\mu & =\frac{1}{n} \sum_{j=1}^{n} \mu_{j}, \\
V & =\frac{1}{n} \sum_{j=1}^{n} \operatorname{Var}\left[Z_{j}\right]>0, \\
T & =\frac{1}{n} \sum_{j=1}^{n} \mathbb{E}\left[\left|Z_{j}-\mu_{j}\right|^{3}\right]<\infty .
\end{aligned}
$$

Then for any $-\infty<\lambda<\infty$ and $n \geq 1$

$$
\left|\operatorname{Pr}\left[\sum_{j=1}^{n} Z_{j} \geq n\left(\mu+\lambda \sqrt{\frac{V}{n}}\right)\right]-Q(\lambda)\right| \leq \frac{C_{0} T}{V^{3 / 2}} \frac{1}{\sqrt{n}},
$$

where $C_{0} \leq 0.5583$ for independent random variables, and $C_{0} \leq 0.4690$ for i.i.d. random variables [29].

The exact value of $C_{0}$ does not affect the results in this paper. We employ $C_{0}=0.5583$ even for the i.i.d. case.

Lemma 1: ([5, Lemma 47]). Let $Z_{1}, \ldots, Z_{n}$ be a sequence of independent random variables with distribution $Z_{j} \sim P_{Z_{j}}$. Assume

$$
\begin{aligned}
V & =\frac{1}{n} \sum_{j=1}^{n} \operatorname{Var}\left[Z_{j}\right]>0, \\
T & =\frac{1}{n} \sum_{j=1}^{n} \mathbb{E}\left[\left|Z_{j}-\mu_{j}\right|^{3}\right]<\infty .
\end{aligned}
$$

Then for any constant $\zeta$

$$
\begin{aligned}
& \mathbb{E}\left[\exp \left\{-\sum_{j=1}^{n} Z_{j}\right\} \mathbb{1}\left\{\sum_{j=1}^{n} Z_{j} \geq \zeta\right\}\right] \\
& \leq 2\left(\frac{\log 2}{\sqrt{2 \pi V}}+2 \frac{C_{0} T}{V^{3 / 2}}\right) \frac{1}{\sqrt{n}} \exp (-\zeta) .
\end{aligned}
$$

The proof of Theorem 11 follows the proof of a similar source coding argument in [27, Th. 5].

Proof of Theorem 11. Setting $X=X^{n}, \bar{X}=\bar{X}^{n}, Y=$ $Y^{n}$ in Theorem 10, we note that the ensemble-average error probability $\epsilon^{\prime}$ satisfies

$$
\epsilon^{\prime} \leq \mathbb{E}\left[\min \left\{1, M \operatorname{Pr}\left[i\left(\bar{X}^{n} ; Y^{n}\right) \geq i\left(X^{n} ; Y^{n}\right) \mid X^{n}, Y^{n}\right]\right\}\right],
$$

where

$$
\begin{aligned}
P_{X^{n} \bar{X}^{n} Y^{n}}\left(x^{n}, \bar{x}^{n}, y^{n}\right) & =P_{X^{n}, \bar{X}^{n}}\left(x^{n}, \bar{x}^{n}\right) P_{Y^{n} \mid X^{n}}\left(y^{n} \mid x^{n}\right) \\
& =P_{X^{n}}\left(x^{n}\right) P_{X^{n}}\left(\bar{x}^{n}\right) P_{Y^{n} \mid X^{n}}\left(y^{n} \mid x^{n}\right),
\end{aligned}
$$

as the codewords are drawn i.i.d. according to $P_{X^{n}}=P_{X}^{n}$.

Denote for brevity

$$
\begin{aligned}
& I_{n} \triangleq i\left(X^{n} ; Y^{n}\right)=\sum_{j=1}^{n} i\left(X_{j} ; Y_{j}\right) \\
& \bar{I}_{n} \triangleq i\left(\bar{X}^{n} ; Y^{n}\right)=\sum_{j=1}^{n} i\left(\bar{X}_{j} ; Y_{j}\right),
\end{aligned}
$$

where $V\left(P_{X}\right)$ and $T\left(P_{X}\right)$ are the second-order moment and third-order central moment of the information density, respectively as defined in (68) and $(70)$, and $B\left(P_{X}\right)$ and $A\left(P_{X}\right)$ are positive and finite by the moment assumptions (64)- 666.

Since the codewords are drawn i.i.d. according to $P_{X^{n}}=P_{X}^{n}, \bar{X}^{n}$ is independent of $\left(X^{n}, Y^{n}\right)$, and if $P_{Y^{n} \mid X^{n}}\left(Y^{n} \mid \bar{x}^{n}\right)>0$, then

$$
\begin{aligned}
& \operatorname{Pr}\left[\bar{X}^{n}=\bar{x}^{n} \mid X^{n}, Y^{n}\right] \\
& =\operatorname{Pr}\left[\bar{X}^{n}=\bar{x}^{n}\right] \\
& =P_{X^{n}}\left(\bar{x}^{n}\right) \frac{P_{Y^{n} \mid X^{n}}\left(Y^{n} \mid \bar{x}^{n}\right)}{P_{Y^{n}}\left(Y^{n}\right)} \frac{P_{Y^{n}}\left(Y^{n}\right)}{P_{Y^{n} \mid X^{n}}\left(Y^{n} \mid \bar{x}^{n}\right)} \\
& =\operatorname{Pr}\left[X^{n}=\bar{x}^{n} \mid Y^{n}\right] \exp \left\{-i\left(\bar{x}^{n} ; Y^{n}\right)\right\} .
\end{aligned}
$$

If $P_{Y^{n} \mid X^{n}}\left(Y^{n} \mid \bar{x}^{n}\right)=0$, then $\operatorname{Pr}\left[\bar{X}^{n}=\bar{x}^{n} \mid X^{n}, Y^{n}\right]=$ $\operatorname{Pr}\left[\bar{X}^{n}=\bar{x}^{n}\right]$. However, since $P_{Y^{n} \mid X^{n}}\left(Y^{n} \mid \bar{x}^{n}\right)=0$ implies $\bar{I}_{n}=-\infty$, we only sum over $\bar{x}^{n}$ such that $P_{Y^{n} \mid X^{n}}\left(Y^{n} \mid \bar{x}^{n}\right)>$ 0 in the following derivation.

Fix some constant $\zeta$. Using (88) and summing over all $\bar{x}^{n}$ such that $\bar{I}_{n} \geq \zeta$ gives

$$
\operatorname{Pr}\left[\bar{I}_{n} \geq \zeta \mid Y^{n}\right]=\mathbb{E}\left[\exp \left\{-I_{n}\right\} \mathbb{1}\left\{I_{n} \geq \zeta\right\} \mid Y^{n}\right] .
$$

Given $Y^{n}, I_{n}$ is a sum of independent random variables. Note from 65 that $V^{Y}\left(P_{X}\right)>0$. Taking $Z_{j}=-i\left(X_{j} ; Y_{j}\right)$, Lemma 1 implies

$$
\operatorname{Pr}\left[\bar{I}^{n} \geq \zeta \mid Y^{n}\right] \leq \frac{A\left(P_{X}\right)}{\sqrt{n}} \exp (-\zeta) .
$$

Therefore,

$$
\begin{aligned}
\epsilon^{\prime} \leq & \mathbb{E}\left[\min \left\{1, M \frac{A\left(P_{X}\right)}{\sqrt{n}} \exp \left(-I_{n}\right)\right\}\right] \\
= & 1 \cdot \operatorname{Pr}\left[I_{n}<\log \frac{M A\left(P_{X}\right)}{\sqrt{n}}\right] \\
& +\mathbb{E}\left[\frac{M A\left(P_{X}\right)}{\sqrt{n}} \exp \left(-I_{n}\right) \mathbb{1}\left\{I_{n} \geq \log \frac{M A\left(P_{X}\right)}{\sqrt{n}}\right\}\right]
\end{aligned}
$$




$$
\begin{aligned}
= & \operatorname{Pr}\left[I_{n}<\log \frac{M A\left(P_{X}\right)}{\sqrt{n}}\right] \\
& +\frac{M A\left(P_{X}\right)}{\sqrt{n}} \mathbb{E}\left[\exp \left(-I_{n}\right) \mathbb{1}\left\{I_{n} \geq \log \frac{M A\left(P_{X}\right)}{\sqrt{n}}\right\}\right] \\
\leq & \operatorname{Pr}\left[I_{n}<\log M+\log A\left(P_{X}\right)-\frac{1}{2} \log n\right] \\
& +\frac{M A\left(P_{X}\right)}{\sqrt{n}} \cdot \frac{A\left(P_{X}\right)}{\sqrt{n}} \cdot \exp \left(-\log \frac{M A\left(P_{X}\right)}{\sqrt{n}}\right) \\
= & \operatorname{Pr}\left[I_{n}<\log M+\log A\left(P_{X}\right)-\frac{1}{2} \log n\right]+\frac{A\left(P_{X}\right)}{\sqrt{n}}
\end{aligned}
$$

where (92) separates the two possible outcomes of the minimization in (91), and $94 \mathrm{p}$ applies Lemma 1 to the last term in 93. with $\zeta=\log \frac{M A\left(P_{X}\right)}{\sqrt{n}}$.

Recall from 677) that $I\left(P_{X}\right)=\mathbb{E}[i(X ; Y)]$ and that, under our i.i.d. codeword design, $\mathbb{E}\left[I^{n}\right]=n I\left(P_{X}\right)$. Therefore, setting

$$
\begin{aligned}
\log M= & n I\left(P_{X}\right)+\frac{1}{2} \log n-\log A\left(P_{X}\right) \\
& +\sqrt{n V\left(P_{X}\right)} Q^{-1}\left(1-\left(\epsilon-\frac{B\left(P_{X}\right)+A\left(P_{X}\right)}{\sqrt{n}}\right)\right),
\end{aligned}
$$

we have

$$
\begin{aligned}
\operatorname{Pr} & {\left[I_{n}<\log M+\log A\left(P_{X}\right)-\frac{1}{2} \log n\right] } \\
= & 1-\operatorname{Pr}\left[I_{n} \geq n I\left(P_{X}\right)\right. \\
& \left.+\sqrt{n V\left(P_{X}\right)} Q^{-1}\left(1-\left(\epsilon-\frac{B\left(P_{X}\right)+A\left(P_{X}\right)}{\sqrt{n}}\right)\right)\right] \\
\leq & 1-\left(-\frac{B\left(P_{X}\right)}{\sqrt{n}}\right. \\
& \left.+Q\left(Q^{-1}\left(1-\left(\epsilon-\frac{B\left(P_{X}\right)+A\left(P_{X}\right)}{\sqrt{n}}\right)\right)\right)\right) \\
= & 1-\left(1-\epsilon+\frac{A\left(P_{X}\right)}{\sqrt{n}}\right) \\
= & \epsilon-\frac{A\left(P_{X}\right)}{\sqrt{n}},
\end{aligned}
$$

where 98 follows from $\operatorname{Pr}[X<a]=1-\operatorname{Pr}[X \geq a]$, and 990 holds by applying the Berry-Esséen Theorem (Theorem 12 to the last term in 98 with $\sum_{j=1}^{n} Z_{j}=I_{n}$ and $\lambda=Q^{-1}\left(1-\left(\epsilon-\frac{B\left(P_{X}\right)+A\left(P_{X}\right)}{\sqrt{n}}\right)\right)$. Note that the BerryEsséen Theorem is given in the form $|a-b| \leq c$, and $99 \mid$ applies the lower bound, i.e., $a-b \geq-c$.

Plugging (101) into 95) gives

$$
\epsilon^{\prime} \leq \epsilon-\frac{A\left(P_{X}\right)}{\sqrt{n}}+\frac{A\left(P_{X}\right)}{\sqrt{n}}=\epsilon,
$$

which gives an achievability bound $\log M \geq n I\left(P_{X}\right)+\frac{1}{2} \log n-\log A\left(P_{X}\right)$

$$
\begin{aligned}
& +\sqrt{n V\left(P_{X}\right)} Q^{-1}\left(1-\left(\epsilon-\frac{B\left(P_{X}\right)+A\left(P_{X}\right)}{\sqrt{n}}\right)\right) \\
= & n I\left(P_{X}\right)+\frac{1}{2} \log n-\log A\left(P_{X}\right) \\
& -\sqrt{n V\left(P_{X}\right)} Q^{-1}\left(\epsilon-\frac{B\left(P_{X}\right)+A\left(P_{X}\right)}{\sqrt{n}}\right),
\end{aligned}
$$

where (104) follows from the property of inverse $Q$ function, $Q^{-1}(1-\epsilon)=-Q^{-1}(\epsilon)$ for all $0<\epsilon<1$.

Finally, we use the 1st-order Taylor bound and the inverse function theorem as in [27, Eq. (65)-(69)] to derive the bounds

$$
\begin{aligned}
& Q^{-1}\left(\epsilon-\frac{B\left(P_{X}\right)+A\left(P_{X}\right)}{\sqrt{n}}\right) \\
& \leq Q^{-1}(\epsilon)+\frac{B\left(P_{X}\right)+A\left(P_{X}\right)}{\sqrt{n} \phi\left(\Phi^{-1}\left(\Phi\left(Q^{-1}(\epsilon)\right)+\frac{B\left(P_{X}\right)+A\left(P_{X}\right)}{\sqrt{n}}\right)\right)},
\end{aligned}
$$

when $\epsilon \leq \frac{1}{2}$ and $n>\left(\frac{B\left(P_{X}\right)+A\left(P_{X}\right)}{\epsilon}\right)^{2}$, and

$$
\begin{array}{r}
Q^{-1}\left(\epsilon-\frac{B\left(P_{X}\right)+A\left(P_{X}\right)}{\sqrt{n}}\right) \\
\leq Q^{-1}(\epsilon)+\frac{B\left(P_{X}\right)+A\left(P_{X}\right)}{\sqrt{n} \phi\left(Q^{-1}(\epsilon)\right)},
\end{array}
$$

when $\epsilon>\frac{1}{2}$ and $n>\left(\frac{B\left(P_{X}\right)+A\left(P_{X}\right)}{\epsilon-1 / 2}\right)^{2}$. Recall here that $\Phi(\cdot)$ and $\phi(\cdot)$ are the CDF and PDF for the standard Gaussian distribution.

By choosing $P_{X}$ to be the capacity achieving distribution, we obtain the existence of an $M(n, \epsilon)$ code with

$$
\frac{\log M}{n} \geq C-\sqrt{\frac{V\left(P_{X}\right)}{n}} Q^{-1}(\epsilon)+\frac{\log n}{2 n}-O\left(\frac{1}{n}\right) .
$$

\section{B. RCU Bound for i.i.d. Code on the DM-2-MAC}

In this section, we first extend the RCU bound from the PPC to the MAC with two transmitters. We then present an asymptotic achievability result based on the two-user RCU bound. Our argument follows the multiple access source coding proof in [27, Th. 11] and is similar to [24, Th. 1]. The results generalize to MACs with more than two transmitters. We then present an asymptotic achievability result based on the two-user RCU bound. The bound improves the thirdorder MAC achievability bound $-O\left(\frac{\log n}{n}\right) \mathbf{1}$ in [30], and the best prior MAC achievability bound $-\nu \frac{\log n}{n} 1$ in [20], with $\nu \geq 2\left|\mathcal{X}_{1}\right|\left|\mathcal{X}_{2}\right||\mathcal{Y}|$, to $+\frac{\log n}{2 n} \mathbf{1}-O\left(\frac{1}{n}\right) \mathbf{1}$.

Consider a two-user MAC, $\left(\mathcal{X}_{1} \times \mathcal{X}_{2}, P_{Y \mid X_{1}, X_{2}}, \mathcal{Y}\right)$. An $\left(M_{1}, M_{2}, \epsilon\right)$ code is defined by two encoding functions

$$
\begin{array}{ll}
f_{1}: & {\left[M_{1}\right] \rightarrow \mathcal{X}_{1}} \\
f_{2}: & {\left[M_{2}\right] \rightarrow \mathcal{X}_{2}}
\end{array}
$$

and one decoding function

$$
g: \mathcal{Y}^{n} \rightarrow\left[M_{1}\right] \times\left[M_{2}\right]
$$


such that the average error probability is bounded by $\epsilon$

$$
\begin{aligned}
& \frac{1}{M_{1} M_{2}} \sum_{\substack{\left(w_{1}, w_{2}\right) \\
\in\left[M_{1}\right] \times\left[M_{2}\right]}} \operatorname{Pr}\left[g(Y) \neq\left(w_{1}, w_{2}\right) \mid\right. \\
& \left.X_{1}=f_{1}\left(w_{1}\right), X_{2}=f_{2}\left(w_{2}\right)\right] \leq \epsilon .
\end{aligned}
$$

Similarly, given a two-user MAC, $\left(\mathcal{X}_{1} \times \mathcal{X}_{2}, P_{Y \mid X_{1}, X_{2}}, \mathcal{Y}\right)$, a blocklength- $n\left(M_{1}, M_{2}, \epsilon\right)$ code for the two-user MAC, denoted as $\left(n, M_{1}, M_{2}, \epsilon\right)$, is defined by two encoding functions

$$
\begin{aligned}
& f_{1}:\left[M_{1}\right] \rightarrow \mathcal{X}_{1}^{n} \\
& f_{2}:\left[M_{2}\right] \rightarrow \mathcal{X}_{2}^{n}
\end{aligned}
$$

and one decoding function

$$
g: \mathcal{Y}^{n} \rightarrow\left[M_{1}\right] \times\left[M_{2}\right]
$$

such that the average error probability is bounded by $\epsilon$

$$
\begin{aligned}
& \frac{1}{M_{1} M_{2}} \sum_{\substack{\left(w_{1}, w_{2}\right) \\
\in\left[M_{1}\right] \times\left[M_{2}\right]}} \operatorname{Pr}\left[g\left(Y^{n}\right) \neq\left(w_{1}, w_{2}\right) \mid\right. \\
& \left.X_{1}^{n}=f_{1}\left(w_{1}\right), X_{2}^{n}=f_{2}\left(w_{2}\right)\right] \leq \epsilon,
\end{aligned}
$$

The corresponding (finite-blocklength) rate pair for an $\left(n, M_{1}, M_{2}, \epsilon\right)$ is defined as

$$
\begin{aligned}
R_{1} & =\frac{1}{n} \log M_{1}, \\
R_{2} & =\frac{1}{n} \log M_{2} .
\end{aligned}
$$

A rate pair $\left(R_{1}, R_{2}\right)$ is said to be $(n, \epsilon)$-achievable if there exists an $\left(n, M_{1}, M_{2}, \epsilon\right)$ code. The closure of the set of all $(n, \epsilon)$-achievable rate pairs is called the $(n, \epsilon)$-achievable rate region, denoted as $\mathcal{R}_{n, \epsilon}$.

Remark 11: The definition of an $\left(n, M_{1}, M_{2}, \epsilon\right)$ code and the corresponding rate region $\mathcal{R}_{n, \epsilon}$ apply to general two-user MACs and are not restricted to the discrete or memoryless case. In this paper, we focus on the subclass of DM-2-MACs; in this case, $P_{Y^{n} \mid X_{1}^{n}, X_{2}^{n}}=P_{Y \mid X_{1}, X_{2}}^{n}$ and $\mathcal{X}_{1}, \mathcal{X}_{2}$, and $\mathcal{Y}$ are all discrete.

Theorem 13: (Two-user RCU bound, extended from [5. Th. 16]) Consider an ensemble of MAC codes with $M_{1} \times M_{2}$ codeword pairs drawn according to some $P_{X_{1}(1) \ldots X_{1}\left(M_{1}\right)} P_{X_{2}(1) \ldots X_{2}\left(M_{2}\right)}$ such that

$$
\begin{aligned}
& P_{X_{1}(\mathcal{A})}=P_{X_{1}(\mathcal{B})}, \forall \mathcal{A}, \mathcal{B} \subseteq\left[M_{1}\right] \text { s.t. }|\mathcal{A}|=|\mathcal{B}|, \\
& P_{X_{2}(\mathcal{A})}=P_{X_{2}(\mathcal{B})}, \forall \mathcal{A}, \mathcal{B} \subseteq\left[M_{2}\right] \text { s.t. }|\mathcal{A}|=|\mathcal{B}|,
\end{aligned}
$$

Under ML decoding, the ensemble-average error probability $\epsilon$ satisfies

$$
\epsilon \leq \mathbb{E}\left[\min \left\{1, V_{1}+V_{2}+V_{12}\right\}\right],
$$

where

$$
\begin{aligned}
V_{1} & =\left(M_{1}-1\right) \operatorname{Pr}\left[i\left(\bar{X}_{1} ; Y \mid X_{2}\right) \geq i\left(X_{1} ; Y \mid X_{2}\right) \mid X_{1}, X_{2}, Y\right], \\
V_{2} & =\left(M_{2}-1\right) \operatorname{Pr}\left[i\left(\bar{X}_{2} ; Y \mid X_{1}\right) \geq i\left(X_{2} ; Y \mid X_{1}\right) \mid X_{1}, X_{2}, Y\right], \\
V_{12} & =\left(M_{1}-1\right)\left(M_{2}-1\right)
\end{aligned}
$$

$$
\left.\operatorname{Pr}\left[i\left(\bar{X}_{1}, \bar{X}_{2} ; Y\right) \geq i\left(X_{1}, X_{2} ; Y\right) \mid X_{1}, X_{2}, Y\right]\right\},
$$

and

$$
\begin{aligned}
& P_{X_{1} X_{2} \bar{X}_{1} \bar{X}_{2} Y}(a, b, c, d, e)= \\
& P_{X_{1} \bar{X}_{1}}(a, c) P_{X_{2} \bar{X}_{2}}(b, d) P_{Y \mid X_{1}, X_{2}}(e \mid a, b), \\
& P_{X_{1} \bar{X}_{1}}(a, c)=P_{X_{1}(1) X_{1}(2)}(a, c) \\
& P_{X_{2} \bar{X}_{2}}(b, d)=P_{X_{2}(1) X_{2}(2)}(b, d) .
\end{aligned}
$$

Proof: Denote the random MAC codebook as

$$
\left(X_{1}(1), \ldots, X_{1}\left(M_{1}\right)\right) \times\left(X_{2}(1), \ldots, X_{2}\left(M_{2}\right)\right),
$$

where codewords $\left(X_{k}(1), \ldots, X_{k}\left(M_{k}\right)\right)$ are chosen according to $P_{X_{k}(1), \ldots, X_{k}\left(M_{k}\right)}$ for $k \in\{1,2\}$.

Denote the conditional error probability given the codeword pair $\left(X_{1}(i), X_{2}(j)\right)$ is sent as $\epsilon_{i, j}$.

The average error probability is

$$
\epsilon_{\text {avg }}=\frac{1}{M_{1} M_{2}} \sum_{(i, j) \in\left[M_{1}\right] \times\left[M_{2}\right]} \epsilon_{i, j} .
$$

By the symmetry of code design

$$
\mathbb{E}\left[\epsilon_{\text {avg }}\right]=\mathbb{E}\left[\epsilon_{1,1}\right],
$$

where the expectation is taken over the random codebook design.

The ML decoder $g(\cdot)$ gives

$$
\begin{aligned}
g(y) & =\underset{(i, j) \in\left[M_{1}\right] \times\left[M_{2}\right]}{\arg \max } P_{Y \mid X_{1}, X_{2}}\left(y \mid X_{1}(i), X_{2}(j)\right) \\
& =\underset{(i, j) \in\left[M_{1}\right] \times\left[M_{2}\right]}{\arg \max } \frac{P_{Y \mid X_{1}, X_{2}}\left(y \mid X_{1}(i), X_{2}(j)\right)}{P_{Y}(y)} \\
& =\underset{(i, j) \in\left[M_{1}\right] \times\left[M_{2}\right]}{\arg \max } i\left(X_{1}(i), X_{2}(j) ; y\right),
\end{aligned}
$$

and ties are broken uniformly at random.

Given that codeword pair $\left(X_{1}(1), X_{2}(1)\right)$ is transmitted, an error or tie occurs if

$$
\begin{array}{r}
\exists(i, j) \in\left[M_{1}\right] \times\left[M_{2}\right] \backslash\{1,1\}, \\
\text { s.t. } i\left(X_{1}(i), X_{2}(j) ; y\right) \geq i\left(X_{1}(1), X_{2}(1) ; y\right) .
\end{array}
$$

Note that condition (124) can be equivalently written as the union of the following events

1) $\exists i \in\left[M_{1}\right] \backslash\{1\}$, s.t.

2) $\exists j \in\left[M_{2}\right] \backslash\{1\}$, s.t.

$$
i\left(X_{1}(i), X_{2}(1) ; y\right) \geq i\left(X_{1}(1), X_{2}(1) ; y\right) ;
$$

$$
i\left(X_{1}(1), X_{2}(j) ; y\right) \geq i\left(X_{1}(1), X_{2}(1) ; y\right) ;
$$

3) $\exists i \in\left[M_{1}\right] \backslash\{1\}, j \in\left[M_{2}\right] \backslash\{1\}$, s.t.

$$
i\left(X_{1}(i), X_{2}(j) ; y\right) \geq i\left(X_{1}(1), X_{2}(1) ; y\right) .
$$

Therefore, $\mathbb{E}\left[\epsilon_{1,1}\right]$ can be bounded from above as

$$
\begin{array}{r}
\mathbb{E}\left[\epsilon_{1,1}\right] \\
\leq \operatorname{Pr}\left[\left\{\bigcup_{i=2}^{M_{1}}\left\{i\left(X_{1}(i), X_{2}(1) ; Y\right) \geq i\left(X_{1}(1), X_{2}(1) ; Y\right)\right\}\right\}\right. \\
\cup\left\{\bigcup_{j=2}^{M_{2}}\left\{i\left(X_{1}(1), X_{2}(j) ; Y\right) \geq i\left(X_{1}(1), X_{2}(1) ; Y\right)\right\}\right\}
\end{array}
$$




$$
\begin{aligned}
& \left.\cup\left\{\bigcup_{\substack{i \in\left[M_{1}\right] \backslash\{1\} \\
j \in\left[M_{2} \backslash \backslash\{1\}\right.}}\left\{i\left(X_{1}(i), X_{2}(j) ; Y\right) \geq i\left(X_{1}(1), X_{2}(1) ; Y\right)\right\}\right\}\right] \\
& =\operatorname{Pr}\left[\left\{\bigcup_{(125)}^{M_{1}}\left\{i\left(X_{1}(i) ; Y \mid X_{2}(1)\right) \geq i\left(X_{1}(1) ; Y \mid X_{2}(1)\right)\right\}\right\}\right. \\
& \cup\left\{\bigcup_{i=2}^{M_{2}}\left\{i\left(X_{2}(j) ; Y \mid X_{1}(1)\right) \geq i\left(X_{2}(1) ; Y \mid X_{1}(1)\right)\right\}\right\} \\
& \cup\left\{\bigcup_{\substack{i \in\left[M_{1}\right] \backslash\{1\} \\
j \in\left[M_{2}\right] \backslash\{1\}}}^{\bigcup}\left\{i\left(X_{1}(i), X_{2}(j) ; Y\right) \geq i\left(X_{1}(1), X_{2}(1) ; Y\right)\right\}\right\},
\end{aligned}
$$

where 125 is an inequality instead of an equality since the decoder might resolve some ties correctly, and (126) removes common terms from the first two terms of (125), thereby replacing information density by conditional information density.

Let $W=\left(X_{1}(1), X_{2}(1), Y\right)$. Then

$$
\begin{aligned}
& \mathbb{E}\left[\epsilon_{1,1}\right] \\
& =\mathbb{E}[\operatorname{Pr}[ \\
& \left\{\bigcup_{i=2}^{M_{1}}\left\{i\left(X_{1}(i) ; Y \mid X_{2}(1)\right) \geq i\left(X_{1}(1) ; Y \mid X_{2}(1)\right)\right\}\right\} \\
& \left\{\bigcup_{j=2}^{M_{2}}\left\{i\left(X_{2}(j) ; Y \mid X_{1}(1)\right) \geq i\left(X_{2}(1) ; Y \mid X_{1}(1)\right)\right\}\right\} \cup \\
& \left\{\begin{array}{l}
\left.\left.\bigcup_{i \in\left[M_{1}\right] \backslash\{1\}}\left\{i\left(X_{1}(i), X_{2}(j) ; Y\right) \geq i\left(X_{1}(1), X_{2}(1) ; Y\right)\right\}\right\} \mid T\right] \\
j \in\left[M_{2}\right] \backslash\{1\}
\end{array}\right. \\
& \leq \mathbb{E}[\min \{1, \\
& \quad\left(M_{1}-1\right) \operatorname{Pr}\left[\left\{i\left(\bar{X}_{1} ; Y \mid X_{2}\right) \geq i\left(X_{1} ; Y \mid X_{2}\right)\right\} \mid T\right] \\
& \quad+\left(M_{2}-1\right) \operatorname{Pr}\left[\left\{i\left(\bar{X}_{2} ; Y \mid X_{1}\right) \geq i\left(X_{2} ; Y \mid X_{1}\right)\right\} \mid T\right] \\
& \quad+\left(M_{1}-1\right)\left(M_{2}-1\right) \\
& \left.\left.\quad \quad \operatorname{Pr}\left[\left\{i\left(\bar{X}_{1}, \bar{X}_{2} ; Y\right) \geq i\left(X_{1}, X_{2} ; Y\right)\right\} \mid T\right]\right\}\right],
\end{aligned}
$$

where (127) follows from the law of iterated expectation, and (128) holds by the bounded nature of probability and symmetry of our code design.

Remark 12: The authors in [24] achieve lower decoder complexity in the symmetrical rate case by replacing the three events in (124) by one. While we do not assume the symmetrical rate point, we note that only events corresponding to constraints that are active at a given rate point have a nonnegligible impact in 128. This observation enables decoder simplification for most rate points.

Prior to stating the achievability theorem, we generalize the inverse complementary $\operatorname{CDF} Q^{-1}(\cdot)$ to higher dimension. Let
$Z$ be a Gaussian random vector in $\mathbb{R}^{d}$ with mean zero and covariance matrix $\boldsymbol{K}_{\boldsymbol{Z} Z}$, denote the set $Q_{\text {inv }}\left(\boldsymbol{K}_{\boldsymbol{Z} \boldsymbol{Z}}, \epsilon\right)$ as

$$
Q_{\mathrm{inv}}\left(\boldsymbol{K}_{\boldsymbol{Z} \boldsymbol{Z}}, \epsilon\right) \triangleq\left\{\boldsymbol{z} \in \mathbb{R}^{d}: \operatorname{Pr}[\boldsymbol{Z} \leq \boldsymbol{z}] \geq 1-\epsilon\right\} .
$$

Theorem 14: (Random coding finite-blocklength bound and third-order achievability bound on the DM-2-MAC). Consider a DM-2-MAC $\left(\mathcal{X}_{1} \times \mathcal{X}_{2}, P_{Y \mid X_{1}, X_{2}}, \mathcal{Y}\right)$. Let each symbol of each codeword for transmitter $i$ be drawn i.i.d. according to $P_{X_{i}}$, for $i \in\{1,2\}$. Then there exists an $\left(n, M_{1}, M_{2}, \epsilon\right)$ code such that for any blocklength $n$

$$
\epsilon \leq \mathbb{E}\left[\min \left\{1, E_{1}+E_{2}+E_{12}\right\}\right],
$$

and for large enough blocklength $n$

$$
\overline{\boldsymbol{R}} \in \overline{\boldsymbol{I}}-\frac{Q_{\text {inv }}(\mathrm{V}, \epsilon)}{\sqrt{n}}+\frac{\log n}{2 n} \mathbf{1}-O\left(\frac{1}{n}\right) \mathbf{1},
$$

providing the following moment assumptions are satisfied

$$
\begin{aligned}
V^{Y}\left(P_{X_{1}} \mid P_{X_{2}}\right) & >0, & V^{Y}\left(P_{X_{2}} \mid P_{X_{1}}\right) & >0, \\
V^{Y}\left(P_{X_{1}}, P_{X_{2}}\right) & >0, & T\left(P_{X_{1}} \mid P_{X_{2}}\right) & <\infty, \\
T\left(P_{X_{2}} \mid P_{X_{1}}\right) & <\infty, & T\left(P_{X_{1}}, P_{X_{2}}\right) & <\infty,
\end{aligned}
$$

where

$$
\begin{aligned}
& F_{1} \triangleq 2\left(\frac{\log 2}{\sqrt{2 \pi V\left(P_{X_{1}} \mid P_{X_{2}}\right)}}+2 \frac{C_{0} T\left(P_{X_{1}} \mid P_{X_{2}}\right)}{V\left(P_{X_{1}} \mid P_{X_{2}}\right)^{3 / 2}}\right) \\
& F_{2} \triangleq 2\left(\frac{\log 2}{\sqrt{2 \pi V\left(P_{X_{2}} \mid P_{X_{1}}\right)}}+2 \frac{C_{0} T\left(P_{X_{2}} \mid P_{X_{1}}\right)}{V\left(P_{X_{2}} \mid P_{X_{1}}\right)^{3 / 2}}\right) \\
& F_{12} \triangleq 2\left(\frac{\log 2}{\sqrt{2 \pi V\left(P_{X_{1}}, P_{X_{2}}\right)}}+2 \frac{C_{0} T\left(P_{X_{1}}, P_{X_{2}}\right)}{V\left(P_{X_{1}}, P_{X_{2}}\right)^{3 / 2}}\right) \\
& E_{1} \triangleq M_{1} \frac{F_{1}}{\sqrt{n}} \exp \left(-i\left(X_{1}^{n} ; Y^{n} \mid X_{2}^{n}\right)\right) \\
& E_{2} \triangleq M_{2} \frac{F_{2}}{\sqrt{n}} \exp \left(-i\left(X_{2 j} ; Y_{j} \mid X_{1 j}\right)\right) \\
& E_{12} \triangleq M_{1} M_{2} \frac{F_{12}}{\sqrt{n}} \exp \left(-i\left(X_{1}^{n}, X_{2}^{n} ; Y^{n}\right)\right) \\
& \overline{\boldsymbol{R}} \triangleq\left[\begin{array}{c}
R_{1} \\
R_{2} \\
R_{1}+R_{2}
\end{array}\right], \overline{\boldsymbol{I}} \triangleq\left[\begin{array}{l}
\mathbb{E}\left[i\left(X_{1} ; Y \mid X_{2}\right)\right] \\
\mathbb{E}\left[i\left(X_{2} ; Y \mid X_{1}\right)\right] \\
\mathbb{E}\left[i\left(X_{1}, X_{2} ; Y\right)\right]
\end{array}\right],
\end{aligned}
$$

$\mathrm{V}$ is the covariance matrix of

$$
\overline{\boldsymbol{i}}\left(P_{X_{1}}, P_{X_{2}}\right) \triangleq\left[\begin{array}{l}
i\left(X_{1} ; Y \mid X_{2}\right) \\
i\left(X_{2} ; Y \mid X_{1}\right) \\
i\left(X_{1}, X_{2} ; Y\right)
\end{array}\right],
$$

and $Q_{\text {inv }}$ is defined in 129 .

The proof of Theorem 14 requires a multi-dimensional version of Berry Esséen theorem, shown as Lemma 2 below.

Lemma 2: (Multi-dimensional Berry-Esséen Theorem, [27] Lemma 15], [20. Cor. 8]). Let $\boldsymbol{U}_{1}, \ldots, \boldsymbol{U}_{n} \in \mathbb{R}^{d}$ be a sequence of i.i.d. random vectors with mean zero and covariance matrix $\boldsymbol{\Sigma}$ of rank $r \triangleq \operatorname{rank}(\boldsymbol{\Sigma})$. Let $\boldsymbol{Z} \in \mathbb{R}^{d}$ be a Gaussian vector with mean zero and the same covariance matrix $\Sigma$. Let $\mathrm{T}$ be a $d \times r$ matrix, where the columns of $\mathrm{T}$ are the $r$ normalized eigenvectors of $\boldsymbol{\Sigma}$ with non-zero eigenvalues. Define $\boldsymbol{W}_{1}, \ldots, \boldsymbol{W}_{n} \in \mathbb{R}^{r}$ to be a sequence of i.i.d. random 
vectors, such that $\boldsymbol{U}_{i}=\mathrm{T} \boldsymbol{W}_{i}$ for all $i \in[n]$. If $r \geq 1$, then for all $n$,

$$
\sup _{\boldsymbol{z} \in \mathbb{R}^{d}}\left|\operatorname{Pr}\left[\frac{1}{\sqrt{n}} \sum_{j=1}^{n} \boldsymbol{U}_{i} \leq \boldsymbol{z}\right]-\operatorname{Pr}[\boldsymbol{Z} \leq \boldsymbol{z}]\right| \leq \frac{400 d^{\frac{1}{4}} \beta_{r}}{\lambda_{\min }^{\frac{3}{2}}} \frac{1}{\sqrt{n}},
$$

where $\boldsymbol{\Sigma}_{r}$ is the covariance matrix of $\boldsymbol{W}_{1}, \beta_{r} \triangleq \mathbb{E}\left[\left\|\boldsymbol{W}_{1}\right\|_{2}^{3}\right]$ $\left(\|\cdot\|\right.$ is the $\ell^{2}$ norm), and $\lambda_{\min }$ is the minimum eigenvalue of $\boldsymbol{\Sigma}_{r}$.

Proof of Theorem 14. Setting $X_{1}=X_{1}^{n}, \bar{X}_{1}=\bar{X}_{1}^{n}, X_{2}=$ $X_{2}^{n}, \bar{X}_{2}=\bar{X}_{2}^{n}, Y=Y^{n}$ in Theorem 13 , we note that there exists an $\left(n, M_{1}, M_{2}, \epsilon^{\prime}\right)$ code with

$$
\epsilon^{\prime} \leq \mathbb{E}\left[\min \left\{1, V_{1}+V_{2}+V_{12}\right\}\right],
$$

where

$$
\begin{aligned}
& \begin{aligned}
V_{1}= & \left(M_{1}-1\right) \\
& \quad \operatorname{Pr}\left[i\left(\bar{X}_{1}^{n} ; Y^{n} \mid X_{2}^{n}\right) \geq i\left(X_{1}^{n} ; Y^{n} \mid X_{2}^{n}\right) \mid X_{1}^{n}, X_{2}^{n}, Y^{n}\right], \\
V_{2}= & \left(M_{2}-1\right) \\
& \quad \operatorname{Pr}\left[i\left(\bar{X}_{2}^{n} ; Y^{n} \mid X_{1}^{n}\right) \geq i\left(X_{2}^{n} ; Y^{n} \mid X_{1}^{n}\right) \mid X_{1}^{n}, X_{2}^{n}, Y^{n}\right],
\end{aligned}
\end{aligned}
$$

$$
\begin{aligned}
V_{12}= & \left(M_{1}-1\right)\left(M_{2}-1\right) \\
& \left.\operatorname{Pr}\left[i\left(\bar{X}_{1}^{n}, \bar{X}_{2}^{n} ; Y^{n}\right) \geq i\left(X_{1}^{n}, X_{2}^{n} ; Y\right)^{n} \mid X_{1}^{n}, X_{2}^{n}, Y^{n}\right]\right\},
\end{aligned}
$$

and

$$
\begin{aligned}
& P_{X_{1}^{n} X_{2}^{n} \bar{X}_{1}^{n} \bar{X}_{2}^{n} Y^{n}}\left(x_{1}^{n}, x_{2}^{n}, \bar{x}_{1}^{n}, \bar{x}_{2}^{n}, y^{n}\right) \\
& =P_{X_{1}^{n} \bar{X}_{1}^{n}}\left(x_{1}^{n}, \bar{x}_{1}^{n}\right) P_{X_{2}^{n} \bar{X}_{2}^{n}}\left(x_{2}^{n}, \bar{x}_{2}^{n}\right) P_{Y^{n} \mid X_{1}^{n} X_{2}^{n}}\left(y^{n} \mid x_{1}^{n}, x_{2}^{n}\right) \\
& =P_{X_{1}^{n}}\left(x_{1}^{n}\right) P_{X_{1}^{n}}\left(\bar{x}_{1}^{n}\right) P_{X_{2}^{n}}\left(x_{2}^{n}\right) P_{X_{2}^{n}}\left(\bar{x}_{2}^{n}\right) P_{Y^{n} \mid X^{n}}\left(y^{n} \mid x^{n}\right),
\end{aligned}
$$

as the codewords for transmitter $i \in\{1,2\}$ are drawn i.i.d. according to $P_{X_{i}^{n}}=P_{X_{i}}^{n}$.

Denote for brevity

$$
\begin{aligned}
I_{1 n} \triangleq i\left(X_{1}^{n} ; Y^{n} \mid X_{2}^{n}\right) & =\sum_{j=1}^{n} i\left(X_{1 j} ; Y_{j} \mid X_{2 j}\right), \\
I_{2 n} \triangleq i\left(X_{2}^{n} ; Y^{n} \mid X_{1}^{n}\right) & =\sum_{j=1}^{n} i\left(X_{2 j} ; Y_{j} \mid X_{1 j}\right), \\
I_{n} \triangleq i\left(X_{1}^{n}, X_{2}^{n} ; Y^{n}\right) & =\sum_{j=1}^{n} i\left(X_{1 j}, X_{2 j} ; Y_{j}\right), \\
\bar{I}_{1 n} \triangleq i\left(\bar{X}_{1}^{n} ; Y^{n} \mid X_{2}^{n}\right) & =\sum_{j=1}^{n} i\left(\bar{X}_{1 j} ; Y_{j} \mid X_{2 j}\right), \\
\bar{I}_{2 n} \triangleq i\left(\bar{X}_{2}^{n} ; Y^{n} \mid X_{1}^{n}\right) & =\sum_{j=1}^{n} i\left(\bar{X}_{2 j} ; Y_{j} \mid X_{1 j}\right), \\
\bar{I}_{n} \triangleq i\left(\bar{X}_{1}^{n}, \bar{X}_{2}^{n} ; Y^{n}\right) & =\sum_{j=1}^{n} i\left(\bar{X}_{1 j}, \bar{X}_{2 j} ; Y_{j}\right),
\end{aligned}
$$

where $\left(X_{1 j}, X_{2 j}\right)$ and $\left(\bar{X}_{1 j}, \bar{X}_{2 j}\right)$ are the $j$-th symbols of the transmitted codeword pair and an untransmitted codeword pair, respectively.
Note that since the codewords are drawn i.i.d. by assumption, $\bar{X}_{1}^{n}$ is independent of $X_{1}^{n}, X_{2}^{n}$, and $Y^{n}$. If $P_{Y^{n} \mid X_{1}^{n}, X_{2}^{n}}\left(Y^{n} \mid \bar{x}_{1}^{n}, X_{2}^{n}\right)>0$, then

$$
\begin{aligned}
& \operatorname{Pr}\left[\bar{X}_{1}^{n}=\bar{x}_{1}^{n} \mid X_{1}^{n}, X_{2}^{n}, Y^{n}\right] \\
& =\operatorname{Pr}\left[\bar{X}_{1}^{n}=\bar{x}_{1}^{n} \mid X_{2}^{n}\right] \\
& =\operatorname{Pr}\left[\bar{X}_{1}^{n}=\bar{x}_{1}^{n} \mid X_{2}^{n}\right] \frac{P_{Y^{n} \mid X_{1}^{n}, X_{2}^{n}}\left(Y^{n} \mid \bar{x}_{1}^{n}, X_{2}^{n}\right)}{P_{Y^{n} \mid X_{2}^{n}}\left(Y^{n} \mid X_{2}^{n}\right)} \\
& \cdot \frac{P_{Y^{n} \mid X_{2}^{n}\left(Y^{n} \mid X_{2}^{n}\right)}}{P_{Y^{n}} \mid X_{1}^{n}, X_{2}^{n}\left(Y^{n} \mid \bar{x}_{1}^{n}, X_{2}^{n}\right)} \\
& =\operatorname{Pr}\left[X_{1}^{n}=\bar{x}_{1}^{n} \mid Y^{n}, X_{2}^{n}\right] \exp \left\{-i\left(\bar{x}_{1}^{n} ; Y^{n} \mid X_{2}^{n}\right)\right\} .
\end{aligned}
$$

If $P_{Y^{n} \mid X_{1}^{n}, X_{2}^{n}}\left(Y^{n} \mid \bar{x}_{1}^{n}, X_{2}^{n}\right)=0$, then we stop at 156. Note that the following derivation only sums over $\bar{x}_{1}^{n}$ such that $P_{Y^{n} \mid X_{1}^{n}, X_{2}^{n}}\left(Y^{n} \mid \bar{x}_{1}^{n}, X_{2}^{n}\right)>0$ as $P_{Y^{n} \mid X_{1}^{n}, X_{2}^{n}}\left(Y^{n} \mid \bar{x}_{1}^{n}, X_{2}^{n}\right)=0$ implies $\bar{I}_{1 n}=-\infty$.

Summing over all $\bar{x}_{1}^{n}$ such that $\bar{I}_{1 n} \geq \zeta$ gives

$$
\begin{aligned}
\operatorname{Pr}\left[\bar{I}_{1 n} \geq \zeta \mid Y^{n}, X_{2}^{n}\right] & =\mathbb{E}\left[\exp \left\{-I_{1 n}\right\} \mathbb{1}\left\{I_{1 n} \geq \zeta\right\} \mid Y^{n}, X_{2}^{n}\right] \\
& \leq \frac{F_{1}}{\sqrt{n}} \exp (-\zeta),
\end{aligned}
$$

where (158) follows from Lemma 1

Plugging (158) into (145), we obtain

$$
V_{1} \leq M_{1} \frac{F_{1}}{\sqrt{n}} \exp \left(-I_{1 n}\right)=E_{1} .
$$

A similar approach yields

$$
\begin{aligned}
V_{2} & \leq M_{2} \frac{F_{2}}{\sqrt{n}} \exp \left(-I_{2 n}\right)=E_{2}, \\
V_{12} & \leq M_{1} M_{2} \frac{F_{12}}{\sqrt{n}} \exp \left(-I_{n}\right)=E_{12} .
\end{aligned}
$$

Therefore

$$
\begin{aligned}
\epsilon^{\prime} \leq & \mathbb{E}\left[\min \left\{1, E_{1}+E_{2}+E_{12}\right\}\right] \\
= & \operatorname{Pr}\left[E_{1}+E_{2}+E_{12}>1\right] \\
& +\mathbb{E}\left[\left(E_{1}+E_{2}+E_{12}\right) \mathbb{1}\left\{\left(E_{1}+E_{2}+E_{12}\right) \leq 1\right\}\right] \\
\leq & \operatorname{Pr}\left[E_{1}+E_{2}+E_{12}>1\right]+\mathbb{E}\left[E_{1} \mathbb{1}\left\{E_{1} \leq 1\right\}\right] \\
& +\mathbb{E}\left[E_{2} \mathbb{1}\left\{E_{2} \leq 1\right\}\right]+\mathbb{E}\left[E_{12} \mathbb{1}\left\{E_{12} \leq 1\right\}\right] \\
\leq & \operatorname{Pr}\left[E_{1}+E_{2}+E_{12}>1\right]+\frac{F_{1}}{\sqrt{n}}+\frac{F_{2}}{\sqrt{n}}+\frac{F_{12}}{\sqrt{n}} \\
= & 1-\operatorname{Pr}\left[E_{1}+E_{2}+E_{12} \leq 1\right]+\frac{F_{1}}{\sqrt{n}}+\frac{F_{2}}{\sqrt{n}}+\frac{F_{12}}{\sqrt{n}} \\
\leq & 1-\operatorname{Pr}\left[\left\{E_{1} \leq \frac{1}{3}\right\} \cap\left\{E_{2} \leq \frac{1}{3}\right\} \cap\left\{E_{12} \leq \frac{1}{3}\right\}\right] \\
& +\frac{F_{1}}{\sqrt{n}}+\frac{F_{2}}{\sqrt{n}}+\frac{F_{12}}{\sqrt{n}},
\end{aligned}
$$

where 163 holds by separating the cases based on whether $E_{1}+E_{2}+E_{12}<1$ or not, (164) follows from linearity of expectation and weakening the indicator function threshold, applying Lemma 1 to the each of the last three terms in (164) yields (165), and (167) holds since the event $\left\{\left\{E_{1} \leq \frac{1}{3}\right\} \cap\right.$ $\left.\left\{E_{2} \leq \frac{1}{3}\right\} \cap\left\{E_{12} \leq \frac{1}{3}\right\}\right\}$ is a subset of the event $\left\{E_{1}+E_{2}+\right.$ $\left.E_{12} \leq 1\right\}$. 
Denote

$$
\begin{aligned}
& \boldsymbol{U}_{j} \triangleq\left[\begin{array}{l}
i\left(X_{1 j} ; Y_{j} \mid X_{2 j}\right) \\
i\left(X_{2 j} ; Y_{j} \mid X_{1 j}\right) \\
i\left(X_{1 j}, X_{2 j} ; Y_{j}\right)
\end{array}\right]-\overline{\boldsymbol{I}}, \forall j \in[n], \\
& \boldsymbol{S}_{n} \triangleq \frac{1}{\sqrt{n}} \sum_{j=1}^{n} \boldsymbol{U}_{j}=\frac{1}{\sqrt{n}}\left[\begin{array}{c}
I_{1 n} \\
I_{2 n} \\
I_{n}
\end{array}\right]-\sqrt{n \overline{\boldsymbol{I}}},
\end{aligned}
$$

where each $\boldsymbol{U}_{j}, j \in[n]$, is a random vector with mean zero and covariance matrix $\mathrm{V}$. Note $\mathbb{E}\left[\left\|\boldsymbol{U}_{1}\right\|_{2}^{3}\right]$ is finite by the moment assumptions (133)-(134); hence Lemma 2 is applicable.

Therefore,

$$
\begin{aligned}
& \operatorname{Pr}\left[\left\{E_{1} \leq \frac{1}{3}\right\} \cap\left\{E_{2} \leq \frac{1}{3}\right\} \cap\left\{E_{12} \leq \frac{1}{3}\right\}\right] \\
&=\operatorname{Pr} {\left[\left\{I_{1 n} \geq \log M_{1}+\log 3 F_{1}-\frac{1}{2} \log n\right\} \cap\right.} \\
&\left\{I_{2 n} \geq \log M_{2}+\log 3 F_{2}-\frac{1}{2} \log n\right\} \cap \\
&\left.\left\{I_{n} \geq \log M_{1}+\log M_{2}+\log 3 F_{12}-\frac{1}{2} \log n\right\}\right] \\
&=\operatorname{Pr} {\left[\boldsymbol{S}_{n} \geq \sqrt{n}\left(\overline{\boldsymbol{R}}-\overline{\boldsymbol{I}}-\frac{\log n}{2 n} \mathbf{1}+O\left(\frac{1}{n}\right) \mathbf{1}\right)\right] } \\
&=1- \operatorname{Pr}\left[\boldsymbol{S}_{n}<\sqrt{n}\left(\overline{\boldsymbol{R}}-\overline{\boldsymbol{I}}-\frac{\log n}{2 n} \mathbf{1}+O\left(\frac{1}{n}\right) \mathbf{1}\right)\right] \\
& \geq 1-\operatorname{Pr}\left[\boldsymbol{S}_{n} \leq \sqrt{n}\left(\overline{\boldsymbol{R}}-\overline{\boldsymbol{I}}-\frac{\log n}{2 n} \mathbf{1}+O\left(\frac{1}{n}\right) \mathbf{1}\right)\right] \\
& \geq 1-\operatorname{Pr}\left[\boldsymbol{Z} \leq \sqrt{n}\left(\overline{\boldsymbol{R}}-\overline{\boldsymbol{I}}-\frac{\log n}{2 n} \mathbf{1}+O\left(\frac{1}{n}\right) \mathbf{1}\right)\right]
\end{aligned}
$$

where (170) follows by expanding $E_{1}, E_{2}$, and $E_{12}$ using (138)-(140), (171) rewrites (170) using the definition of $\boldsymbol{S}_{n}, \overline{\boldsymbol{R}}$ and $\overline{\boldsymbol{I}}$, and (174) follows from the multi-dimensional BerryEsséen Theorem, Lemma 2

For any rate $\left(R_{1}, R_{2}\right)$ satisfying

$$
\overline{\boldsymbol{R}} \in \overline{\boldsymbol{I}}-\frac{Q_{\text {inv }}\left(\mathrm{V}, \epsilon-\frac{c}{\sqrt{n}}\right)}{\sqrt{n}}+\frac{\log n}{2 n} \mathbf{1}-O\left(\frac{1}{n}\right) \mathbf{1},
$$

by the definition of $Q_{\text {inv }}$ in 129 , we have

$$
\operatorname{Pr}\left[\boldsymbol{Z} \leq \sqrt{n}\left(\overline{\boldsymbol{R}}-\overline{\boldsymbol{I}}-\frac{\log n}{2 n} \mathbf{1}+O\left(\frac{1}{n}\right) \mathbf{1}\right)\right] \leq \epsilon-\frac{c}{\sqrt{n}} .
$$

Therefore, (174) becomes

$$
\begin{aligned}
& \operatorname{Pr}\left[\left\{E_{1} \leq \frac{1}{3}\right\} \cap\left\{E_{2} \leq \frac{1}{3}\right\} \cap\left\{E_{12} \leq \frac{1}{3}\right\}\right] \\
\geq & 1-\epsilon+\frac{c}{\sqrt{n}}-O\left(\frac{1}{\sqrt{n}}\right) .
\end{aligned}
$$

Substituting (177) into (167) gives

$$
\begin{aligned}
\epsilon^{\prime} & \leq 1-\left(1-\epsilon+\frac{c}{\sqrt{n}}-O\left(\frac{1}{\sqrt{n}}\right)\right)+\frac{F_{1}+F_{2}+F_{12}}{\sqrt{n}} \\
& =\epsilon+\frac{F_{1}+F_{2}+F_{12}-c}{\sqrt{n}}+O\left(\frac{1}{\sqrt{n}}\right) .
\end{aligned}
$$

Recall that the constants $F_{1}, F_{2}$, and $F_{12}$ are positive and finite by the moment assumptions (132)-(134). Therefore, there exists some constant $c$ and $N$ such that $\epsilon^{\prime} \leq \epsilon$ for all $n \geq N$. Finally, we apply part 1) of [27, Lemma 16] to conclude the existence of an $\left(n, M_{1}, M_{2}, \epsilon\right)$ code when

$$
\overline{\boldsymbol{R}} \in \overline{\boldsymbol{I}}-\frac{Q_{\mathrm{inv}}(\mathrm{V}, \epsilon)}{\sqrt{n}}+\frac{\log n}{2 n} \mathbf{1}-O\left(\frac{1}{n}\right) \mathbf{1} .
$$

\section{RCU BOUNDS FOR LDPC CODES}

\section{A. RCU Bound for LDPC Code on the DM-PPC}

In this section, we apply the generalized RCU bound, Theorem 10, to the $\operatorname{LDPC}(\lambda, \rho, \delta ; n)$ ensemble to prove an achievability result for LDPC codes. The LDPC achievability result matches the optimal achievable performance of an unrestricted point-to-point code in its first- and second-order terms. The penalty incurred for using the LDPC code ensemble is $\frac{\log \alpha}{n}$, where $\alpha=\left.\alpha_{1}\right|_{\left(\lambda_{1}, \rho_{1}\right)=(\lambda, \rho)}$ and $\alpha_{1}$ is from 36. We show that $\frac{\log \alpha}{n}$ is $O\left(\frac{\log n}{n}\right)$ if $\rho=\kappa n$ and $\kappa$ approaches zero no more quickly than $\Theta\left(\frac{\log n}{n}\right)$, provided that we first expurgate codes with low minimal distance, as shown in Appendix $D$ Whether the penalty in the third-order term results from the LDPC structure or the bounding technique remains an open problem.

The PPC and MAC achievability results for i.i.d. $P_{X}$ codes (see Theorem 11 and Theorem 14 do not apply for the LDPC code ensemble. The challenges in applying the proof techniques in Theorem 11 and Theorem 14 to LDPC codes are as follows.

1) The codewords in our LDPC code ensembles, $\operatorname{LDPC}(\lambda, \rho ; n)$ and $\operatorname{LDPC}(\lambda, \rho, \delta ; n)$, are not independent of each other. For example, if a particular vector is known to be in a random codebook, then it must be true that the underlying Tanner graph describes a family of parity-check equations that are consistent with the given codeword. Further, all other codewords in the codebook must satisfy the same parity checks. Thus, both the parity-check matrix and the other codewords are dependent on the given codeword. For example, when $q=2$ and the check node degree $\rho$ is odd, if $x^{n}$ is a codeword, then $x^{n}+1^{n}$ cannot be a codeword, and vice versa.

2) The symbols within a codeword for the $\operatorname{LDPC}(\lambda, \rho ; n)$ ensemble are not independent. In fact, the symbols within a codeword must be dependent to fulfill the set of parity-check equations.

Nonetheless, the code design of the $\operatorname{LDPC}(\lambda, \rho, \delta ; n)$ ensemble meets the condition of our generalized RCU bound, Theorem 10 . 
We here present two results for the $\operatorname{LDPC}(\lambda, \rho, \delta ; n)$ ensemble. The first one is a finite-blocklength error probability bound, which holds for any blocklength $n$. The second one is an asymptotic achievability expansion.

Theorem 15: (LDPC code finite-blocklength bound and second-order-optimal achievability for the DM-PPC). Consider a DM-PPC with channel transition probability $P_{Y \mid X}$ and rational input distribution $P_{X}$, chosen to approximate the optimal input distribution $P_{X}^{*}$. Then there exist LDPC parameters $(\lambda, \rho)$ for which the $\operatorname{LDPC}(\lambda, \rho, \delta ; n)$ ensemble, with $\delta(\cdot)$ chosen to approximate $P_{X}$, contains at least one code with average error probability less than $\epsilon$ such that for any blocklength $n$

$$
\epsilon \leq \mathbb{E}\left[\min \left\{1, \alpha M \frac{A\left(P_{X}\right)}{\sqrt{n}} \exp \left(-I_{n}\right)\right\}\right],
$$

and for large enough blocklength $n$

$$
\begin{aligned}
& R=1-\frac{\lambda}{\rho}=\frac{\log M}{n} \geq C\left(P_{X}\right) \\
& -\sqrt{\frac{V\left(P_{X}\right)}{n}} Q^{-1}(\epsilon)+\frac{\log n}{2 n}-\frac{\log \alpha}{n}-O\left(\frac{1}{n}\right),
\end{aligned}
$$

providing the following moment assumptions are satisfied when $X \sim P_{X}$

$$
\begin{aligned}
I\left(P_{X}\right) & >0, \\
V^{Y}\left(P_{X}\right) & >0, \\
T\left(P_{X}\right) & <\infty .
\end{aligned}
$$

Here

$$
\begin{aligned}
A\left(P_{X}\right) & =2\left(\frac{\log 2}{\sqrt{2 \pi V\left(P_{X}\right)}}+2 \frac{C_{0} T\left(P_{X}\right)}{V\left(P_{X}\right)^{3 / 2}}\right), \\
\alpha & =\max _{\boldsymbol{t} \in \mathcal{T}_{q}^{n} \backslash\left\{\mathcal{T}_{q}^{n}(\mathbf{0})\right\}} \frac{\bar{S}^{n}(\boldsymbol{t})}{(M-1) B(n, \boldsymbol{t}) q^{-n}},
\end{aligned}
$$

$\mathcal{T}_{q}^{n}$ is the set of all possibles types for a list of $n$ elements in $\operatorname{GF}(q), \mathcal{T}_{q}^{n}(\mathbf{0})$ is the type of the all-zero vector, $B(n, \boldsymbol{t})$ is the number of length- $n$ vectors with type $t$ (the multinomial coefficient), $M=q^{n R}, \bar{S}^{n}(\boldsymbol{t})$ is the $\operatorname{LDPC}(\lambda, \rho ; n)$ ensembleaverage number of type- $t$ vectors, and $C\left(P_{X}\right)$ is the mutual information $i\left(X^{n} ; Y^{n}\right)$ evaluated at input distribution $P_{X}$.

Remark 13: Due to the nature of the quantizer $\delta(\cdot)$, we are only able to achieve rational input distributions that are integer multiples of $\frac{1}{q}$. When the optimal input distribution $P_{X}^{*}$ is irrational or not an integer multiple of $\frac{1}{q}$, then a large alphabet size $q$ may be required to closely approximate $P_{X}^{*}$.

Proof of Theorem 15. Since the codeword distribution under the LDPC design meets the constraint of Theorem 10, the generalized RCU bound is applicable. Setting $X=X^{n}, \bar{X}=$ $\bar{X}^{n}, Y=Y^{n}$ in Theorem 10. we note that there exists at least one code in this ensemble with average error probability $\epsilon^{\prime}$ satisfying

$$
\epsilon^{\prime} \leq \mathbb{E}\left[\min \left\{1, M \operatorname{Pr}\left[i\left(\bar{X}^{n} ; Y^{n}\right) \geq i\left(X^{n} ; Y^{n}\right) \mid X^{n}, Y^{n}\right]\right\}\right],
$$

where

$$
P_{X^{n} \bar{X}^{n} Y^{n}}\left(x^{n}, \bar{x}^{n}, y^{n}\right)=P_{X^{n}, \bar{X}^{n}}\left(x^{n}, \bar{x}^{n}\right) P_{Y^{n} \mid X^{n}}\left(y^{n} \mid x^{n}\right) .
$$

Here $P_{X^{n}, \bar{X}^{n}}\left(x^{n}, \bar{x}^{n}\right) \neq P_{X^{n}}\left(x^{n}\right) P_{X^{n}}\left(\bar{x}^{n}\right)$ in general due to codeword dependence in the $\operatorname{LDPC}(\lambda, \rho, \delta ; n)$ ensemble.

For the $\operatorname{LDPC}(\lambda, \rho, \delta ; n)$ ensemble, $Y^{n}$ depends on $\bar{X}^{n}$ only through its dependence on $X^{n}$ and therefore $\bar{X}^{n} \rightarrow$ $X^{n} \rightarrow Y^{n}$ forms a Markov chain. Thus,

$$
\begin{aligned}
\operatorname{Pr}\left[\bar{X}^{n}=\bar{x}^{n} \mid X^{n}, Y^{n}\right] & =\operatorname{Pr}\left[\bar{X}^{n}=\bar{x}^{n} \mid X^{n}\right] \\
& =\frac{\operatorname{Pr}\left[\bar{X}^{n}=\bar{x}^{n}, X^{n}=x^{n}\right]}{\operatorname{Pr}\left[X^{n}=x^{n}\right]} .
\end{aligned}
$$

Recall from Appendix A equation 231) that

$$
\operatorname{Pr}\left[\boldsymbol{C}_{1}+\boldsymbol{v}=\boldsymbol{a}, C_{m^{\prime}}+\boldsymbol{v}=\boldsymbol{a}^{\prime}\right] \leq q^{-n} \alpha q^{-n},
$$

where $C_{1}$ is the first codeword in a random LDPC codebook $\boldsymbol{C}$ from the $\operatorname{LDPC}(\lambda, \rho ; n)$ ensemble, $\boldsymbol{C}_{m^{\prime}}, m^{\prime} \neq 1$, is another codeword in $\boldsymbol{C}$, and $\boldsymbol{v}$ is the random coset vector. Thus

$$
\begin{aligned}
& \operatorname{Pr}\left[\bar{X}^{n}=\bar{x}^{n}, X^{n}=x^{n}\right] \\
& =\sum_{a, a^{\prime}: \delta(a)=x^{n}, \delta\left(a^{\prime}\right)=\bar{x}^{n}} \operatorname{Pr}\left[\boldsymbol{C}_{1}+\boldsymbol{v}=\boldsymbol{a}, \boldsymbol{C}_{m^{\prime}}+\boldsymbol{v}=\boldsymbol{a}^{\prime}\right] \\
& \leq \sum_{a, a^{\prime}: \delta(a)=x^{n}, \delta\left(a^{\prime}\right)=\bar{x}^{n}} q^{-n} \alpha q^{-n} \\
& =\alpha \sum_{a: \delta(a)=x^{n}} q^{-n} \cdot \sum_{a: \delta\left(a^{\prime}\right)=\bar{x}^{n}} q^{-n} \\
& =\alpha \operatorname{Pr}\left[X^{n}=x^{n}\right] \operatorname{Pr}\left[\bar{X}^{n}=\bar{x}^{n}\right],
\end{aligned}
$$

giving

$$
\begin{aligned}
& \operatorname{Pr}\left[\bar{X}^{n}=\bar{x}^{n} \mid X^{n}, Y^{n}\right] \\
& \leq \frac{\alpha \operatorname{Pr}\left[X^{n}=x^{n}\right] \operatorname{Pr}\left[\bar{X}^{n}=\bar{x}^{n}\right]}{\operatorname{Pr}\left[X^{n}=x^{n}\right]} \\
& =\alpha \operatorname{Pr}\left[X^{n}=x^{n}\right] \\
& =\alpha P_{X^{n}}\left(\bar{x}^{n}\right) \frac{P_{Y^{n} \mid X^{n}}\left(Y^{n} \mid \bar{x}^{n}\right)}{P_{Y^{n}}\left(Y^{n}\right)} \frac{P_{Y^{n}}\left(Y^{n}\right)}{P_{Y^{n} \mid X^{n}}\left(Y^{n} \mid \bar{x}^{n}\right)} \\
& =\alpha \operatorname{Pr}\left[X^{n}=\bar{x}^{n} \mid Y^{n}\right] \exp \left\{-i\left(\bar{x}^{n} ; Y^{n}\right)\right\} .
\end{aligned}
$$

Next, we follow the approach from the proof of Theorem 11 to show

$$
\epsilon^{\prime} \leq \mathbb{E}\left[\min \left\{1, \alpha M \frac{A\left(P_{X}\right)}{\sqrt{n}} \exp \left(-I_{n}\right)\right\}\right] .
$$

To bound $I_{n}$ using the Berry-Esséen Theorem (Lemma 12), we first need to check whether $I_{n}$ is a sum of independent random variables under the $\operatorname{LDPC}(\lambda, \rho, \delta ; n)$ ensemble. That is, we need to show

$$
\log \frac{P_{Y^{n} \mid X^{n}}\left(y^{n} \mid x^{n}\right)}{P_{Y^{n}}\left(y^{n}\right)}=\sum_{j=1}^{n} \log \frac{P_{Y \mid X}\left(y_{j} \mid x_{j}\right)}{P_{Y}\left(y_{j}\right)} .
$$

The given equality holds due to the uniform distribution of coset vector $\boldsymbol{v}$. Formally,

$$
\begin{aligned}
& \operatorname{Pr}\left[\delta\left(\left(\boldsymbol{c}_{1}+\boldsymbol{v}\right)[j]\right)=X_{j} \mid \delta\left(\left(\boldsymbol{c}_{1}+\boldsymbol{v}\right)[1: j-1]\right)=X^{j-1}\right] \\
= & \operatorname{Pr}\left[\delta\left(\left(\boldsymbol{c}_{1}+\boldsymbol{v}\right)[j]\right)=X_{j}\right], \forall j \in\{2, \ldots, n\} .
\end{aligned}
$$

Setting

$\log M=n I\left(P_{X}\right)+\frac{1}{2} \log n-\log A\left(P_{X}\right)-\frac{\log \alpha}{n}$ 


$$
-\sqrt{n V\left(P_{X}\right)} Q^{-1}\left(\epsilon-\frac{B\left(P_{X}\right)+A\left(P_{X}\right)}{\sqrt{n}}\right)
$$

and following the derivation from 927 to $(102)$, we can show 202 is bounded by $\epsilon$.

Therefore, setting $P_{X}$ to the capacity achieving distribution, and using (105) and 106) to bound $Q^{-1}\left(\epsilon-\frac{B\left(P_{X}\right)+A\left(P_{X}\right)}{\sqrt{n}}\right)$ yields the desired achievability bound.

Remark 14: Theorem 15 provides an achievability bound for the LDPC code ensemble. The result is optimal in its first- and second-order terms. The third-order term exceeds the optimal third-order term for i.i.d. codeword design in (62), providing an upper bound on the effect of LDPC codeword dependence. By this result, the penalty incurred for using the LDPC code ensemble is at most $\frac{\log \alpha}{n}$, which we show to be $O\left(\frac{\log n}{n}\right)$ if $\rho=\kappa n$ and $\kappa$ approaches zero no more quickly than $\Theta\left(\frac{\log n}{n}\right)$, provided and we first expurgate codes with low minimal distance as shown in Appendix $\mathrm{D}$

\section{B. RCU Bound for LDPC Code on the DM-2-MAC}

Just as Theorem 15 extends the proof of Theorem 11 from i.i.d. code design to LDPC code design in the PPC, Theorem 16, below, extends Theorem 14 from i.i.d. code design to LDPC code design in the MAC.

Theorem 16: (LDPC code finite-blocklength bound, and second-order best-prior achievability on the DM-2-MAC). Consider a DM-2-MAC $\left(\mathcal{X}_{1} \times \mathcal{X}_{2}, P_{Y \mid X_{1}, X_{2}}, \mathcal{Y}\right)$. Assume transmitter $i$ employs the $\operatorname{LDPC}\left(\lambda_{i}, \rho_{i}, \delta_{i} ; n\right)$ ensemble with coset vector $\boldsymbol{v}_{i}$, and quantizer $\delta_{i}(\cdot)$ chosen to approximate $P_{X_{i}}$ for $i \in\{1,2\}$. Then there exist LDPC parameters $\left(\lambda_{1}, \rho_{1}\right)$ and $\left(\lambda_{2}, \rho_{2}\right)$ for which the $\operatorname{LDPC}\left(\lambda_{1}, \rho_{1}, \delta_{1} ; n\right) \times$ $\operatorname{LDPC}\left(\lambda_{2}, \rho_{2}, \delta_{2} ; n\right)$ ensemble contains at least one MAC code with average error bounded by $\epsilon$ such that for any blocklength $n$

$$
\epsilon \leq \mathbb{E}\left[\min \left\{1, \alpha_{1} E_{1}+\alpha_{2} E_{2}+\alpha_{1} \alpha_{2} E_{12}\right\}\right],
$$

and for large enough $n$

$$
\overline{\boldsymbol{R}} \in \overline{\boldsymbol{I}}-\frac{Q_{\text {inv }}(\mathrm{V}, \epsilon)}{\sqrt{n}}+\frac{\log n}{2 n} \mathbf{1}-\frac{\log \overline{\boldsymbol{\alpha}}}{n} \mathbf{1}-O\left(\frac{1}{n}\right) \mathbf{1},
$$

provided the moment assumptions (132)-(134) are satisfied. The definitions of $\overline{\boldsymbol{I}}$ and $\mathrm{V}$ are the same as those in Theorem 14. $E_{1}, E_{2}$ and $E_{12}$ are defined in (138)-(140), and $\alpha_{1}$ and $\alpha_{2}$ are the same as those in Theorem 4 . The remaining terms are defined as

$$
\begin{aligned}
& \overline{\boldsymbol{R}} \triangleq\left[\begin{array}{c}
R_{1} \\
R_{2} \\
R_{1}+R_{2}
\end{array}\right]=\left[\begin{array}{c}
1-\frac{\lambda_{1}}{\rho_{1}} \\
1-\frac{\lambda_{2}}{\rho_{2}} \\
1-\frac{\lambda_{1}}{\rho_{1}}+1-\frac{\lambda_{2}}{\rho_{2}}
\end{array}\right], \\
& \overline{\boldsymbol{\alpha}}=\left[\begin{array}{c}
\alpha_{1} \\
\alpha_{2} \\
\alpha_{1} \alpha_{2}
\end{array}\right] .
\end{aligned}
$$

Remark 15: Since the quantizers $\delta_{i}(\cdot), i \in\{1,2\}$ restrict achievable input distributions $P_{X_{i}}, i \in\{1,2\}$ to be integer multiples of $\frac{1}{q}$, rate pairs $\left(R_{1}, R_{2}\right)$ that require irrational input distributions or rational input distributions with non-integer multiples of $\frac{1}{q}$ may require large alphabet size $q$ to closely approximate the desired input distributions.

Proof of Theorem 16. Notice that the $\operatorname{LDPC}\left(\lambda_{i}, \rho_{i}, \delta_{i} ; n\right)$ ensemble meets the codeword distribution constraint of Theorem 13 .

Setting $X_{1}=X_{1}^{n}, \bar{X}_{1}=\bar{X}_{1}^{n}, X_{2}=X_{2}^{n}, \bar{X}_{2}=$ $\bar{X}_{2}^{n}, Y=Y^{n}$ in Theorem 13, we note that there exists at least one code in the joint ensemble $\operatorname{LDPC}\left(\lambda_{1}, \rho_{1}, \delta_{1} ; n\right) \times$ $\operatorname{LDPC}\left(\lambda_{2}, \rho_{2}, \delta_{2} ; n\right)$ such that the average error probability $\epsilon^{\prime}$ satisfies

$$
\epsilon^{\prime} \leq \mathbb{E}\left[\min \left\{1, V_{1}+V_{2}+V_{12}\right\}\right]
$$

where

$$
\begin{aligned}
V_{1}= & \left(M_{1}-1\right) \\
& \quad \operatorname{Pr}\left[i\left(\bar{X}_{1}^{n} ; Y^{n} \mid X_{2}^{n}\right) \geq i\left(X_{1}^{n} ; Y^{n} \mid X_{2}^{n}\right) \mid X_{1}^{n}, X_{2}^{n}, Y^{n}\right], \\
V_{2}= & \left(M_{2}-1\right) \\
& \quad \operatorname{Pr}\left[i\left(\bar{X}_{2}^{n} ; Y^{n} \mid X_{1}^{n}\right) \geq i\left(X_{2}^{n} ; Y^{n} \mid X_{1}^{n}\right) \mid X_{1}^{n}, X_{2}^{n}, Y^{n}\right], \\
V_{12}= & \left(M_{1}-1\right)\left(M_{2}-1\right) \\
& \left.\quad \operatorname{Pr}\left[i\left(\bar{X}_{1}^{n}, \bar{X}_{2}^{n} ; Y^{n}\right) \geq i\left(X_{1}^{n}, X_{2}^{n} ; Y\right)^{n} \mid X_{1}^{n}, X_{2}^{n}, Y^{n}\right]\right\} .
\end{aligned}
$$

Since the codebooks for transmitter 1 and transmitter 2 are independently designed, but the codewords in each are dependent under LDPC design

$$
\begin{aligned}
& P_{X_{1}^{n} X_{2}^{n} \bar{X}_{1}^{n} \bar{X}_{2}^{n} Y^{n}}\left(x_{1}^{n}, x_{2}^{n}, \bar{x}_{1}^{n}, \bar{x}_{2}^{n}, y^{n}\right) \\
& =P_{X_{1}^{n} \bar{X}_{1}^{n}}\left(x_{1}^{n}, \bar{x}_{1}^{n}\right) P_{X_{2}^{n} \bar{X}_{2}^{n}}\left(x_{2}^{n}, \bar{x}_{2}^{n}\right) P_{Y^{n} \mid X_{1}^{n} X_{2}^{n}}\left(y^{n} \mid x_{1}^{n}, x_{2}^{n}\right) .
\end{aligned}
$$

From Appendix A we know that for each $i \in\{1,2\}$

$$
\operatorname{Pr}\left[\boldsymbol{C}_{i, 1}+\boldsymbol{v}_{i}=\boldsymbol{a}, \boldsymbol{C}_{i, 2}+\boldsymbol{v}_{i}=\boldsymbol{a}^{\prime}\right] \leq q^{-n} \alpha_{i} q^{-n},
$$

where $\boldsymbol{C}_{i, 1}$ and $\boldsymbol{C}_{i, 2}$ are the codewords for messages 1 and 2 from a random code in the $\operatorname{LDPC}\left(\lambda_{i}, \rho_{i}, \delta_{i} ; n\right)$ ensemble for transmitter $i$, and $\boldsymbol{v}_{i}$ is the coset vector for transmitter $i$.

For each of the LDPC code ensembles, we note that for $i \in\{1,2\}, \bar{X}_{i}^{n} \rightarrow X_{i}^{n} \rightarrow\left(X_{3-i}^{n}, Y^{n}\right)$ forms a Markov chain, as the dependence of $Y^{n}$ or $X_{3-i}^{n}$ on $\bar{X}_{i}^{n}$ is through $X_{i}^{n}$. A given $Y^{n}$ affects the conditional distribution on $X_{i}^{n}$ through the structure of the channel, and thus affects the conditional distribution of $\bar{X}_{i}^{n}$ through the dependence between $X_{i}^{n}$ and $\bar{X}_{i}^{n}$. By the assumption of independent coset vectors $\boldsymbol{v}_{1}$ and $\boldsymbol{v}_{2}, X_{3-i}^{n}$ is independent of $\bar{X}_{i}^{n}$.

Therefore,

$$
\begin{aligned}
\operatorname{Pr}\left[\bar{X}_{1}^{n}=\bar{x}_{1}^{n} \mid X_{1}^{n}, X_{2}^{n}, Y^{n}\right] & =\operatorname{Pr}\left[\bar{X}_{1}^{n}=\bar{x}_{1}^{n} \mid X_{1}^{n}\right] \\
& =\frac{\operatorname{Pr}\left[\bar{X}^{n}=\bar{x}^{n}, X^{n}=x^{n}\right]}{\operatorname{Pr}\left[X^{n}=x^{n}\right]} .
\end{aligned}
$$

By an argument similar to (192)-(196), we have

$$
\begin{aligned}
& \operatorname{Pr}\left[\bar{X}_{1}^{n}=\bar{x}_{1}^{n} \mid X_{1}^{n}, X_{2}^{n}, Y^{n}\right] \\
& \leq \alpha_{1} \operatorname{Pr}\left[\bar{X}_{1}^{n}=\bar{x}_{1}^{n}\right] \\
& =\alpha_{1} \operatorname{Pr}\left[X_{1}^{n}=\bar{x}_{1}^{n} \mid Y^{n}, X_{2}^{n}\right] \exp \left\{-i\left(\bar{x}_{1}^{n} ; Y^{n} \mid X_{2}^{n}\right)\right\} .
\end{aligned}
$$


Summing over all $\bar{x}_{1}^{n}=i\left(\bar{x}_{1}^{n} ; Y^{n} \mid X_{2}^{n}\right)$ such that $\bar{I}_{1 n} \geq \zeta$ gives

$$
\begin{aligned}
& \operatorname{Pr}\left[\bar{I}_{1 n} \geq \zeta \mid Y^{n}, X_{2}^{n}\right] \\
& \quad=\alpha_{1} \mathbb{E}\left[\exp \left\{-\bar{I}_{1 n}\right\} \mathbb{1}\left\{\bar{I}_{1 n} \geq \zeta\right\} \mid Y^{n}, X_{2}^{n}\right] \\
& \quad \leq \quad \alpha_{1} \frac{F_{1}}{\sqrt{n}} \exp (-\zeta),
\end{aligned}
$$

where (220) follows from Lemma 1, and $F_{1}$ is defined in (135).

Therefore,

$$
V_{1} \leq \alpha_{1} \frac{F_{1}}{\sqrt{n}} \exp \left(-i\left(X_{1}^{n} ; Y^{n} \mid X_{2}^{n}\right)\right)
$$

Switching the role of transmitter 1 and transmitter 2 yields

$$
\begin{aligned}
& \operatorname{Pr}\left[\bar{X}_{2}^{n}=\bar{x}_{2}^{n} \mid X_{1}^{n}, X_{2}^{n}, Y^{n}\right] \\
& \leq \alpha_{2} \operatorname{Pr}\left[X_{2}^{n}=\bar{x}_{2}^{n} \mid Y^{n}, X_{1}^{n}\right] \exp \left\{-i\left(\bar{x}_{2}^{n} ; Y^{n} \mid X_{1}^{n}\right)\right\},
\end{aligned}
$$

and therefore

$$
V_{2} \leq \alpha_{1} \frac{F_{2}}{\sqrt{n}} \exp \left(-i\left(X_{2}^{n} ; Y^{n} \mid X_{1}^{n}\right)\right) .
$$

Finally, for $\operatorname{Pr}\left[\bar{X}_{1}^{n}=\bar{x}_{1}^{n}, \bar{X}_{2}^{n}=\bar{x}_{2}^{n} \mid X_{1}^{n}, X_{2}^{n}, Y^{n}\right]$, we have

$$
\begin{aligned}
& \operatorname{Pr}\left[\bar{X}_{1}^{n}=\bar{x}_{1}^{n}, \bar{X}_{2}^{n}=\bar{x}_{2}^{n} \mid X_{1}^{n}, X_{2}^{n}, Y^{n}\right] \\
& =\operatorname{Pr}\left[\bar{X}_{2}^{n}=\bar{x}_{2}^{n} \mid X_{1}^{n}, X_{2}^{n}, Y^{n}\right] \\
& \quad \operatorname{Pr}\left[\bar{X}_{1}^{n}=\bar{x}_{1}^{n}, \mid X_{1}^{n}, X_{2}^{n}, Y^{n}, \bar{X}_{2}^{n}\right] \\
& =\operatorname{Pr}\left[\bar{X}_{2}^{n}=\bar{x}_{2}^{n} \mid X_{2}^{n}\right] \operatorname{Pr}\left[\bar{X}_{1}^{n}=\bar{x}_{1}^{n}, \mid X_{1}^{n}\right] \\
& \leq \alpha_{1} \alpha_{2} \operatorname{Pr}\left[X_{1}^{n}=\bar{x}_{1}^{n}, X_{2}^{n}=\bar{x}_{2}^{n} \mid Y^{n}\right] \exp \left\{-i\left(\bar{x}_{1}^{n}, \bar{x}_{2}^{n} ; Y^{n}\right)\right\},
\end{aligned}
$$

and

$$
V_{12} \leq \alpha_{1} \alpha_{2} \frac{F_{12}}{\sqrt{n}} \exp \left(-i\left(X_{1}^{n}, X_{2}^{n} ; Y^{n}\right)\right) .
$$

With the definitions of $E_{1}, E_{2}, E_{12}$ from (138)-(140), combining the above three results on $V_{1}, V_{2}$, and $V_{12}$ gives the finite-blocklength error bound.

The rest of the proof follows from the proof of Theorem 14 by invoking Berry-Esséen Theorem (Lemma 12) to bound each of the three terms in (221), (224), and (229), which gives the following achievability result for large enough $n$

$$
\overline{\boldsymbol{R}} \in \overline{\boldsymbol{I}}-\frac{Q_{\mathrm{inv}}(\mathrm{V}, \epsilon)}{\sqrt{n}}+\frac{\log n}{2 n} \mathbf{1}-\frac{\log \overline{\boldsymbol{\alpha}}}{n} \mathbf{1}-O\left(\frac{1}{n}\right) \mathbf{1} .
$$

Remark 16: Theorem 16 provides an achievability bound for the random LPDC ensemble that achieves the same secondorder term as the best known bound for i.i.d. MAC codes. The penalty for the codeword dependence that result from using the LDPC code ensemble is the $\frac{\log \bar{\alpha}}{n}$ term, which is $O\left(\frac{\log n}{n}\right) \mathbf{1}$ if $\rho_{1}=\kappa_{1} n, \rho_{2}=\kappa_{2} n$ and $\kappa_{1}, \kappa_{2}$ approach zero no more quickly than $\Theta\left(\frac{\log n}{n}\right)$, provided that we first expurgate codes with small minimal distance.

Remark 17: The proof of Theorem 16 uses an independent code ensemble for each transmitter and independent coset vectors. For many practical scenarios, it is useful to allow different transmitters to use the same LDPC code for simplicity. If the same code ensemble $\operatorname{LDPC}(\lambda, \rho ; n)$ (before applying the coset vector and quantization) is used for both transmitters, then $\alpha_{1}=\alpha_{2}$. In addition, if the transmitters use the same coset vector, then both $X_{1}^{n}$ and $X_{2}^{n}$ have an impact on the distribution of $\bar{X}_{1}^{n}$ (similar for $\bar{X}_{2}^{n}$ ), as knowing both $X_{1}^{n}, X_{2}^{n}$ (assuming $X_{1}^{n} \neq X_{2}^{n}$ ) reveals two different codewords in the codebook. In this case, the penalty term $\log \overline{\boldsymbol{\alpha}}$ becomes

$$
\log \overline{\boldsymbol{\alpha}}=\left[\begin{array}{c}
2 \log \alpha_{1} \\
2 \log \alpha_{2} \\
2 \log \alpha_{1}+2 \log \alpha_{2}
\end{array}\right]=\left[\begin{array}{c}
2 \log \alpha_{1} \\
2 \log \alpha_{1} \\
4 \log \alpha_{1}
\end{array}\right] .
$$

Hence, different transmitters may use the same or different coset vectors depending on their sensitivity to the factor of 2 difference in the rate penalty bound.

\section{SUMmary AND CONCLUSIONS}

This paper studies the performance of quantized coset LDPC codes over the DM-PPC and the DM-MAC using finiteblocklength and error-exponent analyses.

For the error-exponent analysis, we extend the result of [4] from the DM-PPC to symmetrical rates in the symmetric DM- $K$-MAC and arbitrary rates in the general DM-2-MAC using Gallager's error exponent. A non-asymptotic expansion of Gallager's error exponent is provided using [6. Exercise 5.23].

For the dispersion-style approach, we derive finiteblocklength error bounds and asymptotic third-order achievability results for the DM-PPC and the DM-2-MAC for standard i.i.d. codes; the achievability result is optimal up to the third order for the DM-PPC (Theorem 111), and is the tightest bound available to date for the DM-2-MAC (Theorem 14). Application of two generalized RCU bounds (Theorem 10 for the DM-PPC and Theorem 13 for the DM-2MAC) shows that quantized coset LDPC codes achieve firstand second-order performance that is optimal for the DMPPC (Theorem 15) and identical to the best-prior results for the DM-MAC (Theorem 16), provided that we first expurgate LDPC codes with small minimum distance, and the sparsity of LDPC codes $\left(\kappa=\frac{\rho}{n}\right)$ decays no more quickly than $\Theta\left(\frac{\log n}{n}\right)$.

A comparison of both approaches (Section III-C) demonstrates that the error-exponent analysis achieves a sub-optimal second-order coefficient in blocklength $n$ but a superior bound when target error probability $\epsilon$ is small.

\section{APPENDIX A \\ Proof of Theorem 1}

Recall that by the given code construction, all transmitters employ the same codebook, but each is offset by an independent random coset vector. Recall further that the codebook is restricted to include precisely $M=q^{n R}$ codewords, where $R=1-\frac{\lambda}{\rho}$ is the design rate. Here $\boldsymbol{c}=\left\{\boldsymbol{c}_{1}, \ldots, \boldsymbol{c}_{M}\right\}$ describes the single-transmitter codebook and $\boldsymbol{d}=\left\{\boldsymbol{d}_{\boldsymbol{m}}: \boldsymbol{m} \in[M]^{K}\right\}$ describes the corresponding MAC codebook, where for any $\boldsymbol{m}=(m(1), \ldots, m(K)), \boldsymbol{d}_{\boldsymbol{m}}=\left(\boldsymbol{c}_{m(1)}, \ldots, \boldsymbol{c}_{m(K)}\right)$. Given a coset matrix $\boldsymbol{v}$ and quantizer $\delta$, the resulting set of channel inputs is $\left\{\delta\left(\boldsymbol{d}_{\boldsymbol{m}}+\boldsymbol{v}\right): \boldsymbol{m} \in[M]^{K}\right\}$. 
The expected value under our random code construction of the average error probability is

$$
\begin{aligned}
E\left[P_{e}^{(n)}\right] & =\sum_{\boldsymbol{m}} \sum_{\boldsymbol{d}} \sum_{\boldsymbol{v}} P_{\boldsymbol{M}}(\boldsymbol{m}) P_{\boldsymbol{D}}(\boldsymbol{d}) P_{\boldsymbol{V}}(\boldsymbol{v}) P_{e \mid \boldsymbol{m}, \boldsymbol{d}, \boldsymbol{v}}^{(n)} \\
& =E_{\boldsymbol{M} \boldsymbol{D} \boldsymbol{V}}\left[P_{e \mid \boldsymbol{M}, \boldsymbol{D}, \boldsymbol{V}}^{(n)}\right]
\end{aligned}
$$

where $P_{e \mid \boldsymbol{m}, \boldsymbol{d}, \boldsymbol{v}}^{(n)}$ is the conditional error probability under fixed values of the message vector $\boldsymbol{m}$, codebook $\boldsymbol{d}$, and coset matrix $\boldsymbol{v}, P_{\boldsymbol{M}}(\boldsymbol{m}), P_{\boldsymbol{D}}(\boldsymbol{d})$, and $P_{\boldsymbol{V}}(\boldsymbol{v})$ capture the (independent, uniform) distributions on the vectors of possible messages, set of possible codebooks, and cosets, respectively, and $E_{M D V}[\cdot]$ is the resulting expectation.

We begin by bounding the conditional error probability $P_{e \mid \boldsymbol{m}, \boldsymbol{d}, \boldsymbol{v}}^{(N)}$. Let

$$
\begin{aligned}
\mathcal{Y}_{\boldsymbol{m}, \boldsymbol{d}, \boldsymbol{v}}= & \left\{\boldsymbol{y}: \exists \boldsymbol{m}^{\prime} \in[M]^{K} \backslash\{\boldsymbol{m}\}\right. \text { s.t. } \\
& \left.\operatorname{Pr}\left[\boldsymbol{y} \mid \delta\left(\boldsymbol{d}_{\boldsymbol{m}^{\prime}}+\boldsymbol{v}\right)\right] \geq \operatorname{Pr}\left[\boldsymbol{y} \mid \delta\left(\boldsymbol{d}_{\boldsymbol{m}}+\boldsymbol{v}\right)\right]\right\},
\end{aligned}
$$

denote the set of channel outputs for which message vector $\boldsymbol{m}$ is not the unique most likely explanation. Then

$$
P_{e \mid \boldsymbol{m}, \boldsymbol{d}, \boldsymbol{v}}^{(n)} \leq \operatorname{Pr}\left[\mathcal{Y}_{\boldsymbol{m}, \boldsymbol{d}, \boldsymbol{v}} \mid \boldsymbol{m}, \boldsymbol{d}, \boldsymbol{v}\right],
$$

which is an inequality rather than an equality since an error is not guaranteed when $\operatorname{Pr}\left[\boldsymbol{y} \mid \delta\left(\boldsymbol{d}_{\boldsymbol{m}^{\prime}}+\boldsymbol{v}\right)\right]=\operatorname{Pr}\left[\boldsymbol{y} \mid \delta\left(\boldsymbol{d}_{\boldsymbol{m}}+\boldsymbol{v}\right)\right]$. For any set $\mathrm{T} \subseteq \mathcal{T}_{\mathcal{Q}}^{n}$, define $\mathcal{Y}_{\boldsymbol{m}, \boldsymbol{d}, \boldsymbol{v}}^{\mathrm{T}}$ as

$$
\begin{array}{r}
\mathcal{Y}_{\boldsymbol{m}, \boldsymbol{d}, \boldsymbol{v}}^{\mathrm{T}}=\left\{\boldsymbol{y}: \exists \boldsymbol{m}^{\prime} \in[M]^{K} \backslash\{\boldsymbol{m}\} \text { s.t. } \mathcal{T}_{\mathcal{Q}}^{n}\left(\Delta_{\boldsymbol{m}, \boldsymbol{m}^{\prime}}^{\boldsymbol{d}}\right) \in \mathrm{T}\right. \\
\text { and } \left.\operatorname{Pr}\left[\boldsymbol{y} \mid \delta\left(\boldsymbol{d}_{\boldsymbol{m}^{\prime}}+\boldsymbol{v}\right)\right] \geq \operatorname{Pr}\left[\boldsymbol{y} \mid \delta\left(\boldsymbol{d}_{\boldsymbol{m}}+\boldsymbol{v}\right)\right]\right\},
\end{array}
$$

where

$$
\Delta_{m^{\prime}, m}^{d}=d_{m}-d_{m^{\prime}} .
$$

Recall that $\mathrm{T}^{c}=\mathcal{T}_{\mathcal{Q}}^{n} \backslash \mathrm{T} \backslash\left\{\mathcal{T}_{\mathcal{Q}}^{n}(\mathbf{0})\right\}$, where $\mathbf{0}$ is the all-zeros codematrix. Then

$$
\mathcal{Y}_{\boldsymbol{m}, \boldsymbol{d}, \boldsymbol{v}}=\mathcal{Y}_{\boldsymbol{m}, \boldsymbol{d}, \boldsymbol{v}}^{\mathrm{T}} \cup \mathcal{Y}_{\boldsymbol{m}, \boldsymbol{d}, \boldsymbol{v}}^{\mathrm{T}^{c}} \cup \mathcal{Y}_{\boldsymbol{m}, \boldsymbol{d}, \boldsymbol{v}}^{\left\{\mathcal{T}_{Q}^{n}(\mathbf{0})\right\}},
$$

and we have

$$
\begin{aligned}
P_{e \mid \boldsymbol{m}, \boldsymbol{d}, \boldsymbol{v}}^{(n)} \leq & \operatorname{Pr}\left[\mathcal{Y}_{\boldsymbol{m}, \boldsymbol{d}, \boldsymbol{v}}^{\mathrm{T}} \mid \boldsymbol{m}, \boldsymbol{d}, \boldsymbol{v}\right]+\operatorname{Pr}\left[\mathcal{Y}_{\boldsymbol{m}, \boldsymbol{d}, \boldsymbol{v}}^{\mathrm{T}^{c}} \mid \boldsymbol{m}, \boldsymbol{d}, \boldsymbol{v}\right] \\
& +\operatorname{Pr}\left[\mathcal{Y}_{\boldsymbol{m}, \boldsymbol{d}, \boldsymbol{v}}^{\left\{\mathcal{T}_{\mathcal{Q}}^{n}(\mathbf{0})\right\}} \mid \boldsymbol{m}, \boldsymbol{d}, \boldsymbol{v}\right] .
\end{aligned}
$$

Since all codewords in the single-transmitter codebook are distinct by definition $\left(\boldsymbol{c}_{m} \neq \boldsymbol{c}_{m^{\prime}}\right.$ for all $\left.m^{\prime} \in[M] \backslash\{m\}\right)$, all codematrices are also distinct $\left(\boldsymbol{d}_{\boldsymbol{m}} \neq \boldsymbol{d}_{\boldsymbol{m}^{\prime}}\right.$ for all $\boldsymbol{d}$ and all $\left.\boldsymbol{m}^{\prime} \in[M]^{K} \backslash\{\boldsymbol{m}\}\right)$, set $\mathcal{Y}_{\boldsymbol{m}, \boldsymbol{d}, \boldsymbol{v}}^{\left\{\mathcal{T}_{\mathcal{Q}}^{n}(\mathbf{0})\right.}$ is always empty, and we can bound $P_{e \mid \boldsymbol{m}, \boldsymbol{d}, \boldsymbol{v}}$ by bounding the remaining two terms.

Let $\boldsymbol{t}_{\boldsymbol{m}, \boldsymbol{m}^{\prime}}=\mathcal{T}_{\mathcal{Q}}^{n}\left(\Delta_{\boldsymbol{m}, \boldsymbol{m}^{\prime}}^{\boldsymbol{d}}\right)$. Then, for the first term,

$$
\begin{aligned}
& \operatorname{Pr}\left[\mathcal{Y}_{\boldsymbol{m}, \boldsymbol{d}, \boldsymbol{v}}^{\mathrm{T}} \mid \boldsymbol{m}, \boldsymbol{d}, \boldsymbol{v}\right] \\
& =\sum_{\boldsymbol{y} \in \mathcal{Y}_{\boldsymbol{m}, \boldsymbol{d}, \boldsymbol{v}}^{\mathrm{T}}} P_{\boldsymbol{Y} \mid \boldsymbol{X}}\left(\boldsymbol{y} \mid \delta\left(\boldsymbol{d}_{\boldsymbol{m}}+\boldsymbol{v}\right)\right) \\
& \leq \sum_{\boldsymbol{y} \in \mathcal{Y}_{\boldsymbol{m}, \boldsymbol{d}, \boldsymbol{v}}^{\mathrm{T}}}\left[P_{\boldsymbol{Y} \mid \boldsymbol{X}}\left(\boldsymbol{y} \mid \delta\left(\boldsymbol{d}_{\boldsymbol{m}}+\boldsymbol{v}\right)\right)\right. \\
& \left.\sum_{\boldsymbol{m}^{\prime}: t_{\boldsymbol{m}, \boldsymbol{m}^{\prime}} \in \mathrm{T}} \sqrt{\frac{P_{\boldsymbol{Y} \mid \boldsymbol{X}}\left(\boldsymbol{y} \mid \delta\left(\boldsymbol{d}_{\boldsymbol{m}^{\prime}}+\boldsymbol{v}\right)\right)}{P_{\boldsymbol{Y} \mid \boldsymbol{X}}\left(\boldsymbol{y} \mid \delta\left(\boldsymbol{d}_{\boldsymbol{m}}+\boldsymbol{v}\right)\right)}}\right]
\end{aligned}
$$

$$
\begin{aligned}
& \leq \sum_{\boldsymbol{y}} \sum_{\boldsymbol{m}^{\prime}: \boldsymbol{t}_{\boldsymbol{m}, \boldsymbol{m}^{\prime}} \in \mathrm{T}} \\
& \sqrt{P_{\boldsymbol{Y} \mid \boldsymbol{X}}\left(\boldsymbol{y} \mid \delta\left(\boldsymbol{d}_{\boldsymbol{m}^{\prime}}+\boldsymbol{v}\right)\right) P_{\boldsymbol{Y} \mid \boldsymbol{X}}\left(\boldsymbol{y} \mid \delta\left(\boldsymbol{d}_{\boldsymbol{m}}+\boldsymbol{v}\right)\right)} \\
& =\sum_{\boldsymbol{m}^{\prime}: \boldsymbol{t}_{\boldsymbol{m}, \boldsymbol{m}^{\prime}} \in \mathrm{T}} \sum_{\boldsymbol{y}} \prod_{i=1}^{n} \\
& \sqrt{P_{Y \mid X}\left(y_{i} \mid \delta\left(\left(\boldsymbol{d}_{\boldsymbol{m}^{\prime}}+\boldsymbol{v}\right)[i, *]\right)\right) P_{Y \mid X}\left(y_{i} \mid \delta\left(\left(\boldsymbol{d}_{\boldsymbol{m}}+\boldsymbol{v}\right)[i, *]\right)\right)} \\
& =\sum_{\boldsymbol{m}^{\prime}: \boldsymbol{t}_{\boldsymbol{m}, \boldsymbol{m}^{\prime}} \in \mathrm{T}} \prod_{i=1}^{n} \sum_{y} \\
& \sqrt{P_{Y \mid X}\left(y \mid \delta\left(\left(\boldsymbol{d}_{\boldsymbol{m}^{\prime}}+\boldsymbol{v}\right)[i, *]\right)\right) P_{Y \mid X}\left(y \mid \delta\left(\left(\boldsymbol{d}_{\boldsymbol{m}}+\boldsymbol{v}\right)[i, *]\right)\right)} .
\end{aligned}
$$

Taking the expectation over random cosets gives

$$
\begin{aligned}
& E_{\boldsymbol{V}}\left[\operatorname{Pr}\left[\mathcal{Y}_{\boldsymbol{m}, \boldsymbol{d}, \boldsymbol{V}}^{\mathrm{T}} \mid \boldsymbol{m}, \boldsymbol{d}, \boldsymbol{V}\right]\right] \\
& \leq E_{\boldsymbol{V}}\left[\sum_{\boldsymbol{m}^{\prime}: \boldsymbol{t}_{\boldsymbol{m}, \boldsymbol{m}^{\prime}} \in \mathrm{T}} \prod_{i=1}^{n} \sum_{y}\right. \\
& \left.\sqrt{P_{Y \mid X}\left(y \mid \delta\left(\left(\boldsymbol{d}_{\boldsymbol{m}^{\prime}}+\boldsymbol{V}\right)[i, *]\right)\right) P_{Y \mid X}\left(y \mid \delta\left(\left(\boldsymbol{d}_{\boldsymbol{m}}+\boldsymbol{V}\right)[i, *]\right)\right)}\right] \\
& =\sum_{\boldsymbol{m}^{\prime}: \boldsymbol{t}_{\boldsymbol{m}, \boldsymbol{m}^{\prime}} \in \mathrm{T}} \prod_{i=1}^{n} E_{\boldsymbol{V}[i, *]}\left[\sum_{y}\right. \\
& \left.\sqrt{P_{Y \mid X}\left(y \mid \delta\left(\left(\boldsymbol{d}_{\boldsymbol{m}^{\prime}}+\boldsymbol{V}\right)[i, *]\right)\right) P_{Y \mid X}\left(y \mid \delta\left(\left(\boldsymbol{d}_{\boldsymbol{m}}+\boldsymbol{V}\right)[i, *]\right)\right)}\right]
\end{aligned}
$$$$
\stackrel{(a)}{=} \sum_{\boldsymbol{m}^{\prime}: \boldsymbol{t}_{\boldsymbol{m}, \boldsymbol{m}^{\prime}} \in \mathrm{T}} \prod_{i=1}^{n}\left[\sum_{g^{\prime} \in \mathcal{Q}} \frac{1}{q^{K}} \sum_{y}\right.
$$$$
\left.\sqrt{P_{Y \mid X}\left(y \mid \delta\left(g^{\prime}+\Delta_{\boldsymbol{m}, \boldsymbol{m}^{\prime}}^{\boldsymbol{d}}[i, *]\right)\right) P_{Y \mid X}\left(y \mid \delta\left(g^{\prime}\right)\right)}\right]
$$$$
\stackrel{(b)}{=} \sum_{\boldsymbol{m}^{\prime}: \boldsymbol{t}_{\boldsymbol{m}, \boldsymbol{m}^{\prime}} \in \mathrm{T}} \prod_{i=1}^{n} \mathcal{D}\left(\Delta_{\boldsymbol{m}, \boldsymbol{m}^{\prime}}^{\boldsymbol{d}}[i, *]\right)
$$$$
\stackrel{(c)}{=} \sum_{m^{\prime}: t_{m, m^{\prime}} \in \mathrm{T}} \mathcal{D}^{t_{m, m^{\prime}}}
$$$$
\stackrel{(d)}{\leq} \sum_{m^{*}: \mathcal{T}_{\mathcal{Q}}^{n}\left(\boldsymbol{d}_{m^{*}}\right) \in \mathrm{T}} \mathcal{D}^{\mathcal{T}_{\mathcal{Q}}^{n}\left(\boldsymbol{d}_{m^{*}}\right)}
$$$$
\stackrel{(e)}{=} \sum_{\boldsymbol{t} \in \mathrm{T}} S_{\boldsymbol{d}}^{n}(\boldsymbol{t}) \mathcal{D}^{t}
$$

Here $(a)$ follows since each row of $\boldsymbol{V}$ is uniformly distributed over $\mathcal{Q}$, which implies that each row of $\boldsymbol{d}_{\boldsymbol{m}}+\boldsymbol{V}$ is uniformly distributed over $\mathcal{Q} ;(b)$ and $(c)$ apply definitions $(18)$ and $(19)$; $(d)$ uses the fact that the difference between two codewords is a codeword in any liner code, and therefore the given upper bound applies after our random selection of codewords; and (e) applies definition (16). Finally, taking the expectation with respect to the random choice of the codebook and message gives

$$
E\left[\operatorname{Pr}\left[\mathcal{Y}_{\boldsymbol{M}, \boldsymbol{D}, \boldsymbol{V}}^{\mathrm{T}} \mid \boldsymbol{M}, \boldsymbol{D}, \boldsymbol{V}\right]\right] \leq \sum_{\boldsymbol{t} \in \mathrm{T}} \overline{\boldsymbol{S}}^{n}(\boldsymbol{t}) \mathcal{D}^{\boldsymbol{t}} .
$$


For the second term, abbreviating $\boldsymbol{m} \in[M]^{K} \backslash\{\boldsymbol{m}\}$ to $\boldsymbol{m} \neq \boldsymbol{m}^{\prime}$

$$
\begin{aligned}
E & {\left[\operatorname{Pr}\left[\mathcal{Y}_{M, \boldsymbol{D}, \boldsymbol{V}}^{\mathrm{T}} \mid \boldsymbol{M}, \boldsymbol{D}, \boldsymbol{V}\right]\right] } \\
= & \sum_{\boldsymbol{m}, \boldsymbol{a}, \boldsymbol{y}} P_{\boldsymbol{M}}(\boldsymbol{m}) P_{\boldsymbol{D}_{\boldsymbol{m}}+\boldsymbol{V}}(\boldsymbol{a}) P_{\boldsymbol{Y} \mid \boldsymbol{X}}(\boldsymbol{y} \mid \delta(\boldsymbol{a})) \\
& \cdot \operatorname{Pr}\left[\exists \boldsymbol{m}^{\prime} \neq \boldsymbol{m}: \mathcal{T}_{\mathcal{Q}}^{n}\left(\boldsymbol{D}_{\boldsymbol{m}^{\prime}}-\boldsymbol{D}_{\boldsymbol{m}}\right) \in \mathrm{T}^{c},\right. \\
& P_{\boldsymbol{Y} \mid \boldsymbol{X}}\left(\boldsymbol{y} \mid \delta\left(\boldsymbol{D}_{\boldsymbol{m}^{\prime}}+\boldsymbol{V}\right)\right) \geq P_{\boldsymbol{Y} \mid \boldsymbol{X}}\left(\boldsymbol{y} \mid \delta\left(\boldsymbol{D}_{\boldsymbol{m}}+\boldsymbol{V}\right)\right) \\
& \left.\mid \boldsymbol{D}_{\boldsymbol{m}}+\boldsymbol{V}=\boldsymbol{a}\right] \\
= & \sum_{\boldsymbol{m}, \boldsymbol{a}, \boldsymbol{y}} P_{\boldsymbol{M}}(\boldsymbol{m}) P_{\boldsymbol{D}_{\boldsymbol{m}}+\boldsymbol{V}}(\boldsymbol{a}) P_{\boldsymbol{Y} \mid \boldsymbol{X}}(\boldsymbol{y} \mid \delta(\boldsymbol{a})) \\
& \cdot \operatorname{Pr}\left[\exists \boldsymbol{m}^{\prime} \neq \boldsymbol{m}: \boldsymbol{D}_{\boldsymbol{m}^{\prime}}+\boldsymbol{V}=\boldsymbol{a}^{\prime}, \mathcal{T}_{\mathcal{Q}}^{n}\left(\boldsymbol{a}^{\prime}-\boldsymbol{a}\right) \in \mathrm{T}^{c},\right. \\
& \left.P_{\boldsymbol{Y} \mid \boldsymbol{X}}\left(\boldsymbol{y} \mid \delta\left(\boldsymbol{a}^{\prime}\right)\right) \geq P_{\boldsymbol{Y} \mid \boldsymbol{X}}(\boldsymbol{y} \mid \delta(\boldsymbol{a})) \mid \boldsymbol{D}_{\boldsymbol{m}}+\boldsymbol{V}=\boldsymbol{a}\right] \\
(e) & \sum_{\boldsymbol{m}, \boldsymbol{a}, \boldsymbol{y}} P_{\boldsymbol{M}}(\boldsymbol{m}) P_{\boldsymbol{D}_{m}+\boldsymbol{V}}(\boldsymbol{a}) P_{\boldsymbol{Y} \mid \boldsymbol{X}}(\boldsymbol{y} \mid \delta(\boldsymbol{a})) \min \left\{1, \sum_{\boldsymbol{m}^{\prime} \neq \boldsymbol{m}}\right. \\
& \left.\sum_{\boldsymbol{a}_{\boldsymbol{Y} \mid \boldsymbol{X}}\left(\boldsymbol{y} \mid \delta\left(\boldsymbol{a}^{\prime}\right)\right) \geq P_{\boldsymbol{Y} \mid \boldsymbol{X}}(\boldsymbol{y} \mid \delta(\boldsymbol{a}))} \operatorname{Pr}\left[\boldsymbol{D}_{\boldsymbol{m}^{\prime}}+\boldsymbol{V}=\boldsymbol{a}^{\prime} \mid \boldsymbol{D}_{\boldsymbol{m}}+\boldsymbol{V}=\boldsymbol{a}\right]\right\}
\end{aligned}
$$$$
\stackrel{(f)}{\leq} \sum_{\boldsymbol{m}, \boldsymbol{a}, \boldsymbol{y}} P_{\boldsymbol{M}}(\boldsymbol{m}) P_{\boldsymbol{D}_{\boldsymbol{m}}+\boldsymbol{V}}(\boldsymbol{a}) P_{\boldsymbol{Y} \mid \boldsymbol{X}}(\boldsymbol{y} \mid \delta(\boldsymbol{a}))\left(\sum_{\boldsymbol{m}^{\prime} \neq \boldsymbol{m}}\right.
$$

$$
\left.\sum_{\boldsymbol{a}^{\prime}: \mathcal{T}_{\mathcal{Q}}^{n}\left(\boldsymbol{a}^{\prime}-\boldsymbol{a}\right) \in \mathrm{T}^{c}} \operatorname{Pr}\left[\boldsymbol{D}_{\boldsymbol{m}^{\prime}}+\boldsymbol{V}=\boldsymbol{a}^{\prime} \mid \boldsymbol{D}_{\boldsymbol{m}}+\boldsymbol{V}=\boldsymbol{a}\right]\right)^{\rho}
$$$$
P_{\boldsymbol{Y} \mid \boldsymbol{X}}\left(\boldsymbol{y} \mid \delta\left(\boldsymbol{a}^{\prime}\right)\right) \geq P_{\boldsymbol{Y} \mid \boldsymbol{X}}(\boldsymbol{y} \mid \delta(\boldsymbol{a}))
$$

$\stackrel{(g)}{=} \sum_{\boldsymbol{y}, \boldsymbol{a}} P_{\boldsymbol{D}_{\mathbf{1}}+\boldsymbol{V}}(\boldsymbol{a}) P_{\boldsymbol{Y} \mid \boldsymbol{X}}(\boldsymbol{y} \mid \delta(\boldsymbol{a}))\left(\sum_{\boldsymbol{m}^{\prime} \neq \mathbf{1}}\right.$

$$
\left.\sum_{\boldsymbol{a}: \mathcal{T}_{\mathcal{Q}}^{n}\left(\boldsymbol{a}^{\prime}-\boldsymbol{a}\right) \in \mathrm{T}^{c}} \operatorname{Pr}\left[\boldsymbol{D}_{\boldsymbol{m}^{\prime}}+\boldsymbol{V}=\boldsymbol{a}^{\prime} \mid \boldsymbol{D}_{\mathbf{1}}+\boldsymbol{V}=\boldsymbol{a}\right]\right)^{\rho},
$$$$
P_{\boldsymbol{Y} \mid \boldsymbol{X}}\left(\boldsymbol{y} \mid \delta\left(\boldsymbol{a}^{\prime}\right)\right) \geq P_{\boldsymbol{Y} \mid \boldsymbol{X}}(\boldsymbol{y} \mid \delta(\boldsymbol{a}))
$$

where $(e)$ follows from the union bound and the bounded nature of probabilities; $(f)$ follows by a case analysis for any $\rho \in[0,1]\left(\min \{1, a\}=1 \leq a^{\rho}\right.$ when $a \geq 1$, and $\min \{1, a\}=$ $a \leq a^{\rho}$ when $\left.0 \leq a<1\right)$; and $(g)$ follows by taking $\boldsymbol{m}=\mathbf{1}=$ $(1, \ldots, 1)$ by the symmetry of our random code design. Under our random code design and coset choice, for any $\boldsymbol{m}^{\prime} \neq \mathbf{1}$

$$
\begin{aligned}
& \operatorname{Pr}\left[\boldsymbol{D}_{\mathbf{1}}+\boldsymbol{V}=\boldsymbol{a}, \boldsymbol{D}_{\boldsymbol{m}^{\prime}}+\boldsymbol{V}=\boldsymbol{a}^{\prime}\right] \\
& =\sum_{\boldsymbol{v}} \operatorname{Pr}\left[\boldsymbol{V}=\boldsymbol{v}, \boldsymbol{D}_{\mathbf{1}}=\boldsymbol{a}-\boldsymbol{v}, \boldsymbol{D}_{\boldsymbol{m}^{\prime}}-\boldsymbol{D}_{\mathbf{1}}=\boldsymbol{a}^{\prime}-\boldsymbol{a}\right] \\
& =q^{-n K} \sum_{\boldsymbol{v}} \operatorname{Pr}\left[\boldsymbol{D}_{\mathbf{1}}=\boldsymbol{a}-\boldsymbol{v}, \boldsymbol{D}_{\boldsymbol{m}^{\prime}}-\boldsymbol{D}_{\mathbf{1}}=\boldsymbol{a}^{\prime}-\boldsymbol{a}\right] \\
& =q^{-n K} \operatorname{Pr}\left[\boldsymbol{D}_{\boldsymbol{m}^{\prime}}-\boldsymbol{D}_{\mathbf{1}}=\boldsymbol{a}^{\prime}-\boldsymbol{a}\right] \\
& \stackrel{(h)}{\leq} q^{-n K} \operatorname{Pr}\left[\boldsymbol{a}^{\prime}-\boldsymbol{a} \in \boldsymbol{D}\right] \\
& \cdot \operatorname{Pr}\left[\boldsymbol{D}_{\boldsymbol{m}^{\prime}}-\boldsymbol{D}_{\mathbf{1}}=\boldsymbol{a}^{\prime}-\boldsymbol{a} \mid \boldsymbol{a}^{\prime}-\boldsymbol{a} \in \boldsymbol{D}\right] \\
& \stackrel{(i)}{=} \quad q^{-n K} \frac{\bar{S}^{n}\left(\mathcal{T}_{\mathcal{Q}}^{n}\left(\boldsymbol{a}^{\prime}-\boldsymbol{a}\right)\right)}{B\left(n, \mathcal{T}_{\mathcal{Q}}^{n}\left(\boldsymbol{a}^{\prime}-\boldsymbol{a}\right)\right)} \frac{1}{M^{K}-1} \\
& \stackrel{(j)}{\leq} q^{-n K}\left(\alpha_{\text {мас }} q^{-n K}\right) \text {, }
\end{aligned}
$$

where $(h)$ follows since the difference between two codematrices is also a codematrix in any linear MAC code, and the upper bound continues to hold even when we select $M=q^{n R}$ codewords from the set of parity-check solutions; $(i)$ follows from the symmetry of our code design (since no variable node is treated any better or worse than any other variable node) and from our restriction to precisely $M=q^{n R}$ codewords in each single-transmitter codebook; and $(j)$ follows from the definition of $\alpha_{\text {мас }}$ in 22). Since $P_{\boldsymbol{D}_{1}+\boldsymbol{V}}(\boldsymbol{a})=q^{-n K}$ by the uniformity of random matrix $\boldsymbol{V}$,

$$
\operatorname{Pr}\left[\boldsymbol{D}_{\boldsymbol{m}^{\prime}}+\boldsymbol{V}=\boldsymbol{a}^{\prime} \mid \boldsymbol{D}_{\mathbf{1}}+\boldsymbol{V}=\boldsymbol{a}\right] \leq \alpha_{\mathrm{MAc}} q^{-n K} .
$$

Therefore

$$
\begin{aligned}
& E\left[\operatorname{Pr}\left[\mathcal{Y}_{\boldsymbol{M}, \boldsymbol{D}, \boldsymbol{V}}^{\mathrm{T}^{c}} \mid \boldsymbol{M}, \boldsymbol{D}, \boldsymbol{V}\right]\right] \\
& \leq \sum_{\boldsymbol{y}, \boldsymbol{a}} q^{-n K} P_{\boldsymbol{Y} \mid \boldsymbol{X}}(\boldsymbol{y} \mid \delta(\boldsymbol{a})) \\
& \cdot\left(\sum_{\boldsymbol{m}^{\prime}=\mathbf{2}}^{q^{n R K}} \sum_{\substack{\boldsymbol{a}^{\prime} \in \mathcal{Q}: \mathcal{T}_{\mathcal{Q}}^{n}\left(\boldsymbol{a}^{\prime}-\boldsymbol{a}\right) \in \mathrm{T}^{c} \\
P_{\boldsymbol{Y} \mid \boldsymbol{X}}\left(\boldsymbol{y} \mid \delta\left(\boldsymbol{a}^{\prime}\right)\right) \geq P_{\boldsymbol{Y} \mid \boldsymbol{X}}(\boldsymbol{y} \mid \delta(\boldsymbol{a}))}} \alpha_{\text {MA }} q^{-n K}\right)^{\rho} \\
& \leq \alpha_{\text {MAC }}^{\rho} \sum_{\boldsymbol{y}, \boldsymbol{a}} q^{-n K} P_{\boldsymbol{Y} \mid \boldsymbol{X}}(\boldsymbol{y} \mid \delta(\boldsymbol{a})) \\
& \cdot\left(\left(q^{n R K}-1\right) \sum_{\boldsymbol{a}^{\prime}: P_{\boldsymbol{Y} \mid \boldsymbol{X}}\left(\boldsymbol{y} \mid \delta\left(\boldsymbol{a}^{\prime}\right)\right) \geq P_{\boldsymbol{Y} \mid \boldsymbol{X}}(\boldsymbol{y} \mid \delta(\boldsymbol{a}))} q^{-n K}\right)^{\rho} \\
& \leq \alpha_{\mathrm{MAC}}^{\rho} q^{n R K \rho} \sum_{\boldsymbol{x}, \boldsymbol{y}} P_{\boldsymbol{Y} \mid \boldsymbol{X}}(\boldsymbol{y} \mid \boldsymbol{x}) \sum_{\boldsymbol{a}: \delta(\boldsymbol{a})=\boldsymbol{x}} q^{-n K} \\
& \cdot\left(\sum_{\boldsymbol{x}^{\prime}: P_{\boldsymbol{Y} \mid \boldsymbol{X}}\left(\boldsymbol{y} \mid \boldsymbol{x}^{\prime}\right) \geq P_{\boldsymbol{Y} \mid \boldsymbol{X}}(\boldsymbol{y} \mid \boldsymbol{x})} \sum_{\boldsymbol{a}^{\prime}: \delta\left(\boldsymbol{a}^{\prime}\right)=\boldsymbol{x}^{\prime}} q^{-n K}\right)^{\rho} \\
& =\alpha_{\text {MAC }}^{\rho} q^{n R K \rho} \sum_{\boldsymbol{x}, \boldsymbol{y}} P_{\boldsymbol{Y} \mid \boldsymbol{X}}(\boldsymbol{y} \mid \boldsymbol{x}) P_{\boldsymbol{X}}(\boldsymbol{x}) \\
& \cdot\left(\sum_{\boldsymbol{x}^{\prime}: P_{\boldsymbol{Y} \mid \boldsymbol{X}}\left(\boldsymbol{y} \mid \boldsymbol{x}^{\prime}\right) \geq P_{\boldsymbol{Y} \mid \boldsymbol{X}}(\boldsymbol{y} \mid \boldsymbol{x})} P_{\boldsymbol{X}}\left(\boldsymbol{x}^{\prime}\right)\right)^{\rho} \\
& \stackrel{(k)}{\leq} \alpha_{\mathrm{MAC}}^{\rho} q^{n R K \rho} \sum_{\boldsymbol{x}, \boldsymbol{y}} P_{\boldsymbol{Y} \mid \boldsymbol{X}}(\boldsymbol{y} \mid \boldsymbol{x}) P_{\boldsymbol{X}}(\boldsymbol{x}) \\
& \cdot\left(\sum_{\boldsymbol{x}^{\prime}} P_{\boldsymbol{X}}\left(\boldsymbol{x}^{\prime}\right)\left(\frac{P_{\boldsymbol{Y} \mid \boldsymbol{X}}\left(\boldsymbol{y} \mid \boldsymbol{x}^{\prime}\right)}{P_{\boldsymbol{Y} \mid \boldsymbol{X}}(\boldsymbol{y} \mid \boldsymbol{x})}\right)^{s}\right)^{\rho} \\
& =\alpha_{\mathrm{MAC}}^{\rho} q^{n R K \rho} \sum_{\boldsymbol{y}}\left(\sum_{\boldsymbol{x}} P_{\boldsymbol{X}}(\boldsymbol{x}) P_{\boldsymbol{Y} \mid \boldsymbol{X}}(\boldsymbol{y} \mid \boldsymbol{x})^{1-s \rho}\right) \\
& \cdot\left(\sum_{\boldsymbol{x}^{\prime}} P_{\boldsymbol{X}}\left(\boldsymbol{x}^{\prime}\right) P_{\boldsymbol{Y} \mid \boldsymbol{X}}\left(\boldsymbol{y} \mid \boldsymbol{x}^{\prime}\right)^{s}\right)^{\rho},
\end{aligned}
$$

where $(k)$ holds for any $s>0$.

When $s=1 /(1+\rho)$,

$$
\begin{aligned}
& E\left[\operatorname{Pr}\left[\mathcal{Y}_{M, \boldsymbol{D}, \boldsymbol{V}}^{\mathrm{T}^{c}} \mid \boldsymbol{M}, \boldsymbol{D}, \boldsymbol{V}\right]\right] \\
& \leq \alpha_{\mathrm{MAC}}^{\rho} q^{n R K \rho} \sum_{\boldsymbol{y}}\left(\sum_{\boldsymbol{x}} P_{\boldsymbol{X}}(\boldsymbol{x}) P_{\boldsymbol{Y} \mid \boldsymbol{X}}(\boldsymbol{y} \mid \boldsymbol{x})^{1 /(1+\rho)}\right)^{1+\rho} .
\end{aligned}
$$


Rewriting (232) in an exponential form using Gallager's error exponent gives the desired result .

\section{APPENDIX B}

TOOLS USED TO BOUND $\log \alpha_{\mathrm{MAC}} / n$ IN THEOREM 2

To bound the rate offset $\frac{\log \alpha_{\mathrm{MAC}}}{n}$, we first seek to understand how the normalized ensemble spectra (see Definition 9) for the MAC under the uniform random ensemble and the random LDPC code ensemble, here denoted by $\bar{S}_{U}(\boldsymbol{\theta})$ and $\bar{S}_{L}(\boldsymbol{\theta})$, respectively, differ. Lemma 3 first evaluates $\bar{S}_{U}(\boldsymbol{\theta})$. Theorem 17 then evaluates $\bar{S}_{L}(\boldsymbol{\theta})$. Theorem 18 relates $\bar{S}_{L}(\boldsymbol{\theta})$ to $\bar{S}_{U}(\boldsymbol{\theta})$ for a restricted family of pmfs $\boldsymbol{\theta}$, corresponding to codes in which the minimal distance is sufficiently large . Lemma 5 then paves the way for expurgation to remove codes with small minimal distance by showing that the probability of all codes with small minimum distance approaches zero as $n$ grows without bound under the proposed LDPC code ensemble.

Definition 9: (Normalized ensemble spectrum) Consider any ensemble of codes with ensemble-average spectrum $\bar{S}^{n}=$ $\left(\bar{S}^{n}(\boldsymbol{t}): \boldsymbol{t} \in \mathcal{T}_{\mathcal{Q}}^{n}\right)$. Given any rational pmf $\boldsymbol{\theta}=(\theta(g): g \in \mathcal{Q})$, let $\left\{n_{i}\right\}$ be a series of all indices $j$ such that $j \boldsymbol{\theta} \in \mathcal{T}_{\mathcal{Q}}^{j}$, the asymptotic exponent for $\boldsymbol{\theta}$ is defined by

$$
\bar{S}(\boldsymbol{\theta})=\lim _{i \rightarrow \infty} \frac{1}{n_{i}} \log \bar{S}^{n_{i}}\left(n_{i} \boldsymbol{\theta}\right),
$$

and the normalized ensemble spectrum for this ensemble is the collection of all asymptotic exponents $\bar{S}^{n}(\boldsymbol{\theta})$ for all pmfs $\boldsymbol{\theta}$.

Remark 18: For notational simplicity, we omit the index $i$ to write $\bar{S}^{n_{i}}\left(n_{i} \boldsymbol{\theta}\right)$ as $\bar{S}^{n}(n \boldsymbol{\theta})$ with the implicit assumption that $n \boldsymbol{\theta} \in \mathcal{T}_{\mathcal{Q}}^{n}$.

We consider the normalized ensemble spectrum for two ensembles, each with the same fixed rate $R=1-\frac{\lambda}{\rho} q$-ary symbols per channel use for each transmitter.

1) The first ensemble is an ensemble of uniform random $\mathrm{GF}(q) K$-transmitter MAC codes, where each transmitter employs a distinct blocklength- $n$ codebook with $q^{n R}$ codewords, $R=1-\frac{\lambda}{\rho}$, chosen uniformly at random from $\operatorname{GF}(q)^{n}$. We denote the normalized ensemble spectrum for this (uniform) random ensemble by

$$
\bar{S}_{U}(\boldsymbol{\theta}) \triangleq \lim _{n \rightarrow \infty} \frac{1}{n} \log \bar{S}_{U}^{n}(n \boldsymbol{\theta}),
$$

where $\overline{\boldsymbol{S}}_{U}$ (U stands for uniform) represents the ensemble-average spectrum under the $K$-MAC with independent codewords distributed uniformly on $\operatorname{GF}(q)^{n}$.

2) The second ensemble is the $\operatorname{LDPC}_{K}(\lambda, \rho ; n)$ ensemble. This is an ensemble of $K$-transmitter MAC codes for which all transmitters employ the same random codebook from the $\operatorname{LDPC}(\lambda, \rho ; n)$ ensemble. We denote the normalized ensemble spectrum for this LDPC code ensemble by

$$
\bar{S}_{L}(\boldsymbol{\theta}) \triangleq \lim _{n \rightarrow \infty} \frac{1}{n} \log \bar{S}_{L}^{n}(n \boldsymbol{\theta}),
$$

where $\overline{\boldsymbol{S}}_{L}$ ( $L$ stands for LDPC) represents the ensembleaverage spectrum under the $\operatorname{LDPC}_{K}(\lambda, \rho ; n)$ ensemble.

\section{A. Normalized Ensemble Spectrum for Uniform Random MAC} Ensemble

We begin by evaluating $\bar{S}_{U}(\boldsymbol{\theta})$.

Lemma 3: The normalized ensemble spectrum of the $K$ transmitter MAC uniform random ensemble is given by

$$
\bar{S}_{U}(\boldsymbol{\theta})=H(\boldsymbol{\theta})-K(1-R),
$$

where

$$
H(\boldsymbol{\theta})=-\sum_{g \in \mathcal{Q}} \theta(g) \log \theta(g)
$$

is the entropy of the pmf $\boldsymbol{\theta}$ in $q$-ary digits.

The proof of Lemma 3 is based on the discussion of binary codes in [31, Th. 1].

Proof: When each codeword is chosen uniformly at random from $\operatorname{GF}(q)^{n}$, the ensemble-average number $\bar{S}_{U}^{n}(n \boldsymbol{\theta})$ of codematrices of type $n \boldsymbol{\theta}$ is

$$
\begin{aligned}
\bar{S}_{U}^{n}(n \boldsymbol{\theta}) & =E_{U}\left[S_{\boldsymbol{D}}^{n}(n \boldsymbol{\theta})\right] \\
& =\sum_{\boldsymbol{m}} E_{U}\left[\mathbb{1}\left\{\mathcal{T}_{\mathcal{Q}}^{n}\left(\boldsymbol{D}_{\boldsymbol{m}}\right)=n \boldsymbol{\theta}\right\}\right] \\
& =q^{n R K} \operatorname{Pr}_{U}\left[\mathcal{T}_{\mathcal{Q}}^{n}\left(\boldsymbol{D}_{\mathbf{1}}\right)=n \boldsymbol{\theta}\right] \\
& =q^{n R K} \frac{B(n, n \boldsymbol{\theta})}{q^{n K}} .
\end{aligned}
$$

Applying the definition of the normalized ensemble spectrum gives

$$
\begin{aligned}
\bar{S}_{U}(\boldsymbol{\theta}) & \triangleq \lim _{n \rightarrow \infty} \frac{1}{n} \log \bar{S}_{U}^{n}(n \boldsymbol{\theta}) \\
& \stackrel{(a)}{=} \lim _{n \rightarrow \infty} \frac{1}{n} \log \left(q^{-n(1-R) K}\right)+H(\boldsymbol{\theta}) \\
& =H(\boldsymbol{\theta})-K(1-R),
\end{aligned}
$$

where $(a)$ follows from applying Stirling's upper and lower bounds on the factorial to the multinomial coefficient $B(n, n \boldsymbol{\theta})$. Note that the definition of $H(\boldsymbol{\theta})$ employs the base$q$ logarithm.

\section{B. Normalized Ensemble Spectrum for $\operatorname{LDPC}_{K}(\lambda, \rho ; n)$ En- semble}

Before moving on to the evaluating of $\bar{S}_{L}(\boldsymbol{\theta})$, recall that for any type $t \in \mathcal{T}_{\mathcal{Q}}^{\rho}, B(\rho, \boldsymbol{t})$ is the number of type- $\boldsymbol{t} \rho \times K$ matrices. For any type- $t$ matrix $G^{T}=\left[g_{1}^{T}, g_{2}^{T}, \ldots, g_{\rho}^{T}\right], g_{i} \in$ $\operatorname{GF}(q)^{K}$, let $G_{\boldsymbol{t}}$ be the corresponding matrix transpose, then

$$
\mathcal{N}_{\boldsymbol{t}}=\left|\left\{\boldsymbol{e} \in\{\mathrm{GF}(q) \backslash\{0\}\}^{\rho}: G_{\boldsymbol{t}} \boldsymbol{e}=\mathbf{0}\right\}\right|
$$

is the number of vectors $e \in\{\mathrm{GF}(q) \backslash\{0\}\}^{\rho}$ in the nullspace of $G_{t}$. Notice that $\mathcal{N}_{t}$ is constant across all matrices $G_{t}$ with type $t$. Theorem 17 employs this definition of $\mathcal{N}_{t}$ as well as the following notation. Given $x \in \mathbb{R}$,

$$
\operatorname{sgn}(x) \triangleq\left\{\begin{array}{cl}
1 & \text { if } x>0 \\
0 & \text { if } x=0 \\
-1 & \text { if } x<0
\end{array}\right.
$$

Note that the calculation of $\bar{S}_{L}^{n}$ in Theorem 17 is for the $\mathrm{LDPC}_{K}(\lambda, \rho ; n)$ ensemble before codeword removal. The true spectrum $\overline{\boldsymbol{S}}_{L}^{n}$ is smaller, and $\alpha_{\mathrm{MAC}}$ in 22) is a valid upper bound for the $\operatorname{LDPC}_{K}(\lambda, \rho ; n)$ ensemble (with codeword removal). 
Theorem 17: The normalized ensemble spectrum of the $\mathrm{LDPC}_{K}(\lambda, \rho ; n)$ ensemble is given by

$$
\bar{S}_{L}(\boldsymbol{\theta})=(1-\lambda) H(\boldsymbol{\theta})-\lambda \log (q-1)+\frac{\lambda}{\rho} \log \inf _{\substack{\boldsymbol{x}: \operatorname{sgn}(\boldsymbol{x}) \\=\operatorname{sgn}(\boldsymbol{\theta})}} \frac{A(\boldsymbol{x})}{\boldsymbol{x}^{\rho \boldsymbol{\theta}}},
$$

where for any pmf $\boldsymbol{x}=\left(x_{g}: g \in \mathcal{Q}\right)$ on $\mathcal{Q}$,

$$
\begin{aligned}
\boldsymbol{x}^{\rho \boldsymbol{\theta}} & =\prod_{g \in \mathcal{Q}} x_{g}^{d \theta(g)} \\
A(\boldsymbol{x}) & =\sum_{\boldsymbol{t} \in \mathcal{T}_{\mathcal{Q}}^{\rho}} \mathcal{N}_{\boldsymbol{t}} B(\rho, \boldsymbol{t}) \boldsymbol{x}^{\boldsymbol{t}} .
\end{aligned}
$$

Proof: Recall that when $n \boldsymbol{\theta}$ is a type, $\bar{S}_{L}(\boldsymbol{\theta})$ is the expected number of codematrices of type $n \boldsymbol{\theta}$ in a randomly drawn MAC codebook $\boldsymbol{D}$, corresponding to underlying single-transmitter $(\lambda, \rho ; n)$ LDPC code $\boldsymbol{C}$. Recall further that when $\boldsymbol{D}$ is the codebook of an LDPC MAC in $\operatorname{GF}(q)$, then $\overline{\boldsymbol{S}}\left(\left(n \theta_{g}: g \in\right.\right.$ $\mathcal{Q}))=\overline{\boldsymbol{S}}\left(\left(n \theta_{\pi(g)}: g \in \mathcal{Q}\right)\right)$ for any permutation $\pi$ on $[|\mathcal{Q}|]$.

Let $\mathcal{M}(n \boldsymbol{\theta})$ denote all possible codematrices of type $n \boldsymbol{\theta}$. Then

$$
\begin{aligned}
\bar{S}_{L}(n \boldsymbol{\theta}) & =E_{L}\left[\sum_{\boldsymbol{d}_{0} \in \mathcal{M}(n \boldsymbol{\theta})} \mathbb{1}\left\{\boldsymbol{d}_{0} \in \boldsymbol{D}\right\}\right] \\
& =\sum_{\boldsymbol{d}_{0} \in \mathcal{M}(n \boldsymbol{\theta})} E_{L}\left[\mathbb{1}\left\{\boldsymbol{d}_{0} \in \boldsymbol{D}\right\}\right] \\
& =\sum_{\boldsymbol{d}_{0} \in \mathcal{M}(n \boldsymbol{\theta})} \operatorname{Pr}\left[\boldsymbol{d}_{0} \in \boldsymbol{D}\right] \\
& =B(n, n \boldsymbol{\theta}) \operatorname{Pr}\left[\boldsymbol{d}_{n} \boldsymbol{\theta} \in \boldsymbol{D}\right],
\end{aligned}
$$

where $B(n, n \boldsymbol{\theta})$ is the size of $\mathcal{M}(n \boldsymbol{\theta}), \boldsymbol{d}_{n \boldsymbol{\theta}}$ is any fixed codematrix in $\mathcal{M}(n \boldsymbol{\theta})$, and the final equality follows from the symmetry of the code design. By the definition of $\bar{S}_{L}(\boldsymbol{\theta})$ in 235) and Stirling's upper and lower bounds on the factorial,

$$
\bar{S}_{L}(\boldsymbol{\theta})=H(\boldsymbol{\theta})+\lim _{n \rightarrow \infty} \frac{1}{n} \log \operatorname{Pr}\left[\boldsymbol{d}_{n \boldsymbol{\theta}} \in \boldsymbol{D}\right] .
$$

Remark 19: In the preceding characterization of $\overline{\boldsymbol{S}}_{L}(n \boldsymbol{\theta})$, $\operatorname{Pr}\left[\boldsymbol{d}_{n \boldsymbol{\theta}} \in \boldsymbol{D}\right]$ refers to the probability that $\boldsymbol{d}_{n \boldsymbol{\theta}}$ is in the codebook of a randomly drawn code $\boldsymbol{D}$ from the $\operatorname{LDPC}_{K}(\lambda, \rho ; n)$ ensemble. The calculation below evaluates this quantity by assuming that $\boldsymbol{D}$ is from the $\operatorname{LDPC}_{K}(\lambda, \rho ; n)$ ensemble without codeword removal, i.e., from the $\operatorname{LDPC}_{K}(\mathrm{Full}, \lambda, \rho ; n)$ ensemble. The true spectrum is smaller. Therefore, the resulting spectrum in 236 is a valid upper bound for $\operatorname{LDPC}_{K}(\lambda, \rho ; n)$ ensemble (with codeword removal),

To find $\operatorname{Pr}\left[\boldsymbol{d}_{n \boldsymbol{\theta}} \in \boldsymbol{D}\right]$, note that the random choice of edge connections and labels associates with each check node socket a socket value equal to the product of the edge value and the variable node value. There are $B(n \lambda, n \lambda \boldsymbol{\theta})$ equally likely assignments of variable node values to sockets that are consistent with PDF $\boldsymbol{\theta}$. Combining this with the $q-1$ possible labels for each edge, we find that there are

$$
t(\boldsymbol{\theta}, n)=B(n \lambda, n \lambda \boldsymbol{\theta})(q-1)^{n \lambda}
$$

equally likely outcomes for the choice of connections and edge values under a fixed codematrix $\boldsymbol{d}_{n \boldsymbol{\theta}}$. It is useful to note that some of these pairs yield the same socket values; for example, when a variable node holds value $\mathbf{0}$, the socket value is identical for all $q-1$ values of the edge. Since our probability calculation relies on a counting argument, the above value counts separately all events that yield the same output. This is different from the prior work [4, Eq. (49)], which counts the number of distinct outcomes rather than the number of distinct events leading to these outcomes in its probability calculation.

For $\boldsymbol{d}_{n \boldsymbol{\theta}}$ to be a codematrix, summing the $\rho$ (randomly chosen) socket values at each of the $n \lambda / \rho$ check nodes must give the value $0 \in \mathcal{Q}$. The following strategy and notation from [32, Sect. III.B] are useful in calculating the number of assignments that yield this outcome. First, for each fixed vector of edge values $\boldsymbol{e}=\left[e_{1}, \ldots, e_{\rho}\right] \in[q-1]^{\rho}$, we work to build a multinomial $f(\boldsymbol{x})$ in $\boldsymbol{x}=\left(x_{g}: g \in \mathcal{Q}\right)$ such that for any type $\boldsymbol{t}=(t(g): g \in \mathcal{Q}) \in \mathcal{T}_{\mathcal{Q}}^{n \lambda}$, the coefficient of the term $\boldsymbol{x}^{t}=\prod_{g \in \mathcal{Q}} x_{g}^{t(g)}$ equals the number of socket assignment and edge value pairs for which the socket assignment carries variable node values of type $t$, and the socket values satisfy all check node constraints? Then, using notation $\lfloor f(\boldsymbol{x})\rfloor_{t}$ to designate a function that maps multinomial $f(\boldsymbol{x})$ to the coefficient of element $\boldsymbol{x}^{t}$, we extract the number of socket and edge value assignments that are consistent with the fixed codematrix $\boldsymbol{d}_{n \boldsymbol{\theta}}$ and satisfy all constraint nodes; this is the number of randomly designed codes for which $\boldsymbol{d}_{n \boldsymbol{\theta}}$ is a codematrix.

To begin, consider a single check node. Let $g_{1}, \ldots, g_{\rho}$ denote the values at the $\rho$ variable nodes connected to that check node, and let $e_{1}, \ldots, e_{\rho}$ be the corresponding edge values. We seek to build a multinomial $A(\boldsymbol{x})$ in which the coefficient of each term $\boldsymbol{x}^{t}$ is the number of distinct edge value and socket assignments for which the variable-node inputs have type $t$ and the check node is satisfied. That is,

$$
\begin{aligned}
A(\boldsymbol{x}) & \sum_{g_{1}, \ldots, g_{\rho} \in \mathcal{Q}} \sum_{e_{1}, \ldots, e_{\rho} \in[q-1]} \mathbb{1}\left\{\sum_{i=1}^{\rho} e_{i} g_{i}=\mathbf{0}\right\}\left(\prod_{i=1}^{\rho} x_{g_{i}}\right) \\
& =\sum_{e_{1}, \ldots, e_{\rho} \in[q-1]} \sum_{\hat{g}_{1}, \ldots, \hat{g}_{\rho} \in \mathcal{Q}} \mathbb{1}\left\{\sum_{i=1}^{\rho} \hat{g}_{i}=\mathbf{0}\right\}\left(\prod_{i=1}^{\rho} x_{\hat{g}_{i} / e_{i}}\right) .
\end{aligned}
$$

Note that the above expression implements the multiplication $e_{i} g_{i}$ by viewing $g_{i}$ as length- $K$ vector over $\operatorname{GF}(q)$, and similarly for the division $\hat{g}_{i} / e_{i}$. Recall that $q$ is a prime power, say $q=p^{m}$, and that $\mathcal{Q}=\mathrm{GF}(q)^{K}$. We can therefore view each element $g \in \mathcal{Q}$ as a corresponding vector $\boldsymbol{h} \in$ $\{0, \ldots, p-1\}^{m K}$ and implement addition in $\mathcal{Q}$ as componentwise addition modulo- $p$. Thus, following the argument of [4] Theorem 8], for each fixed value of $\left(e_{1}, \ldots, e_{\rho}\right)$, the given sum equals a $\rho$-fold, $K m$-dimensional cyclic convolution evaluated at $\mathbf{0}$, giving

$$
\begin{aligned}
& A(\boldsymbol{x}) \\
& =\sum_{\boldsymbol{e}_{1}, \ldots, \boldsymbol{e}_{\rho} \in \mathrm{GF}(p)^{m} \backslash\{\mathbf{0}\}} \sum_{\substack{\boldsymbol{h}_{1}, \ldots, \boldsymbol{h}_{\rho} \in \mathrm{GF}(p)^{m K} \\
\sum_{i=1}^{\rho} \boldsymbol{h}_{i}=\mathbf{0}}}\left(\prod_{i=1}^{\rho} x_{\frac{\boldsymbol{h}_{i}}{\boldsymbol{e}_{i}}}\right)
\end{aligned}
$$

${ }^{1}$ The type is with respect to vectors of length $n \lambda$ since each of $n$ variable nodes is employed in $\lambda$ sockets, giving a total of $n \lambda$ socket values. 


$$
\begin{aligned}
& =\sum_{\boldsymbol{e}_{1}, \ldots, \boldsymbol{e}_{\rho} \in \operatorname{GF}(p)^{m} \backslash\{\mathbf{0}\}}\left[x\left[\frac{\boldsymbol{h}}{\boldsymbol{e}_{1}}\right] * \cdots * x\left[\frac{\boldsymbol{h}}{\boldsymbol{e}_{\rho}}\right]\right]_{\boldsymbol{h}=\mathbf{0}} \\
& =\sum_{\substack{\boldsymbol{e}_{1}, \ldots, \boldsymbol{e}_{\rho} \in \\
\mathrm{GF}(p)^{m} \backslash\{\mathbf{0}\}}}\left[\operatorname{IDFT}\left[\prod_{j=1}^{\rho} \operatorname{DFT}\left[x\left[\frac{\boldsymbol{h}}{\boldsymbol{e}_{j}}\right]\right]\right]\right]_{\boldsymbol{h}=\mathbf{0}} \\
& =\sum_{\substack{\boldsymbol{e}_{1}, \ldots, \boldsymbol{e}_{\rho} \in \\
\mathrm{GF}(p)^{m} \backslash\{\mathbf{0}\}}} \frac{1}{q^{K}} \sum_{\boldsymbol{k} \in \mathrm{GF}(p)^{m K}}\left[\prod_{i=1}^{\rho}\right. \\
& \left.\left(\sum_{\boldsymbol{h} \in \mathrm{GF}(p)^{m K}} e^{-j \frac{2 \pi}{p} \sum_{\ell=0}^{m K} k_{\ell} h_{\ell}} x\left[\frac{\boldsymbol{h}}{\boldsymbol{e}_{i}}\right]\right)\right],
\end{aligned}
$$

where for any $\boldsymbol{h} \in\{0, \ldots, p-1\}^{m K}, x[\boldsymbol{h}]$ equals $x_{g}$ for the corresponding $g \in \mathcal{Q}$.

Combining $n \lambda / \rho$ such multinomials, corresponding to our $n \lambda / \rho$ check nodes, gives multinomial $(A(\boldsymbol{x}))^{n \lambda / \rho}$. The coefficient of the term $\boldsymbol{x}^{n \lambda \boldsymbol{\theta}}$ in this multinomial describes the number of edge and socket assignments for which $\boldsymbol{d}_{n \theta}$ is a codematrix. We denote this number by

$$
e(\boldsymbol{\theta}, n)=\left\lfloor\left((A(\boldsymbol{x}))^{n \lambda / \rho}\right)\right\rfloor_{n \lambda \boldsymbol{\theta}} .
$$

Applying this definition, we have

$$
\begin{aligned}
\lim _{n \rightarrow \infty} & \frac{1}{n} \log \operatorname{Pr}\left[\boldsymbol{d}_{n \boldsymbol{\theta}} \in \boldsymbol{D}\right] \\
= & \lim _{n \rightarrow \infty} \frac{1}{n} \log \frac{e(\boldsymbol{\theta}, n)}{t(\boldsymbol{\theta}, n)} \\
= & \lim _{n \rightarrow \infty} \frac{1}{n} \log \frac{\left\lfloor\left((A(\boldsymbol{x}))^{n \lambda / \rho}\right)\right\rfloor_{n \lambda \boldsymbol{\theta}}}{B(n \lambda, n \lambda \boldsymbol{\theta})(q-1)^{n \lambda}} \\
= & -\lambda H(\boldsymbol{\theta})-\lambda \log (q-1) \\
& +\frac{\lambda}{\rho} \lim _{\frac{\lambda}{\rho} n \rightarrow \infty} \frac{1}{\frac{\lambda}{\rho} n} \log \left\lfloor(A(\boldsymbol{x}))^{\frac{\lambda}{\rho} n}\right\rfloor_{\left(\frac{\lambda}{\rho} n\right) \rho \boldsymbol{\theta}} \\
= & -\lambda H(\boldsymbol{\theta})-\lambda \log (q-1) \\
& +\frac{\lambda}{\rho} \lim _{n \rightarrow \infty} \frac{1}{n} \log \left\lfloor(A(\boldsymbol{x}))^{n}\right\rfloor_{n \rho \boldsymbol{\theta}} \\
\stackrel{(a)}{=} & -\lambda H(\boldsymbol{\theta})-\lambda \log (q-1)+\frac{\lambda}{\rho} \log _{\substack{\boldsymbol{x}: \operatorname{sgn}(\boldsymbol{x}) \\
=\operatorname{sgn}(\boldsymbol{\theta})}} \frac{A(\boldsymbol{x})}{\prod_{g} x_{g}^{\rho \theta_{g}},}
\end{aligned}
$$

where (a) follows from the definition of $\lfloor A(\boldsymbol{x})\rfloor_{t}$ as the coefficient of element $\boldsymbol{x}^{t}$ in multinomial $A(\boldsymbol{x})$ and from the second equation in [4, Theorem 10] (included below for reference), which gives an expression for evaluating the limit of multinomial coefficient exponent $\frac{1}{n} \log \left\lfloor(A(\boldsymbol{x}))^{n}\right\rfloor_{n \rho \boldsymbol{\theta}}$. Combining the given limit with 237) gives

$$
\bar{S}_{L}(\boldsymbol{\theta})=(1-\lambda) H(\boldsymbol{\theta})-\lambda \log (q-1)+\frac{\lambda}{\rho} \log \inf _{\substack{\boldsymbol{x}: \operatorname{sgn}(\boldsymbol{x}) \\=\operatorname{sgn}(\boldsymbol{\theta})}} \frac{A(\boldsymbol{x})}{\boldsymbol{x}^{\rho \boldsymbol{\theta}}},
$$

which is the desired result.

Lemma 4: ([4, Th. 10]). Let $\gamma>0$ be some rational number and $p(x, y)^{\gamma}$ be a multinomial with non-negative coefficient. Let $\alpha>0$ and $\beta>0$ be rational numbers, and $\left\{n_{i}\right\}$ be a series of all indices $j$ such that $j / \gamma \in \mathbb{Z},\left\lfloor p(x, y)^{j}\right\rfloor_{\alpha j, \beta j} \neq 0$, then

$$
\left\lfloor p(x, y)^{n_{i}}\right\rfloor_{\alpha n_{i}, \beta n_{i}} \leq \inf _{x>0, y>0} \frac{p(x, y)^{n_{i}}}{x^{\alpha n_{i}} y^{\beta n_{i}}}
$$

and

$$
\lim _{i \rightarrow \infty} \frac{1}{n_{i}} \log \left\lfloor p(x, y)^{n_{i}}\right\rfloor_{\alpha n_{i}, \beta n_{i}}=\log \inf _{x>0, y>0} \frac{p(x, y)}{x^{\alpha} y^{\beta}} .
$$

\section{Relationship between $\bar{S}_{U}(\boldsymbol{\theta})$ and $\bar{S}_{L}(\boldsymbol{\theta})$}

Rather than comparing $\bar{S}_{U}(\boldsymbol{\theta})$ and $\bar{S}_{L}(\boldsymbol{\theta})$ for all possible values of $\theta$, Theorem 18 , below, makes this comparison only for pmfs $\boldsymbol{\theta}$ that lie in a restricted family of pmfs $J_{\sigma}$ on alphabet $\mathcal{Q}$. We begin by defining this family. For any $\sigma \in(0,1), J_{\sigma}$ eliminates all pmfs with $\theta(\mathbf{0})$ above $1-\sigma$; precisely,

$$
\begin{aligned}
& J_{\sigma}=\left\{\left(\boldsymbol{\theta}=(\theta(g): g \in \mathcal{Q}): \sum_{g \in \mathcal{Q}} \theta(g)=1,\right.\right. \\
&0 \leq \theta(\mathbf{0}) \leq 1-\sigma, \theta(g) \geq 0 \forall g \in \mathcal{Q} \backslash\{\mathbf{0}\}\},
\end{aligned}
$$

where $\mathbf{0}$ is the all zero vector.

By an argument similar to that used for LDPC codes on the PPC in [4], Theorem 18 shows uniform convergence of $\bar{S}_{L}(\boldsymbol{\theta})$ to $\bar{S}_{U}(\boldsymbol{\theta})$ for the subset of values of $\boldsymbol{\theta} \in J_{\sigma}$.

Theorem 18: For any positive rational number $R<1$, any $\sigma \in(0,1)$, and any $\epsilon>0$, there exists a constant $\rho_{0}>0$ such that for all $\boldsymbol{\theta} \in J_{\sigma}$, and all $\lambda, \rho$ for which $R=1-\frac{\lambda}{\rho}$ and $\rho>\rho_{0}$,

$$
\bar{S}_{L}(\boldsymbol{\theta})<\bar{S}_{U}(\boldsymbol{\theta})+\epsilon .
$$

Proof: To prove $\bar{S}_{L}(\boldsymbol{\theta})<\bar{S}_{U}(\boldsymbol{\theta})+\epsilon$ for large enough $\rho$ and $\boldsymbol{\theta} \in J_{\sigma}$, we derive an upper bound on the limit of $\bar{S}_{L}(\boldsymbol{\theta})$ as $\rho$ approaches $n$ and show that the upper bound equals $\bar{S}_{U}(\boldsymbol{\theta})$.

We start with the expression of $\bar{S}_{L}(\boldsymbol{\theta})$ from Theorem 17

$$
\begin{aligned}
\bar{S}_{L}(\boldsymbol{\theta}) & =(1-\lambda) H(\boldsymbol{\theta})-\lambda \log (q-1)+\frac{\lambda}{\rho} \log \inf _{\substack{\boldsymbol{x}: \operatorname{sgn}(\boldsymbol{x}) \\
=\operatorname{sgn}(\boldsymbol{\theta})}} \frac{A(\boldsymbol{x})}{\boldsymbol{x}^{\rho \boldsymbol{\theta}}} \\
& \leq(1-\lambda) H(\boldsymbol{\theta})-\lambda \log (q-1)+\frac{\lambda}{\rho} \log \frac{A(\boldsymbol{\theta})}{\boldsymbol{\theta}^{\rho \boldsymbol{\theta}}} \\
& =H(\boldsymbol{\theta})-\lambda \log (q-1)+\frac{\lambda}{\rho} \log A(\boldsymbol{\theta}) .
\end{aligned}
$$

We next focus on $A(\boldsymbol{\theta})$ to bound the last term in this equation.

Given our equation for $A(\boldsymbol{x})$ from (238), it follows that

$$
\begin{aligned}
A(\boldsymbol{x})= & \sum_{\substack{\boldsymbol{e}_{1}, \ldots, \boldsymbol{e}_{\rho} \in \\
\mathrm{GF}(p)^{m} \backslash\{\mathbf{0}\}}} \frac{1}{q^{K}} \sum_{\boldsymbol{k} \in \mathrm{GF}(p)^{m K}} \\
& \prod_{i=1}^{\rho}\left(\sum_{\boldsymbol{h} \in \mathrm{GF}(p)^{m K}} e^{-j \frac{2 \pi}{p} \sum_{\ell=0}^{m K} k_{\ell} h_{\ell}} x\left[\frac{\boldsymbol{h}}{\boldsymbol{e}_{i}}\right]\right) \\
& \stackrel{(a)}{=} \frac{(q-1)^{\rho}}{q^{K}}+\frac{1}{q^{K}} \sum_{\boldsymbol{k} \in \mathrm{GF}(p)^{m K} \backslash\{\mathbf{0}\}}\left(\sum_{\boldsymbol{e} \in \mathrm{GF}(p)^{m} \backslash\{\mathbf{0}\}}\right. \\
& \left.\left(\sum_{\boldsymbol{h} \in \mathrm{GF}(p)^{m K}} e^{-j \frac{2 \pi}{p} \sum_{\ell=0}^{m K} k_{\ell} h_{\ell} x}\left[\frac{\boldsymbol{h}}{\boldsymbol{e}}\right]\right)\right)^{\rho} \\
\stackrel{(b)}{=} & \frac{(q-1)^{\rho}}{q^{K}}+\frac{1}{q^{K}} \sum_{\boldsymbol{k} \in \mathrm{GF}(p)^{m K} \backslash\{\mathbf{0}\}}\left(\sum_{\boldsymbol{h} \in \mathrm{GF}(p)^{m K}}\right.
\end{aligned}
$$




$$
\begin{aligned}
& \left.\cdot e^{-j \frac{2 \pi}{p} \sum_{\ell=0}^{m K} k_{\ell} h_{\ell}}\left(\sum_{\boldsymbol{e} \in \operatorname{GF}(p)^{m} \backslash\{\mathbf{0}\}} x\left[\frac{\boldsymbol{h}}{\boldsymbol{e}}\right]\right)\right)^{\rho} \\
& \stackrel{(c)}{=} \frac{(q-1)^{\rho}}{q^{K}}+\frac{1}{q^{K}} \sum_{\boldsymbol{k} \in \mathrm{GF}(p)^{m K} \backslash\{\mathbf{0}\}}(x[\mathbf{0}](q-1) \\
& \left.+(1-x[\mathbf{0}]) \sum_{\substack{\boldsymbol{h} \in \\
\operatorname{GF}(p)^{m K} \backslash\{\mathbf{0}\}}} e^{-j \frac{2 \pi}{p} \sum_{\ell=0}^{m K} k_{\ell} h_{\ell}}\right)^{\rho} \\
& \stackrel{(d)}{=} \frac{(q-1)^{\rho}}{q^{K}}+\frac{(q-1)^{\rho}}{q^{K}}\left(\sum_{\boldsymbol{k} \neq \mathbf{0}}(x[\mathbf{0}]+(1-x[\mathbf{0}])\right. \\
& \left.\left.\cdot \frac{\sum_{\boldsymbol{h} \neq \mathbf{0}} e^{-j \frac{2 \pi}{p} \sum_{\ell=0}^{m K} k_{\ell} h_{\ell}}}{q-1}\right)^{\rho}\right) \text {, }
\end{aligned}
$$

where $(a)$ follows by first separating the term $\boldsymbol{k}=\mathbf{0}$ and noting $\sum_{g \in \mathcal{Q}} \boldsymbol{x}[g]=1$, then interchanging the order of summation, and noting that the product term is identical for all $\boldsymbol{e}_{i}, i \in[\rho] ;(b)$ holds since the exponential term is independent of $\boldsymbol{e} ;(c)$ follows by separating the summation over $\boldsymbol{h} \in \operatorname{GF}(q)^{m K}$ into the case where $\boldsymbol{h}=\mathbf{0}$ and the case where $\boldsymbol{h} \neq \mathbf{0} ;(d)$ follows from taking a factor of $(q-1)^{\rho}$ out of the summation over $\boldsymbol{k}$.

To bound the final term, notice that for each $\boldsymbol{k} \in \mathrm{GF}(p)^{m K}$

$$
\begin{aligned}
& \left|\frac{1}{q-1} \sum_{\boldsymbol{h} \neq \mathbf{0}} e^{-j \frac{2 \pi}{p} \sum_{\ell=0}^{m K} k_{\ell} h_{\ell}}\right|^{2} \\
& =\left|\frac{1}{q-1} \sum_{\hat{p}=0}^{p-1} \sum_{\boldsymbol{h} \neq \mathbf{0}: \sum_{\ell} k_{\ell} h_{\ell}=\hat{p}} e^{-j \frac{2 \pi}{p} \hat{p}}\right|^{2} \\
& \stackrel{(e)}{\leq} \max _{\hat{p} \in[p-1]} \mid \frac{\left|\left\{\boldsymbol{h} \neq \mathbf{0}: \sum_{\ell} k_{\ell} h_{\ell}=0\right\}\right|}{q-1} \\
& \quad+\left.\frac{\left|\left\{\boldsymbol{h} \neq \mathbf{0}: \sum_{\ell} k_{\ell} h_{\ell} \neq 0\right\}\right|}{q-1} e^{-j \frac{2 \pi}{p} \hat{p}}\right|^{2} \\
& =\max _{\hat{p} \in[p-1]}\left|\left(1-\lambda_{\boldsymbol{k}}\right)+\lambda_{\boldsymbol{k}} e^{-j \frac{2 \pi}{p} \hat{p}}\right|^{2},
\end{aligned}
$$

where $(e)$ holds by separating the summation over $\boldsymbol{h} \neq 0$ for which $\sum_{l} k_{l} h_{l}=\hat{p}$ into cases where $\hat{p}=0$ and the cases where $\hat{p} \neq 0$ and using the maximum term for the $\hat{p} \neq 0$ group; finally, in the last term, we let

$$
\lambda_{\boldsymbol{k}}=\frac{\left|\left\{\boldsymbol{h} \neq \mathbf{0}: \sum_{\ell} k_{\ell} h_{\ell} \neq 0\right\}\right|}{q-1} ;
$$

notice that $\lambda_{k}$ is a function of $\boldsymbol{k}$ and $q$.

Therefore, setting

$$
\tau \triangleq \max _{\hat{p} \in[q-1]} \operatorname{Re}\left(e^{-j \frac{2 \pi \hat{p}}{p}}\right)
$$

gives the following upper bound

$$
\left|\frac{1}{q-1} \sum_{\boldsymbol{h} \neq \mathbf{0}} e^{-j \frac{2 \pi}{p} \sum_{\ell=0}^{m K} k_{\ell} h_{\ell}}\right|^{2}
$$

$$
\begin{aligned}
& \leq \max _{\hat{p} \in[p-1]}\left[\left(1-\lambda_{\boldsymbol{k}}\right)^{2}+\lambda_{\boldsymbol{k}}^{2}+2 \lambda_{\boldsymbol{k}}\left(1-\lambda_{\boldsymbol{k}}\right) \operatorname{Re}\left(e^{-j \frac{2 \pi \hat{p}}{p}}\right)\right] \\
& =\left(1-\lambda_{\boldsymbol{k}}\right)^{2}+\lambda_{\boldsymbol{k}}^{2}+2 \tau \lambda_{\boldsymbol{k}}\left(1-\lambda_{\boldsymbol{k}}\right) \\
& =1-(1-\tau) 2 \lambda_{\boldsymbol{k}}\left(1-\lambda_{\boldsymbol{k}}\right) \\
& \leq \psi^{2},
\end{aligned}
$$

where

$$
\psi^{2} \triangleq \max _{\boldsymbol{k} \neq \mathbf{0}}\left[1-(1-\tau) 2 \lambda_{\boldsymbol{k}}\left(1-\lambda_{\boldsymbol{k}}\right)\right] .
$$

Notice that $\psi^{2}$ depends on $q$ but does not vary with $\boldsymbol{x}$. Notice further that $\psi^{2}$ lies in $(0,1)$ since $\tau \in(0,1)$ for all $q$ and $\lambda_{\boldsymbol{k}} \in(0,1)$ for all $\boldsymbol{k} \neq \mathbf{0}$; therefore, $2 \lambda_{k}\left(1-\lambda_{k}\right) \in\left(0, \frac{1}{2}\right]$. Noting that $x[\mathbf{0}] \in[0,1-\sigma]$ by assumption $\left(\boldsymbol{x} \in J_{\sigma}\right)$, we have

$$
\begin{aligned}
&\left|x[\mathbf{0}]+(1-x[\mathbf{0}]) \frac{\sum_{\boldsymbol{h} \neq \mathbf{0}} e^{-j \frac{2 \pi}{p} \sum_{\ell=0}^{m K} k_{\ell} h_{\ell}}}{q-1}\right|^{2} \\
& \leq(x[\mathbf{0}]+\psi(1-x[\mathbf{0}]))^{2}<1,
\end{aligned}
$$

Taking the square root of both sides gives

$$
\begin{array}{r}
\left|x[\mathbf{0}]+(1-x[\mathbf{0}]) \frac{\sum_{\boldsymbol{h} \neq \mathbf{0}} e^{-j \frac{2 \pi}{p} \sum_{\ell=0}^{m K} k_{\ell} h_{\ell}}}{q-1}\right| \\
\leq(x[\mathbf{0}]+\psi(1-x[\mathbf{0}])) .
\end{array}
$$

Returning to our equation for $A(\boldsymbol{x})$ in 245, we have

$$
\frac{\left|A(\boldsymbol{x})-\frac{(q-1)^{\rho}}{q^{K}}\right|}{\frac{(q-1)^{\rho}}{q^{K}}} \leq\left|\sum_{\boldsymbol{k} \neq \mathbf{0}}(x[\mathbf{0}]+\psi(1-x[\mathbf{0}]))^{\rho}\right|,
$$

which gives an upper bound on $A(\boldsymbol{x})$

$$
A(\boldsymbol{x}) \leq \frac{(q-1)^{\rho}}{q^{K}}\left(1+\sum_{\boldsymbol{k} \neq \mathbf{0}}(x[\mathbf{0}]+\psi(1-x[\mathbf{0}]))^{\rho}\right) .
$$

Therefore, we obtain

$$
\begin{aligned}
\log A(\boldsymbol{\theta}) \leq & \log \left(\frac{(q-1)^{\rho}}{q^{K}}\right) \\
& +\log \left(1+\sum_{\boldsymbol{k} \neq \mathbf{0}}(\boldsymbol{\theta}[\mathbf{0}]+\psi(1-\boldsymbol{\theta}[\mathbf{0}]))^{\rho}\right)
\end{aligned}
$$

where the second term approaches 0 as $\rho$ increases uniformly for all $\boldsymbol{\theta} \in J_{\delta}$. Returning to (244), fixing $\lambda=\rho(1-R)$ and letting $\rho=\kappa n$ for some constant $\kappa$ gives

$$
\begin{aligned}
\lim _{n \rightarrow \infty} \bar{S}_{L}(\boldsymbol{\theta}) & \\
\leq & \lim _{n \rightarrow \infty}\left[H(\boldsymbol{\theta})-\lambda \log (q-1)+\frac{\lambda}{\rho} \log \left(\frac{(q-1)^{\rho}}{q^{K}}\right)\right. \\
& \left.\quad+\frac{\lambda}{\rho} \log \left(1+\sum_{\boldsymbol{k} \neq \mathbf{0}}(\boldsymbol{\theta}[\mathbf{0}]+\psi(1-\boldsymbol{\theta}[\mathbf{0}]))^{\rho}\right)\right] \\
=H(\boldsymbol{\theta})- & \frac{\lambda}{\rho} K \\
+\lim _{n \rightarrow \infty} & {\left[\frac{\lambda}{\rho} \log \left(1+\sum_{\boldsymbol{k} \neq \mathbf{0}}(\boldsymbol{\theta}[\mathbf{0}]+\psi(1-\boldsymbol{\theta}[\mathbf{0}]))^{\kappa n}\right)\right] } \\
= & H(\boldsymbol{\theta})-K(1-R)+0
\end{aligned}
$$




$$
=\bar{S}_{U}(\boldsymbol{\theta}) .
$$

Note that the choice of $\kappa$ in $\rho=\kappa n$ should be much smaller than $\frac{q-1}{q}$ to maintain some of the sparsity of LDPC codes. The upper bound $\frac{q-1}{q}$ is chosen to ensure that the edges values in the Tanner graph can be chosen from $\operatorname{GF}(q) \backslash\{0\}$ instead of $\operatorname{GF}(q)$.

If, instead of setting $\rho=\kappa n$ for some constant $\kappa$, we set $\rho=\kappa(n) n$ for some function $\kappa(n)$ that satisfies $\kappa(n) n \rightarrow \infty$ as $n \rightarrow \infty$, we again find that $\lim _{n \rightarrow \infty} \bar{S}_{L}(\boldsymbol{\theta})=\bar{S}_{U}(\boldsymbol{\theta})$. In Appendix D, we show that in order for $\frac{1}{n} \log \alpha_{\text {MAC }}$ to behave as $O\left(\frac{\log n}{n}\right), \kappa(n)$ should decay no more quickly than $\Theta\left(\frac{\log n}{n}\right)$.

\section{APPENDIX C}

\section{Probability of Small Minimum Distance Codes in} $\mathrm{THE}^{\mathrm{LDPC}} \mathrm{CP}_{K}(\lambda, \rho ; n)$ ENSEMBLE

Since Theorem 18 bounds the difference between $\bar{S}_{L}(\boldsymbol{\theta})$ and $\bar{S}_{U}(\boldsymbol{\theta})$ only when $\boldsymbol{\theta} \in J_{\sigma}$, it does not eliminate the possibility that $\alpha_{\text {мас }}$ (defined in (22) ) may be large for all values of $\lambda$ and $\rho$ if we consider all possible values of $\boldsymbol{\theta}$.

To resolve this problem, Theorem 2 removes from the $\mathrm{LDPC}_{K}($ Full, $\lambda, \rho ; n)$ ensemble all codes for which the minimum distance between codematrices is less than or equal to $\gamma n$. Recall that the distance between two codematrices $\boldsymbol{d}_{1}$ and $\boldsymbol{d}_{2}$ with dimension $n \times K$ is the number of rows they differ,

$$
d\left(\boldsymbol{d}_{1}, \boldsymbol{d}_{2}\right)=\sum_{i=1}^{n} \mathbb{1}\left(\boldsymbol{d}_{1}[i, *] \neq \boldsymbol{d}_{2}[i, *]\right) ;
$$

that is, $d\left(\boldsymbol{d}_{1}, \boldsymbol{d}_{2}\right)$ is the number of time slots in which the transmissions for codematrices $\boldsymbol{d}_{1}$ and $\boldsymbol{d}_{2}$ differ. The minimum distance of codebook $\boldsymbol{d}$ is

$$
d_{\min }(\boldsymbol{d})=\min _{\boldsymbol{m} \neq \boldsymbol{m}^{\prime}} d\left(\boldsymbol{d}_{\boldsymbol{m}}, \boldsymbol{d}_{\boldsymbol{m}^{\prime}}\right) .
$$

In [4. Th. 6], Bennatan et al. prove that if $C$ is a randomly chosen code from the LDPC(Full, $\lambda, \rho ; n)$ ensemble (using the full collection of legitimate codewords, rather than our possibly reduced collection of codewords), then there exists some $\gamma \in(0,1 / 2]$ that depends only on $R$ and $q$ such that

$$
\operatorname{Pr}\left[d_{\min }(\boldsymbol{C}) \leq \gamma n\right]=O\left(n^{-\left(\frac{\lambda}{2}-1\right)}\right),
$$

where the distance between two codewords is the number of positions at which the corresponding symbols are different.

Lemma 5 builds on this result in order to bound the probability of codes with small minimum distance under the random LDPC code design. This bound is later employed in the proof of Theorem 2 (see Appendix D to bound the change in ensemble-average number of codematrices due to expurgation.

Lemma 5: Fix the rate $R=1-\frac{\lambda}{\rho}$. Let $\lambda \geq 3$ and fix some prime power $q$. Let $\boldsymbol{D}$ be a randomly chosen code from the $\operatorname{LDPC}_{K}(\lambda, \rho ; n)$ ensemble. Then there exists some $\gamma \in$ $(0,1 / 2]$ that depends only on $R$ and $q$ such that

$$
\operatorname{Pr}\left[d_{\min }(\boldsymbol{D}) \leq \gamma n\right]=O\left(n^{-\left(\frac{\lambda}{2}-1\right)}\right) .
$$

Proof: We begin by noting that Bennatan et al.'s singletransmitter bound (248) on the minimum distance continues to hold if one restricts code $C$ to exactly $q^{n R}$ codewords through random codeword selection; this follows because removing codewords from the codebook cannot decrease the pairwise minimum distance between the codewords that remain.

We next show that $d_{\min }(\boldsymbol{D})=d_{\min }(\boldsymbol{C})$, where $\boldsymbol{C}$ is the underlying single-transmitter code for $\boldsymbol{D}$.

First note that $d_{\min }(\boldsymbol{D}) \leq d\left(\boldsymbol{D}_{\boldsymbol{m}}, \boldsymbol{D}_{\boldsymbol{m}^{\prime}}\right)$, where $\boldsymbol{m}$ and $\boldsymbol{m}^{\prime}$ are any pair of index vectors from $[M]^{K}$ that differ in exactly one component. Choosing the element in that differing component to be any pair $(i, j)$ for which $d\left(\boldsymbol{C}_{i}, \boldsymbol{C}_{j}\right)=d_{\min }(\boldsymbol{C})$ shows that $d_{\min }(\boldsymbol{D}) \leq d_{\min }(\boldsymbol{C})$; that is, since $\boldsymbol{m}$ and $\boldsymbol{m}^{\prime}$ differ in exactly one component, say $\boldsymbol{m}=(i, 1, \ldots, 1)$ and $\boldsymbol{m}^{\prime}=(j, 1, \ldots, 1)$, the time slots in which $\boldsymbol{D}_{\boldsymbol{m}}$ and $\boldsymbol{D}_{\boldsymbol{m}^{\prime}}$ differ are exactly the time slots in which $\boldsymbol{C}_{i}$ and $\boldsymbol{C}_{j}$ differ, giving

$$
d_{\min }(\boldsymbol{D}) \leq d\left(\boldsymbol{D}_{\boldsymbol{m}}, \boldsymbol{D}_{\boldsymbol{m}^{\prime}}\right)=d\left(\boldsymbol{C}_{i}, \boldsymbol{C}_{j}\right)=d_{\min }(\boldsymbol{C}) .
$$

To prove that this bound is tight, note that the distance $d\left(\boldsymbol{D}_{\boldsymbol{m}}, \boldsymbol{D}_{\boldsymbol{m}^{\prime}}\right)$ between any pair of distinct codematrices $\boldsymbol{D}_{\boldsymbol{m}}, \boldsymbol{D}_{\boldsymbol{m}^{\prime}} \in \boldsymbol{D}$ is

$d\left(\boldsymbol{D}_{\boldsymbol{m}}, \boldsymbol{D}_{\boldsymbol{m}^{\prime}}\right)=\left|\bigcup_{k \in[K]: m(k) \neq m^{\prime}(k)}\left\{i \in[n]: \boldsymbol{C}_{m(k), i} \neq \boldsymbol{C}_{m^{\prime}(k), i}\right\}\right|$

Since $\boldsymbol{m} \neq \boldsymbol{m}^{\prime}$ implies there exists at least one such $k$,

$$
\begin{aligned}
d_{\min }(\boldsymbol{D}) & \geq \min _{\boldsymbol{m}, \boldsymbol{m}^{\prime}: \boldsymbol{m} \neq \boldsymbol{m}^{\prime}} \min _{k: m(k) \neq m^{\prime}(k)} d\left(\boldsymbol{C}_{m(k)}, \boldsymbol{C}_{m^{\prime}(k)}\right) \\
& =d_{\text {min }}(\boldsymbol{C}) .
\end{aligned}
$$

Combining the two sides of the argument gives

$$
d_{\min }(\boldsymbol{D})=d_{\min }(\boldsymbol{C}) .
$$

Thus $\operatorname{Pr}\left[d_{\min }(\boldsymbol{D}) \leq \gamma n\right]=\operatorname{Pr}\left[d_{\min }(\boldsymbol{C}) \leq \gamma n\right]=$ $O\left(n^{-\left(\frac{\lambda}{2}-1\right)}\right)$ gives the desired result.

\section{APPENDIX D \\ Proof of Theorem 2}

Lemma 5 of Appendix $\mathrm{C}$ shows that for $R=1-\frac{\lambda}{\rho}$ and $\lambda \geq 3$, there exist some $\gamma \in(0,1 / 2]$ for which $\operatorname{Pr}\left[d_{\min }(\boldsymbol{D}) \leq\right.$ $\gamma n]=O\left(n^{-\left(\frac{\lambda}{2}-1\right)}\right)$ under our $\operatorname{LDPC}_{K}(\lambda, \rho ; n)$ ensemble. Fix any $\sigma$ smaller than $\gamma$. We first particularize Theorem 1 to the expurgated ensemble $\operatorname{LDPC}_{K}-\operatorname{Ex}_{\sigma}(\lambda, \rho ; n)$ to bound the ensemble-average error probability $E_{\mathrm{ex}, \sigma}\left[P_{e}^{(n)}\right]$ as

$$
E_{\mathrm{ex}, \sigma}\left[P_{e}^{(n)}\right] \leq \sum_{\boldsymbol{t} \in \mathrm{T}} \bar{S}_{\mathrm{ex}, \sigma}^{n}(\boldsymbol{t}) \mathcal{D}^{t}+q^{-n E_{p}\left(K R+\left(\log \alpha_{\mathrm{MAc}}\right) / n\right)},
$$

where $E_{\text {ex, } \sigma}[\cdot]$ denotes expectation under the expurgated ensemble, $\overline{\boldsymbol{S}}_{\mathrm{ex}, \sigma}^{n}$ is the ensemble-average spectrum under the expurgated $\mathrm{LDPC}_{K}-\mathrm{Ex}_{\sigma}(\lambda, \rho ; n)$ ensemble, and

$$
\mathrm{T} \triangleq\left\{\boldsymbol{t} \in \mathcal{T}_{\mathcal{Q}}^{n}: 0<\mathrm{wt}(\boldsymbol{t}) \leq \sigma n\right\},
$$

where for all $t \in \mathcal{T}_{\mathcal{Q}}^{n}$, wt $(t)$ is the number of nonzero rows in a matrix of type $t$. 
Before bounding each of the elements in 253), we first prove that there exist some finite integer $n_{0}$ such that

$$
\begin{array}{ll}
\bar{S}_{\mathrm{ex}, \sigma}^{n}(\boldsymbol{t})=0 & \text { if } 0<\mathrm{wt}(\boldsymbol{t}) \leq \sigma n \\
\bar{S}_{\mathrm{ex}, \sigma}^{n}(\boldsymbol{t}) \leq 2 \bar{S}_{L}^{n}(\boldsymbol{t}) & \text { if } \mathrm{wt}(\boldsymbol{t})>\sigma n \text { and } n>n_{0} .
\end{array}
$$

The first property follows immediately from the definition of the expurgated code. To prove the second property, recall that $\sigma<\gamma$ by assumption. Therefore, the probability that the minimum distance of a randomly chosen code from the $\operatorname{LDPC}_{K}-\operatorname{Ex}_{\sigma}(\lambda, \rho ; n)$ ensemble is less than $\sigma n$ decays as $O\left(n^{-\left(\frac{\lambda}{2}-1\right)}\right)$. In other words, there exist constants $a \in \mathbb{R}$ and $n_{0}^{\prime} \in \mathbb{Z}$ such that for all $n>n_{0}^{\prime}$,

$$
\operatorname{Pr}\left[d_{\min }(\boldsymbol{D}) \leq \gamma n\right] \leq a n^{-\left(\frac{\lambda}{2}-1\right)} .
$$

To guarantee that the support set of the expurgated ensemble is at least half the size of the support set of the original ensemble, we choose $n$ sufficiently large so that $a n^{-\left(\frac{\lambda}{2}-1\right)} \leq \frac{1}{2}$. Under this assumption, no more than half of the support are expurgated. This gives

$$
\begin{aligned}
a n^{-\left(\frac{\lambda}{2}-1\right)} & \leq \frac{1}{2} \\
-\left(\frac{\lambda}{2}-1\right) \log (a n) & \leq \log \frac{1}{2} \\
\log (a n) & \geq\left(\frac{1}{\lambda / 2-1}\right) \log 2 \\
n & \geq \frac{q^{\frac{\log 2}{\lambda / 2-1}}}{a} .
\end{aligned}
$$

Therefore choosing $n_{0}>\max \left\{n_{0}^{\prime}, q^{\frac{\log 2}{\lambda / 2-1}} / a\right\}$ ensures that $\bar{S}_{\mathrm{ex}, \sigma}^{n}(\boldsymbol{t}) \leq 2 \bar{S}_{L}^{n}(\boldsymbol{t})$ for any $n>n_{0}$ and any $\boldsymbol{t}$ with wt $(\boldsymbol{t})>\sigma n$. While both $n_{0}^{\prime}$ and $a$ are unknown, the existence of such values proves that the desired property holds for all $n$ sufficiently large.

By (254), $\bar{S}_{\mathrm{ex}, \sigma}^{n}(\boldsymbol{t})=0$ for any $\boldsymbol{t}$ with $\operatorname{wt}(\boldsymbol{t}) \leq \sigma n$. Therefore, the term $\sum_{t \in \mathrm{T}} \bar{S}_{\mathrm{ex}, \sigma}^{n}(\boldsymbol{t}) \mathcal{D}^{t}$ in 253) equals 0 .

For the rate offset $\frac{\log \alpha_{\mathrm{MAC}}}{n}$ in the second term of 253, recall that

$$
\begin{aligned}
\mathrm{T}^{c} & =\left\{n \boldsymbol{\theta}: \boldsymbol{\theta} \in J_{\sigma}\right\} \\
\alpha_{\mathrm{MAC}} & =\max _{\boldsymbol{t} \in \mathrm{T}^{c}} \frac{\bar{S}_{\mathrm{ex}, \sigma}^{n}(\boldsymbol{t})}{\left(M^{K}-1\right) B(n, \boldsymbol{t}) q^{-n K}} \\
& =\max _{\boldsymbol{\theta} \in J_{\sigma}} \frac{\bar{S}_{\mathrm{ex}, \sigma}^{n}(\boldsymbol{t})}{\left(M^{K}-1\right) B(n, \boldsymbol{t}) q^{-n K}},
\end{aligned}
$$

$J_{\sigma}$ is defined in 242), and and $B(n, \boldsymbol{t})=n ! /\left(\prod_{g}\left(t_{g}\right) !\right)$ is the number of distinct possible codematrices of type $n \boldsymbol{\theta}$. Therefore

$$
\begin{aligned}
& \frac{1}{n} \log \alpha_{\mathrm{MAC}} \\
&=\frac{1}{n} \log \max _{\boldsymbol{\theta} \in J_{\sigma}} \frac{\bar{S}_{\mathrm{ex}, \sigma}^{n}(n \boldsymbol{\theta})}{\left(M^{K}-1\right) B(n, n \boldsymbol{\theta}) q^{-n K}} \\
&=\max _{\boldsymbol{\theta} \in J_{\sigma}}\left[\frac{1}{n} \log \bar{S}_{\mathrm{ex}, \sigma}^{n}(n \boldsymbol{\theta})-\right. \\
& \\
&\left.\quad \frac{1}{n} \log \left(\left(M^{K}-1\right) B(n, n \boldsymbol{\theta}) q^{-n K}\right)\right] \\
& \stackrel{(a)}{\leq} \max _{\boldsymbol{\theta} \in J_{\sigma}}\left[\frac{1}{n} \log \bar{S}_{\mathrm{ex}, \sigma}^{n}(n \boldsymbol{\theta})-\frac{1}{n} \log \bar{S}_{L}^{n}(n \boldsymbol{\theta})\right]
\end{aligned}
$$

$$
\begin{aligned}
& +\max _{\boldsymbol{\theta} \in J_{\sigma}}\left[\frac{1}{n} \log \bar{S}_{L}^{n}(n \boldsymbol{\theta})-\bar{S}_{L}(\boldsymbol{\theta})\right] \\
& +\max _{\boldsymbol{\theta} \in J_{\sigma}}\left[\bar{S}_{L}(\boldsymbol{\theta})-\bar{S}_{U}(\boldsymbol{\theta})\right] \\
+ & \max _{\boldsymbol{\theta} \in J_{\sigma}}\left[\bar{S}_{U}(\boldsymbol{\theta})-\frac{1}{n} \log \left(\left(M^{K}-1\right) B(n, n \boldsymbol{\theta}) q^{-n K}\right)\right],
\end{aligned}
$$

where $(a)$ follows from triangle inequality for the max function.

By (254), $\bar{S}_{\text {ex }, \sigma}^{n}(n \boldsymbol{\theta}) \leq 2 \bar{S}_{L}^{n}(n \boldsymbol{\theta})$ for all $\boldsymbol{\theta} \in J_{\sigma}$; therefore,

$$
\begin{aligned}
\max _{\boldsymbol{\theta} \in J_{\sigma}}\left[\frac{1}{n} \log \bar{S}_{\mathrm{ex}, \sigma}^{n}(n \boldsymbol{\theta})-\frac{1}{n} \log \bar{S}_{L}^{n}(n \boldsymbol{\theta})\right] & \leq \frac{\log 2}{n} \\
& =O\left(\frac{1}{n}\right) .
\end{aligned}
$$

To bound the second element in 262), note that

$$
\begin{aligned}
\frac{1}{n} \log \bar{S}_{L}^{n}(n \boldsymbol{\theta}) & =\frac{1}{n} \log \left[B(n, n \boldsymbol{\theta}) \operatorname{Pr}\left[\boldsymbol{d}_{n \boldsymbol{\theta}} \in \boldsymbol{D}\right]\right] \\
& =\frac{1}{n} \log B(n, n \boldsymbol{\theta})+\frac{1}{n} \log \operatorname{Pr}\left[\boldsymbol{d}_{n \boldsymbol{\theta}} \in \boldsymbol{D}\right]
\end{aligned}
$$

By [33. Th. 17.4.3], the multinomial coefficient can be bounded as

$$
\frac{1}{(n+1)^{q^{K}}} q^{n H(\boldsymbol{\theta})} \leq B(n, n \boldsymbol{\theta}) \leq q^{n H(\boldsymbol{\theta})} .
$$

Further, recall from [239] that $\operatorname{Pr}\left[\boldsymbol{d}_{n \boldsymbol{\theta}} \in \boldsymbol{D}\right]$ is the probability that a type- $n \boldsymbol{\theta}$ matrix $\boldsymbol{d}_{n \boldsymbol{\theta}}$ is in the codebook $\boldsymbol{D}$ of a randomly drawn code from the $\operatorname{LDPC}_{K}(\lambda, \rho ; n)$ ensemble and that

$$
\operatorname{Pr}\left[\boldsymbol{d}_{n \boldsymbol{\theta}} \in \boldsymbol{D}\right]=\frac{\left\lfloor\left((A(\boldsymbol{x}))^{n \lambda / \rho}\right)\right\rfloor_{n \lambda \boldsymbol{\theta}}}{B(n \lambda, n \lambda \boldsymbol{\theta})(q-1)^{n \lambda}} .
$$

Applying Lemma 4 to bound the numerator in 267) gives

$$
\frac{1}{n} \log \left\lfloor\left((A(\boldsymbol{x}))^{n \lambda / \rho}\right)\right\rfloor_{n \lambda \boldsymbol{\theta}} \leq \frac{\lambda}{\rho} \log \inf _{\substack{\boldsymbol{x}: \operatorname{sgn}(\boldsymbol{x}) \\=\operatorname{sgn}(\boldsymbol{\theta})}} \frac{A(\boldsymbol{x})}{\boldsymbol{x}^{\rho \boldsymbol{\theta}}} .
$$

Applying the lower bound of 266 to bound $B(n \lambda, n \lambda \boldsymbol{\theta})$, combining it with 267) and 268) yields

$$
\begin{aligned}
& \operatorname{Pr}\left[\boldsymbol{d}_{n \boldsymbol{\theta}} \in \boldsymbol{D}\right] \\
& \leq(n \lambda+1)^{q^{K}} q^{n\left(-\lambda \log (q-1)-\lambda H(\boldsymbol{\theta})+(1-R) \log \inf A(\boldsymbol{x}) / \boldsymbol{x}^{\rho \boldsymbol{\theta}}\right),}
\end{aligned}
$$

where we take the infimum in 269 over all $\boldsymbol{x}$ for which $\operatorname{sgn}(\boldsymbol{x})=\operatorname{sgn}(\boldsymbol{\theta})$.

Therefore, we obtain the bound

$$
\begin{aligned}
\frac{1}{n} \overline{\boldsymbol{S}}_{L}(n \boldsymbol{\theta}) & \leq H(\boldsymbol{\theta})+\frac{1}{n} \log (n \lambda+1)^{q^{K}}-\lambda H(\boldsymbol{\theta}) \\
& -\lambda \log (q-1)+\frac{\lambda}{\rho} \log \inf _{\boldsymbol{x}: \operatorname{sgn}(\boldsymbol{x})=\operatorname{sgn}(\boldsymbol{\theta})} \frac{A(\boldsymbol{x})}{\boldsymbol{x}^{\rho \boldsymbol{\theta}}},
\end{aligned}
$$

giving

$$
\begin{aligned}
\max _{\boldsymbol{\theta} \in J_{\sigma}}\left[\frac{1}{n} \log \bar{S}_{L}^{n}(n \boldsymbol{\theta})-\bar{S}_{L}(\boldsymbol{\theta})\right] & \leq \frac{1}{n} \log (n \lambda+1)^{q^{K}} \\
& =O\left(\frac{\log n}{n}\right) .
\end{aligned}
$$


By Theorem 18 , there exist $n$ and $\rho$ such that $\bar{S}_{L}(\boldsymbol{\theta})-$ $\bar{S}_{U}(\boldsymbol{\theta})<\epsilon$ for any $\epsilon>0$. To make the statement more precise, recall from (247) that

$$
\bar{S}_{L}(\boldsymbol{\theta})-\bar{S}_{U}(\boldsymbol{\theta})<\log \left(1+\sum_{\boldsymbol{k} \neq \mathbf{0}}(\boldsymbol{\theta}[\mathbf{0}]+\psi(1-\boldsymbol{\theta}[\mathbf{0}]))^{\rho}\right),
$$

where $\boldsymbol{\theta}[\mathbf{0}]+\psi(1-\boldsymbol{\theta}[\mathbf{0}])<1$ is some constant that depends on $q$ and $\boldsymbol{\theta}$.

Using a power series expansion on the function $\log (1+x)$ reveals that $\bar{S}_{L}(\boldsymbol{\theta})-\bar{S}_{U}(\boldsymbol{\theta})$ decreases exponentially in $\rho$. Specifically, assume that $\rho=\kappa n$, where $\kappa$ is some constant that is much smaller than $\frac{q-1}{q}$; here $\kappa$ captures the density of the LDPC code which we treat as a fixed proportion of the blocklength $n$, with low $\kappa$ yielding low density and therefore low LDPC decoding complexity and high $\kappa$ yielding improvements in LDPC code performance at the cost of higher complexity. Using this choice of $\rho$ gives

$$
\max _{\boldsymbol{\theta} \in J_{\sigma}}\left[\bar{S}_{L}(\boldsymbol{\theta})-\bar{S}_{U}(\boldsymbol{\theta})\right]=O\left(c_{0}^{\kappa n}\right)
$$

where $c_{0}<1$ is some constant that depends on $q$ and $\delta$.

To bound the final term in 262, recall that $\overline{\boldsymbol{S}}_{U}(\boldsymbol{\theta})=$ $H(\boldsymbol{\theta})-K(1-R)$ and $M=q^{n R}$. Therefore,

$$
\begin{aligned}
& \bar{S}_{U}(\boldsymbol{\theta})-\frac{1}{n} \log \left(\left(M^{K}-1\right) B(n, n \boldsymbol{\theta}) q^{-n K}\right) \\
= & H(\boldsymbol{\theta})-K(1-R) \\
& \quad-\left[\frac{1}{n} \log \left(q^{n R K}-1\right)+\frac{1}{n} \log B(n, n \boldsymbol{\theta})-K\right] \\
= & H(\boldsymbol{\theta})-\frac{1}{n} \log B(n, n \boldsymbol{\theta})+\left[K R-\frac{1}{n} \log \left(q^{n R K}-1\right)\right] \\
= & H(\boldsymbol{\theta})-\frac{1}{n} \log B(n, n \boldsymbol{\theta})+\frac{\log \left(1-1 / q^{n R K}\right)}{n} \\
= & H(\boldsymbol{\theta})-\frac{1}{n} \log B(n, n \boldsymbol{\theta})+O\left(\frac{1}{n q^{n R K}}\right),
\end{aligned}
$$

where (279) follows from the power series expansion on the function $\log (1-x)$.

Applying the following Stirling's bound on $n$ ! [34], which is valid for any positive integers $n$,

$$
\sqrt{2 \pi} n^{n+\frac{1}{2}} e^{-n} e^{\frac{1}{12 n+1}}<n !<\sqrt{2 \pi} n^{n+\frac{1}{2}} e^{-n} e^{\frac{1}{12 n}}
$$

gives the expression $\log n !=n \log n-n \log e+\frac{1}{2} \log (2 \pi n)+$ $O(1 / n)$. Therefore,

$$
\begin{aligned}
& H(\boldsymbol{\theta})-\frac{1}{n} \log B(n, n \boldsymbol{\theta}) \\
&=H(\boldsymbol{\theta})-\frac{1}{n}\left[n \log n-n \log e+\frac{1}{2} \log (2 \pi n)\right. \\
&-\sum_{g \in \mathcal{Q}: \theta_{g} \neq 0}\left(n \theta_{g} \log \left(n \theta_{g}\right)-n \theta_{g} \log e+\frac{1}{2} \log \left(2 \pi n \theta_{g}\right)\right) \\
&\left.+O\left(\frac{1}{n}\right)\right]
\end{aligned}
$$

$$
\begin{aligned}
= & H(\boldsymbol{\theta})-\frac{1}{n}\left[n \log n-\sum_{g \in \mathcal{Q}: \theta_{g} \neq 0} n \theta_{g} \log \left(n \theta_{g}\right)\right. \\
& \left.+\frac{1}{2} \log (2 \pi n)-\frac{1}{2} \log \left(\prod_{g \in \mathcal{Q}: \theta_{g} \neq 0} 2 \pi n \theta_{g}\right)+O\left(\frac{1}{n}\right)\right] \\
= & H(\boldsymbol{\theta})-\left[\log n-\sum_{g \in \mathcal{Q}: \theta_{g} \neq 0} \theta_{g} \log \left(n \theta_{g}\right)\right] \\
= & H(\boldsymbol{\theta})-\left[\frac{\log n}{n}\right)+O\left(\frac{1}{n^{2}}\right) \\
= & H(\boldsymbol{\theta})-\sum_{g \in \mathcal{Q}: \theta_{g} \neq 0}\left(\theta_{g} \log \theta_{g}\right)+O\left(\frac{\log n}{n}\right) \\
= & \left.O\left(\frac{\log n}{n}\right) . \quad \sum_{g \in \mathcal{Q}: \theta_{g} \neq 0} \theta_{g} \log \left(n \theta_{g}\right)\right]+O\left(\frac{\log n}{n}\right)
\end{aligned}
$$

Returning to 279,

$$
\begin{aligned}
& \max _{\boldsymbol{\theta} \in J_{\sigma}}\left[\bar{S}_{U}(\boldsymbol{\theta})-\frac{1}{n} \log \left(\left(M^{K}-1\right) B(n, n \boldsymbol{\theta}) q^{-n K}\right)\right] \\
= & O\left(\frac{\log n}{n}\right) .
\end{aligned}
$$

Combining 262, 264, 273, 275) and 288, gives

$$
\frac{\log \alpha_{\text {мас }}}{n}=O\left(\frac{1}{n}\right)+O\left(\frac{\log n}{n}\right)+O\left(c_{0}^{\kappa n}\right)+O\left(\frac{\log n}{n}\right),
$$

where $c_{0}<1$ is some constant that depends on $q$ and $\delta$.

To conclude, $\frac{1}{n} \log \alpha_{\text {MAC }}$ decays to zero as $O\left(\frac{\log n}{n}\right)$ for large enough $\rho$ (or, as a special case, for a constant $\kappa$ such that $\frac{q-1}{q}>\kappa>0$ and $\left.\rho=\kappa n\right)$.

Remark 20: To achieve even lower density, we can set $\rho=\kappa(n) n$ for some function $\kappa(n)$ that decays with $n$. When $\kappa(n) \rightarrow 0$ no more quickly than $\boldsymbol{\Theta}\left(\frac{\log n}{n}\right)$, we again find that $\frac{1}{n} \log \alpha_{\text {мас }}$ decays to zero as $O\left(\frac{\log n}{n}\right)$. To see this, note from 289) that

$$
\frac{1}{n} \log \alpha_{\mathrm{MAC}}=O\left(c_{0}^{\kappa(n) n}\right)+O\left(\frac{\log n}{n}\right) .
$$

To find the fastest decay rate of $\kappa(n)$ for which $O\left(c_{0}^{\kappa(n) n}\right)$ behaves as $O\left(\frac{\log n}{n}\right)$, we set

$$
\begin{aligned}
c_{0}^{\kappa(n) n} & =\frac{\log n}{n} \\
\kappa(n) n \log c_{0} & =\log \log n-\log n \\
\kappa(n) & =\frac{\log \log n-\log n}{n \log c_{0}} .
\end{aligned}
$$

Therefore, from 293 we conclude that when $\kappa(n)$ decays no more quickly than $\Theta\left(\frac{\log n}{n}\right)$, then $O\left(c_{0}^{\kappa(n) n}\right)$ does not 
dominate $O\left(\frac{\log n}{n}\right)$, which in turn makes $\frac{\log \alpha_{\text {MAC }}}{n}$ behave as $O\left(\frac{\log n}{n}\right)$.

\section{APPENDIX E \\ PROOF OF THEOREM 3}

The proof of Theorem 3 is similar to the proof of [35. Lemma 7]. For any code $C$ from the $\operatorname{LDPC}(\lambda, \rho ; n)$ ensemble before random codeword removal, we have $R_{C} \geq R$. If the expected value of the actual rate is close to the design rate, then one can apply Markov's inequality to demonstrate that most codes have rates close to the design rate.

From Theorem 17, we have for any $\boldsymbol{\theta}=(\theta(g): g \in \mathcal{Q})$

$$
\begin{aligned}
\bar{S}_{L}(\boldsymbol{\theta}) & \triangleq \lim _{n \rightarrow \infty} \frac{1}{n} \log \bar{S}_{L}^{n}(n \boldsymbol{\theta}) \\
& =(1-\lambda) H(\boldsymbol{\theta})-\lambda \log (q-1)+\frac{\lambda}{\rho} \log \inf _{\substack{\boldsymbol{x}: \operatorname{sgn}(\boldsymbol{x}) \\
=\operatorname{sgn}(\boldsymbol{\theta})}} \frac{A(\boldsymbol{x})}{\boldsymbol{x}^{\rho \boldsymbol{\theta}}} .
\end{aligned}
$$

We want to determine the expected rate $\lim _{n \rightarrow \infty} \frac{1}{n} \log \bar{S}_{\text {all }}^{n}$, where $\bar{S}_{\text {all }}^{n}=\sum_{n \boldsymbol{\theta} \in \mathcal{T}_{\mathcal{Q}}^{n}} \bar{S}_{L}^{n}(n \boldsymbol{\theta})$ is the ensemble-average number of codematrices and $\mathcal{T}_{\mathcal{Q}}^{n}$ is the set of possible types at length $n$. Since there is only a polynomial number of types $\left|\mathcal{T}_{\mathcal{Q}}^{n}\right|$ and the number of codematrices increases exponentially in $n$, the expected rate is equal to the supremum of $\bar{S}_{L}(\boldsymbol{\theta})$ over all $\boldsymbol{\theta}$. Setting up the Lagrangian of $\bar{S}_{L}(\boldsymbol{\theta})$ with the constraint $\sum_{g \in \mathcal{Q}} \theta_{g}=1$ gives

$$
\theta_{g}=\Lambda x_{g}^{\frac{\lambda}{\lambda-1}}, \forall g \in \mathcal{Q},
$$

where $\Lambda$ is a constant chosen to satisfy the constraint $\sum_{g \in \mathcal{Q}} \theta_{g}=1$. Substituting the value of $\theta_{g}$ back into $\bar{S}_{L}(\boldsymbol{\theta})$, taking its partial derivative with respect to each $x_{g}$, and using the symmetry of $\bar{S}_{L}(\boldsymbol{\theta})$ with respect to each $x_{g}$, we find that the stationary point happens when

$$
x_{g}=x_{\mathbf{0}}, \forall g \in \mathcal{Q} \text {. }
$$

Therefore

$$
\theta_{g}=\theta_{\mathbf{0}}=\frac{1}{|\mathcal{Q}|}=\frac{1}{q^{K}}, \forall g \in \mathcal{Q},
$$

and the supremum of $\bar{S}_{L}(\boldsymbol{\theta})$ is

$$
\begin{aligned}
\bar{S}_{L}(\boldsymbol{\theta})= & (1-\lambda) \log q^{K}-\lambda \log (q-1)+ \\
& \frac{\lambda}{\rho} \inf _{\substack{\boldsymbol{x} \operatorname{sgn}(\boldsymbol{x}) \\
=\operatorname{sgn}(\boldsymbol{\theta})}}\left[\log A(\boldsymbol{x})-\log \prod_{g} x_{g}^{\rho / q^{K}}\right] \\
\stackrel{(a)}{=} \quad & K(1-\lambda)-\lambda \log (q-1)+\frac{\lambda}{\rho} \underset{\substack{\boldsymbol{x}: \operatorname{sgn}(\boldsymbol{x}) \\
=\operatorname{sgn}(\boldsymbol{\theta})}}{\inf } \\
& {\left[\log \frac{(q-1)^{\rho}}{q^{K}}\left(q^{K} x_{\mathbf{0}}\right)^{\rho}-\log x_{\mathbf{0}}^{\rho}\right] } \\
= & K(1-\lambda)-\lambda \log (q-1)+\frac{\lambda}{\rho} \underset{\boldsymbol{x}: \operatorname{sgn}(\boldsymbol{x})}{=\operatorname{sgn}(\boldsymbol{\theta})} \\
& {\left[\rho \log (q-1)-K+\rho K+\rho \log x_{\mathbf{0}}-\rho \log x_{\mathbf{0}}\right] } \\
= & K(1-\lambda)-\frac{\lambda}{\rho} K+\lambda K
\end{aligned}
$$

$$
=K R,
$$

where $(a)$ follows from 294) and the fact that the DFT of a constant sequence is only non-zero at zero. That is, the components of $A(\boldsymbol{x})$ (defined in (238)) is non-zero only when $\boldsymbol{k}=\mathbf{0}$, and there are $\left(p^{m}-1\right)^{\rho}=(q-1)^{\rho}$ of such terms.

In summary, the expected rate satisfies

$$
\frac{1}{n} \log \bar{S}_{\text {all }}^{n}=K R+\omega_{n},
$$

where $\omega_{n}=o(1)$. Let $S_{D}^{n}$ denote the number of codematrices in the randomly drawn MAC codebook corresponding to the underlying LDPC code $C$. Applying Markov's inequality gives

$$
\begin{aligned}
\operatorname{Pr}\left[R_{\boldsymbol{C}} \geq R+\epsilon\right] & =\operatorname{Pr}\left[q^{n K R_{C}} \geq q^{n K R} \cdot q^{n K \epsilon}\right] \\
& =\operatorname{Pr}\left[S_{\boldsymbol{d}}^{n} \geq \bar{S}_{\text {all }}^{n} q^{n\left(\epsilon-\omega_{n}\right)}\right] \\
& \leq \frac{\mathbb{E}\left[S_{\boldsymbol{d}}^{n}\right]}{\bar{S}_{\text {all }}^{n} q^{n\left(\epsilon-\omega_{n}\right)}} \\
& \leq q^{-n \epsilon / 2}
\end{aligned}
$$

for any $\epsilon>0$ and $n \geq n(\epsilon)$, where $n(\epsilon)$ is chosen so that $\omega_{n} \leq \epsilon / 2$ for all $n \geq n(\epsilon)$. This completes the proof of the first claim (24).

To prove the second claim 25$]$, notice that $R_{C} \leq 1$, hence

$$
\begin{aligned}
\mathbb{E}\left[R_{C}-R\right] & \\
= & \mathbb{E}\left[R_{C}-R \mid R_{\boldsymbol{C}}-R \leq \epsilon\right] \cdot \operatorname{Pr}\left[R_{\boldsymbol{C}}-R \leq \epsilon\right] \\
& +\mathbb{E}\left[R_{\boldsymbol{C}}-R \mid R_{\boldsymbol{C}}-R>\epsilon\right] \cdot \operatorname{Pr}\left[R_{C}-R>\epsilon\right] \\
\leq & \epsilon \cdot 1+1 \cdot q^{-n \epsilon / 2},
\end{aligned}
$$

and the second claim follows by choosing $\epsilon=\frac{2 \log n}{n}$.

\section{APPENDIX F \\ Proof of THEOREM 4}

The proof of this theorem is very similar to the proof of Theorem 11. The key difference is that when $\left(X_{1}, X_{2}\right)=$ $\left(\boldsymbol{c}_{1,1}, \boldsymbol{c}_{2,1}\right)$ is transmitted, the set of codeword pairs for which the ML decoder fails to decode is separated into three groups:

$$
\begin{aligned}
& \left(\boldsymbol{c}_{1, i}, \boldsymbol{c}_{2,1}\right), \text { for some } i \neq 1, \\
& \left(\boldsymbol{c}_{1,1}, \boldsymbol{c}_{2, j}\right) \text {, for some } j \neq 1, \\
& \left(\boldsymbol{c}_{1, i}, \boldsymbol{c}_{2, j}\right), \text { for some }(i, j) \neq(1,1) .
\end{aligned}
$$

By the given code construction, transmitter $i$ employs a random code from the $\operatorname{LDPC}\left(\lambda_{i}, \rho_{i}, \delta_{i} ; n\right)$ ensemble. Notice that the codebook is restricted by our code design to include precisely $M_{i}=q^{n R_{i}}$ codewords for each transmitter, where the design rate $R_{i}=1-\frac{\lambda_{i}}{\rho_{i}}$ for $i \in\{1,2\}$.

Denote

$$
\boldsymbol{c}_{(i)}=\left\{\boldsymbol{c}_{i, 1}, \ldots, \boldsymbol{c}_{i, M_{i}}\right\}
$$

as the codebook for transmitter $i$ before applying coset vector and quantization. Given the coset vectors $\boldsymbol{v}_{1}$ and $\boldsymbol{v}_{2}$ and quantizers $\delta_{1}$ and $\delta_{2}$, the resulting set of channel inputs is

$\left\{\left(\delta_{1}\left(\boldsymbol{c}_{1, k_{1}}+\boldsymbol{v}_{1}\right), \delta_{2}\left(\boldsymbol{c}_{2, k_{2}}+\boldsymbol{v}_{2}\right)\right):\left(k_{1}, k_{2}\right) \in\left[M_{1}\right] \times\left[M_{2}\right]\right\}$.

For notational simplicity, let $\boldsymbol{d}=\left\{\boldsymbol{d}_{\boldsymbol{m}}: \boldsymbol{m} \in\left[M_{1}\right] \times\left[M_{2}\right]\right\}$ describe the corresponding MAC codebook; here for any $\boldsymbol{m}=$ 
$(m(1), m(2)), \boldsymbol{d}_{\boldsymbol{m}}=\left(\boldsymbol{c}_{1, m(1)}, \boldsymbol{c}_{2, m(2)}\right)$. The corresponding channel input is

$$
\boldsymbol{\delta}\left(\boldsymbol{d}_{\boldsymbol{m}}+\boldsymbol{v}\right) \triangleq\left(\delta_{1}\left(\boldsymbol{c}_{1, m(1)}+\boldsymbol{v}_{1}\right), \delta_{2}\left(\boldsymbol{c}_{2, m(2)}+\boldsymbol{v}_{2}\right)\right) .
$$

The expected value under our random code construction of the average error probability is

$$
\begin{aligned}
E\left[P_{e}^{(n)}\right] & =\sum_{\boldsymbol{m}} \sum_{\boldsymbol{d}} \sum_{\boldsymbol{v}} P_{\boldsymbol{M}}(\boldsymbol{m}) P_{\boldsymbol{D}}(\boldsymbol{d}) P_{\boldsymbol{V}}(\boldsymbol{v}) P_{e \mid \boldsymbol{m}, \boldsymbol{d}, \boldsymbol{v}}^{(n)} \\
& =E_{\boldsymbol{M} \boldsymbol{D} \boldsymbol{V}}\left[P_{e \mid \boldsymbol{M}, \boldsymbol{D}, \boldsymbol{V}}^{(n)}\right]
\end{aligned}
$$

where $P_{e \mid \boldsymbol{m}, \boldsymbol{d}, \boldsymbol{v}}^{(n)}$ is the conditional error probability under fixed values of the message vector $\boldsymbol{m}=(m(1), m(2))$, codebook $\boldsymbol{d}=\boldsymbol{c}_{(1)} \times \boldsymbol{c}_{(2)}$, and coset matrix $\boldsymbol{v}=\left(\boldsymbol{v}_{1}, \boldsymbol{v}_{2}\right), P_{\boldsymbol{M}}(\boldsymbol{m})$, $P_{\boldsymbol{D}}(\boldsymbol{d})$, and $P_{\boldsymbol{V}}(\boldsymbol{v})$ capture the (independent, uniform) distributions on the vectors of possible messages over $\left[M_{1}\right] \times\left[M_{2}\right]$, set of possible codebooks, and cosets over $\operatorname{GF}(q)^{n} \times \operatorname{GF}(q)^{n}$, respectively, and $E_{M D V}[\cdot]$ is the resulting expectation.

We begin by bounding the conditional error probability $P_{e \mid \boldsymbol{m}, \boldsymbol{d}, \boldsymbol{v}}^{(N)}$. Let

$$
\begin{aligned}
\mathcal{Y}_{\boldsymbol{m}, \boldsymbol{d}, \boldsymbol{v}}= & \left\{\boldsymbol{y}: \exists \boldsymbol{m}^{\prime} \in\left[M_{1}\right] \times\left[M_{2}\right] \backslash\{\boldsymbol{m}\}\right. \text { s.t. } \\
& \left.\operatorname{Pr}\left[\boldsymbol{y} \mid \boldsymbol{\delta}\left(\boldsymbol{d}_{\boldsymbol{m}^{\prime}}+\boldsymbol{v}\right)\right] \geq \operatorname{Pr}\left[\boldsymbol{y} \mid \boldsymbol{\delta}\left(\boldsymbol{d}_{\boldsymbol{m}}+\boldsymbol{v}\right)\right]\right\} .
\end{aligned}
$$

Then

$$
P_{e \mid \boldsymbol{m}, \boldsymbol{d}, \boldsymbol{v}}^{(n)} \leq \operatorname{Pr}\left[\mathcal{Y}_{\boldsymbol{m}, \boldsymbol{d}, \boldsymbol{v}} \mid \boldsymbol{m}, \boldsymbol{d}, \boldsymbol{v}\right],
$$

which is an inequality rather than an equality since an error is not guaranteed for the case of a tie.

The set $\mathcal{Y}_{\boldsymbol{m}, \boldsymbol{d}, \boldsymbol{v}}$ can be equivalently written as the union of the following sets

$$
\begin{aligned}
& \mathcal{Y}_{\boldsymbol{m}, \boldsymbol{d}, \boldsymbol{v}}^{1}=\left\{\boldsymbol{y}: \exists i \in\left[M_{1}\right] \backslash\{m(1)\}\right. \text { s.t. } \\
&\left.\operatorname{Pr}\left[\boldsymbol{y} \mid \boldsymbol{\delta}\left(\boldsymbol{d}_{(i, m(2))}+\boldsymbol{v}\right)\right] \geq \operatorname{Pr}\left[\boldsymbol{y} \mid \boldsymbol{\delta}\left(\boldsymbol{d}_{\boldsymbol{m}}+\boldsymbol{v}\right)\right]\right\}, \\
& \mathcal{Y}_{\boldsymbol{m}, \boldsymbol{d}, \boldsymbol{v}}^{2}=\left\{\boldsymbol{y}: \exists j \in\left[M_{2}\right] \backslash\{m(2)\}\right. \text { s.t. } \\
& \\
&\left.\operatorname{Pr}\left[\boldsymbol{y} \mid \boldsymbol{\delta}\left(\boldsymbol{d}_{(m(1), j)}+\boldsymbol{v}\right)\right] \geq \operatorname{Pr}\left[\boldsymbol{y} \mid \boldsymbol{\delta}\left(\boldsymbol{d}_{\boldsymbol{m}}+\boldsymbol{v}\right)\right]\right\}, \\
& \mathcal{Y}_{\boldsymbol{m}, \boldsymbol{d}, \boldsymbol{v}}^{12}=\left\{\boldsymbol{y}: \exists i \in\left[M_{1}\right] \backslash\{m(1)\}, j \in\left[M_{2}\right] \backslash\{m(2)\}\right. \\
&\text { s.t. } \left.\operatorname{Pr}\left[\boldsymbol{y} \mid \boldsymbol{\delta}\left(\boldsymbol{d}_{(i, j)}+\boldsymbol{v}\right)\right] \geq \operatorname{Pr}\left[\boldsymbol{y} \mid \boldsymbol{\delta}\left(\boldsymbol{d}_{\boldsymbol{m}}+\boldsymbol{v}\right)\right]\right\} .
\end{aligned}
$$

Therefore, by the union bound

$$
\operatorname{Pr}\left[\mathcal{Y}_{\boldsymbol{m}, \boldsymbol{d}, \boldsymbol{v}} \mid \boldsymbol{m}, \boldsymbol{d}, \boldsymbol{v}\right] \leq \sum_{i \in\{1,2,12\}} \operatorname{Pr}\left[\mathcal{Y}_{\boldsymbol{m}, \boldsymbol{d}, \boldsymbol{v}}^{i} \mid \boldsymbol{m}, \boldsymbol{d}, \boldsymbol{v}\right]
$$

For the first term in the summation, abbreviating $i \in\left[M_{1}\right] \backslash$ $\{m(1)\}$ to $i \neq m(1)$ and taking the expectation over $\boldsymbol{m}, \boldsymbol{d}, \boldsymbol{v}$ gives

$$
\begin{aligned}
& E\left[\operatorname{Pr}\left[\mathcal{Y}_{\boldsymbol{M}, \boldsymbol{D}, \boldsymbol{V}}^{1} \mid \boldsymbol{M}, \boldsymbol{D}, \boldsymbol{V}\right]\right] \\
& =\sum_{\boldsymbol{m}, \boldsymbol{a}, \boldsymbol{y}} \operatorname{P}_{\boldsymbol{M}}(\boldsymbol{m}) P_{\boldsymbol{D}_{\boldsymbol{m}}+\boldsymbol{V}}(\boldsymbol{a}) \operatorname{Pr}[\boldsymbol{y} \mid \delta(\boldsymbol{a})] \\
& \quad \cdot \operatorname{Pr}\left[\exists i \in\left[M_{1}\right] \backslash\{m(1)\}: \operatorname{Pr}\left[\boldsymbol{y} \mid \delta\left(\boldsymbol{D}_{(i, m(2))}+\boldsymbol{V}\right)\right]\right. \\
& \left.\quad \geq \operatorname{Pr}\left[\boldsymbol{y} \mid \delta\left(\boldsymbol{D}_{\boldsymbol{m}}+\boldsymbol{V}\right)\right] \mid \boldsymbol{D}_{\boldsymbol{m}}+\boldsymbol{V}=\boldsymbol{a}\right] \\
& =\sum_{\boldsymbol{m}, \boldsymbol{a}, \boldsymbol{y}} \operatorname{PM}_{\boldsymbol{M}}(\boldsymbol{m}) P_{\boldsymbol{D}_{\boldsymbol{m}}+\boldsymbol{V}}(\boldsymbol{a}) \operatorname{Pr}[\boldsymbol{y} \mid \delta(\boldsymbol{a})] \\
& \quad \cdot \operatorname{Pr}\left[\exists i \in\left[M_{1}\right] \backslash\{m(1)\}: \boldsymbol{D}_{(i, m(2))}+\boldsymbol{V}=\boldsymbol{a}^{\prime},\right. \\
& \left.\quad \operatorname{Pr}\left[\boldsymbol{y} \mid \delta\left(\boldsymbol{a}^{\prime}\right)\right] \geq \operatorname{Pr}[\boldsymbol{y} \mid \delta(\boldsymbol{a})] \mid \boldsymbol{D}_{\boldsymbol{m}}+\boldsymbol{V}=\boldsymbol{a}\right]
\end{aligned}
$$

$$
\begin{aligned}
& \stackrel{(a)}{\leq} \sum_{\boldsymbol{m}, \boldsymbol{a}, \boldsymbol{y}} P_{\boldsymbol{M}}(\boldsymbol{m}) P_{\boldsymbol{D}_{\boldsymbol{m}}+\boldsymbol{V}}(\boldsymbol{a}) \operatorname{Pr}[\boldsymbol{y} \mid \delta(\boldsymbol{a})] \min \left\{1, \sum_{i \neq m(1)}\right. \\
& \left.\sum_{\operatorname{Pr}\left[\boldsymbol{y} \mid \delta\left(\boldsymbol{a}^{\prime}\right)\right] \geq \operatorname{Pr}[\boldsymbol{y} \mid \delta(\boldsymbol{a})]} \operatorname{Pr}\left[\boldsymbol{D}_{(i, m(2))}+\boldsymbol{V}=\boldsymbol{a}^{\prime} \mid \boldsymbol{D}_{\boldsymbol{m}}+\boldsymbol{V}=\boldsymbol{a}\right]\right\} \\
& \stackrel{(b)}{\leq} \sum_{\boldsymbol{m}, \boldsymbol{a}, \boldsymbol{y}} P_{M}(\boldsymbol{m}) P_{\boldsymbol{D}_{\boldsymbol{m}}+\boldsymbol{V}}(\boldsymbol{a}) \operatorname{Pr}[\boldsymbol{y} \mid \delta(\boldsymbol{a})]\left(\sum_{i \neq m(1)}\right. \\
& \left.\sum_{\operatorname{Pr}\left[\boldsymbol{y} \mid \delta\left(\boldsymbol{a}^{\prime}\right)\right] \geq \operatorname{Pr}[\boldsymbol{y} \mid \delta(\boldsymbol{a})]} \operatorname{Pr}\left[\boldsymbol{D}_{(i, m(2))}+\boldsymbol{V}=\boldsymbol{a}^{\prime} \mid \boldsymbol{D}_{\boldsymbol{m}}+\boldsymbol{V}=\boldsymbol{a}\right]\right)^{\rho} \\
& \stackrel{(c)}{=} \sum_{\boldsymbol{y}, \boldsymbol{a}} P_{\boldsymbol{D}_{\mathbf{1}}+\boldsymbol{V}}(\boldsymbol{a}) \operatorname{Pr}[\boldsymbol{y} \mid \delta(\boldsymbol{a})]\left(\sum_{i \neq 1}\right. \\
& \left.\sum_{\left.\operatorname{Pr} \boldsymbol{y} \mid \delta\left(\boldsymbol{a}^{\prime}\right]\right) \geq \operatorname{Pr}[\boldsymbol{y} \mid \delta(\boldsymbol{a})]} \operatorname{Pr}\left[\boldsymbol{D}_{(i, 1)}+\boldsymbol{V}=\boldsymbol{a}^{\prime} \mid \boldsymbol{D}_{\mathbf{1}}+\boldsymbol{V}=\boldsymbol{a}\right]\right)^{\rho},
\end{aligned}
$$

where $(a)$ follows from the union bound and the bounded nature of probabilities, $(b)$ follows by a case analysis for any $\rho \in[0,1]: \min \{1, a\}=1 \leq a^{\rho}$ when $a \geq 1$, and $\min \{1, a\}=$ $a \leq a^{\rho}$ when $0 \leq a<1$; and $(c)$ follows for $\mathbf{1}=(1,1)$ by the symmetry of our random code design. Under our random code design and coset choice, for any $i \neq 1$

$$
\begin{aligned}
& \operatorname{Pr}\left[\boldsymbol{D}_{\mathbf{1}}+\boldsymbol{V}=\boldsymbol{a}, \boldsymbol{D}_{(i, 1)}+\boldsymbol{V}=\boldsymbol{a}^{\prime}\right] \\
& =\sum_{\boldsymbol{v}} \operatorname{Pr}\left[\boldsymbol{V}=\boldsymbol{v}, \boldsymbol{D}_{\mathbf{1}}=\boldsymbol{a}-\boldsymbol{v}, \boldsymbol{D}_{(i, 1)}-\boldsymbol{D}_{\mathbf{1}}=\boldsymbol{a}^{\prime}-\boldsymbol{a}\right] \\
& =q^{-2 n} \sum_{\boldsymbol{v}} \operatorname{Pr}\left[\boldsymbol{D}_{\mathbf{1}}=\boldsymbol{a}-\boldsymbol{v}, \boldsymbol{D}_{(i, 1)}-\boldsymbol{D}_{\mathbf{1}}=\boldsymbol{a}^{\prime}-\boldsymbol{a}\right] \\
& =q^{-2 n} \operatorname{Pr}\left[\boldsymbol{D}_{(i, 1)}-\boldsymbol{D}_{\mathbf{1}}=\boldsymbol{a}^{\prime}-\boldsymbol{a}\right] \\
& \stackrel{(d)}{=} \quad q^{-2 n} \operatorname{Pr}\left[\boldsymbol{C}_{1, i}-\boldsymbol{C}_{1,1}=\boldsymbol{a}^{\prime}[*, 1]-\boldsymbol{a}[*, 1]\right] \\
& \stackrel{(e)}{\leq} \quad q^{-2 n} \operatorname{Pr}\left[\boldsymbol{a}^{\prime}[*, 1]-\boldsymbol{a}[*, 1] \in \boldsymbol{C}_{(1)}\right] \\
& \cdot \operatorname{Pr}\left[\boldsymbol{C}_{1, i}-\boldsymbol{C}_{1,1}=\boldsymbol{a}^{\prime}[*, 1]-\boldsymbol{a}[*, 1]\right. \\
& \left.\mid \boldsymbol{a}^{\prime}[*, 1]-\boldsymbol{a}[*, 1] \in \boldsymbol{C}_{(1)}\right] \\
& \stackrel{(f)}{\leq} \quad q^{-2 n} \frac{\bar{S}_{1}^{n}\left(\mathcal{T}_{q}^{n}\left(\boldsymbol{a}^{\prime}[*, 1]-\boldsymbol{a}[*, 1]\right)\right)}{B\left(n, \mathcal{T}_{q}^{n}\left(\boldsymbol{a}^{\prime}[*, 1]-\boldsymbol{a}[*, 1]\right)\right)} \frac{1}{M_{1}-1} \\
& \stackrel{(g)}{\leq} q^{-2 n}\left(\alpha_{1} q^{-n}\right) \text {, }
\end{aligned}
$$

where $\bar{S}_{1}^{n}(\boldsymbol{t})$ refers to the ensemble-average number of type$\boldsymbol{t}$ codewords for transmitter $1,(d)$ follows since $\boldsymbol{D}_{(i, 1)}=$ $\left(\boldsymbol{C}_{1, i}, \boldsymbol{C}_{2,1}\right)$ and $\boldsymbol{D}_{\mathbf{1}}=\left(\boldsymbol{C}_{1,1}, \boldsymbol{C}_{2,1}\right)$, and $\boldsymbol{a}^{\prime}[*, 1] / \boldsymbol{a}[*, 1]$ refers to the first column of the $n \times 2$ codematrix $\boldsymbol{a}^{\prime} / \boldsymbol{a},(e)$ follows since the difference between two codewords is also a codeword in any linear code, and the upper bound still holds even we select $M_{1}=q^{n R_{1}}$ codewords for transmitter 1, $(f)$ follows since the number of codewords $M\left(\boldsymbol{C}_{(1)}\right)$ in the random codebook for transmitter $1 M\left(\boldsymbol{C}_{(1)}\right) \geq M_{1}=q^{n R_{1}}$ prior to our random restriction to precisely $M_{1}$ codewords, and $(g)$ follows from the definition of $\alpha_{1}$ in 36 .

Since $P_{\boldsymbol{D}_{\mathbf{1}}+\boldsymbol{V}}(\boldsymbol{a})=q^{-2 n}$ by the uniformity of random coset matrix $\boldsymbol{V}$,

$$
\operatorname{Pr}\left[\boldsymbol{D}_{(i, 1)}+\boldsymbol{V}=\boldsymbol{a}^{\prime} \mid \boldsymbol{D}_{\mathbf{1}}+\boldsymbol{V}=\boldsymbol{a}\right] \leq \alpha_{1} q^{-n} .
$$


Therefore

$$
\begin{aligned}
& E\left[\operatorname{Pr}\left[\mathcal{Y}_{\boldsymbol{M}, \boldsymbol{D}, \boldsymbol{V}}^{1} \mid \boldsymbol{M}, \boldsymbol{D}, \boldsymbol{V}\right]\right] \\
& \leq \sum_{\boldsymbol{y}, \boldsymbol{a}} q^{-2 n} \operatorname{Pr}[\boldsymbol{y} \mid \delta(\boldsymbol{a})] \\
& \cdot\left(\sum_{i=2}^{q^{n R_{1}}} \sum_{\operatorname{Pr}\left[\boldsymbol{y} \mid \delta\left(\boldsymbol{a}^{\prime}\right)\right] \geq \operatorname{Pr}[\boldsymbol{y} \mid \delta(\boldsymbol{a})]} \alpha_{1} q^{-n}\right)^{\rho} \\
& \leq \alpha_{1}^{\rho} \sum_{\boldsymbol{y}, \boldsymbol{a}} q^{-2 n} \operatorname{Pr}[\boldsymbol{y} \mid \delta(\boldsymbol{a})] \\
& \cdot\left(\left(q^{n R_{1}}-1\right) \sum_{\boldsymbol{a}^{\prime}: \operatorname{Pr}\left[\boldsymbol{y} \mid \delta\left(\boldsymbol{a}^{\prime}\right)\right] \geq \operatorname{Pr}[\boldsymbol{y} \mid \delta(\boldsymbol{a})]} q^{-n}\right)^{\rho} \\
& \leq \alpha_{1}^{\rho} q^{n R_{1} \rho} \sum_{\boldsymbol{x}_{1}, \boldsymbol{x}_{2}, \boldsymbol{y}} P_{\boldsymbol{Y} \mid \boldsymbol{X}_{1}, \boldsymbol{X}_{2}}\left(\boldsymbol{y} \mid \boldsymbol{x}_{1}, \boldsymbol{x}_{2}\right) \sum_{\boldsymbol{a}: \boldsymbol{\delta}(\boldsymbol{a})=\left(\boldsymbol{x}_{1}, \boldsymbol{x}_{2}\right)} q^{-2 n}
\end{aligned}
$$

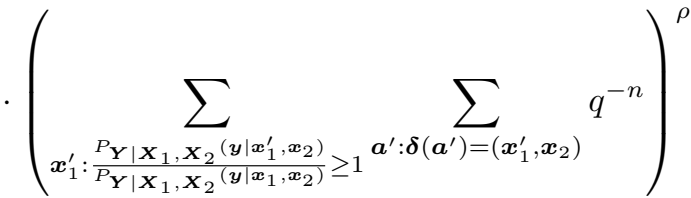

$$
\begin{aligned}
& =\alpha_{1}^{\rho} q^{n R_{1} \rho} \sum_{\boldsymbol{x}_{1}, \boldsymbol{x}_{2}, \boldsymbol{y}} P_{\boldsymbol{Y} \mid \boldsymbol{X}_{1}, \boldsymbol{X}_{2}}\left(\boldsymbol{y} \mid \boldsymbol{x}_{1}, \boldsymbol{x}_{2}\right) P_{\boldsymbol{X}_{1}}\left(\boldsymbol{x}_{1}\right) P_{\boldsymbol{X}_{2}}\left(\boldsymbol{x}_{2}\right)
\end{aligned}
$$



$$
\begin{aligned}
& \stackrel{(h)}{\leq} \alpha_{1}^{\rho} q^{n R_{1} \rho} \sum_{\boldsymbol{x}_{1}, \boldsymbol{x}_{2}, \boldsymbol{y}} P_{\boldsymbol{Y} \mid \boldsymbol{X}_{1}, \boldsymbol{X}_{2}}\left(\boldsymbol{y} \mid \boldsymbol{x}_{1}, \boldsymbol{x}_{2}\right) P_{\boldsymbol{X}_{1}}\left(\boldsymbol{x}_{1}\right) P_{\boldsymbol{X}_{2}}\left(\boldsymbol{x}_{2}\right) \\
& \cdot\left(\sum_{\boldsymbol{x}_{1}^{\prime}} P_{\boldsymbol{X}_{1}}\left(\boldsymbol{x}_{1}^{\prime}\right)\left(\frac{P_{\boldsymbol{Y} \mid \boldsymbol{X}_{1}, \boldsymbol{X}_{2}}\left(\boldsymbol{y} \mid \boldsymbol{x}_{1}^{\prime}, \boldsymbol{x}_{2}\right)}{P_{\boldsymbol{Y} \mid \boldsymbol{X}_{1}, \boldsymbol{X}_{2}}\left(\boldsymbol{y} \mid \boldsymbol{x}_{1}, \boldsymbol{x}_{2}\right)}\right)^{s}\right)^{\rho} \\
& =\alpha_{1}^{\rho} q^{n R_{1} \rho} \sum_{\boldsymbol{y}} \sum_{\boldsymbol{x}_{2}} P_{\boldsymbol{X}_{2}}\left(\boldsymbol{x}_{2}\right) \\
& \cdot\left(\sum_{\boldsymbol{x}_{1}} P_{\boldsymbol{X}_{1}}\left(\boldsymbol{x}_{1}\right) P_{\boldsymbol{Y} \mid \boldsymbol{X}_{1}, \boldsymbol{X}_{2}}\left(\boldsymbol{y} \mid \boldsymbol{x}_{1}, \boldsymbol{x}_{2}\right)^{1-s \rho}\right) \\
& \cdot\left(\sum_{\boldsymbol{x}_{1}^{\prime}} P_{\boldsymbol{X}_{1}}\left(\boldsymbol{x}_{1}^{\prime}\right) P_{\boldsymbol{Y} \mid \boldsymbol{X}_{1}, \boldsymbol{X}_{2}}\left(\boldsymbol{y} \mid \boldsymbol{x}_{1}^{\prime}, \boldsymbol{x}_{2}\right)^{s}\right)^{\rho},
\end{aligned}
$$

where $(h)$ holds for any $s>0$.

When $s=1 /(1+\rho)$, rewriting the result (296) in an exponential form using error exponent from 30p and (33), and optimizing over $0 \leq \rho \leq 1$ gives

$$
E\left[\operatorname{Pr}\left[\mathcal{Y}_{\boldsymbol{M}, \boldsymbol{D}, \boldsymbol{V}}^{1} \mid \boldsymbol{M}, \boldsymbol{D}, \boldsymbol{V}\right]\right] \leq q^{-n E_{p_{1}}\left(R_{1}+\frac{\log \alpha_{1}}{n}\right)} .
$$

Switching the role of transmitter 1 and transmitter 2 in the above proof, we obtain

$$
E\left[\operatorname{Pr}\left[\mathcal{Y}_{\boldsymbol{M}, \boldsymbol{D}, \boldsymbol{V}}^{2} \mid \boldsymbol{M}, \boldsymbol{D}, \boldsymbol{V}\right]\right] \leq q^{-n E_{p_{2}}\left(R_{2}+\frac{\log \alpha_{2}}{n}\right)} .
$$

Finally, $E\left[\operatorname{Pr}\left[\mathcal{Y}_{\boldsymbol{M}, \boldsymbol{D}, \boldsymbol{V}}^{12} \mid \boldsymbol{M}, \boldsymbol{D}, \boldsymbol{V}\right]\right]$ can be bounded using the same technique as the proof of Theorem 11, giving

$$
E\left[\operatorname{Pr}\left[\mathcal{Y}_{\boldsymbol{M}, \boldsymbol{D}, \boldsymbol{V}}^{12} \mid \boldsymbol{M}, \boldsymbol{D}, \boldsymbol{V}\right]\right] \leq q^{-n E_{p_{12}}\left(R_{1}+R_{2}+\frac{\log \alpha_{1} \alpha_{2}}{n}\right)} .
$$

Plugging the three expressions above into 295 completes the proof.

\section{APPENDIX G}

PROOF OF THEOREM 8

Given a DM-PPC $\left(\mathcal{X}, P_{Y \mid X}(y \mid x), \mathcal{Y}\right)$ with capacity achieving distribution $P_{X}$ and capacity $C$, Gallager's error exponent is defined as

$$
E_{p}(R) \triangleq \max _{0 \leq \rho \leq 1}\left[E_{0}\left(\rho, P_{X}\right)-\rho R\right]
$$

where

$$
E_{0}\left(\rho, P_{X}\right) \triangleq-\log _{e} \sum_{y \in \mathcal{Y}}\left[\sum_{x \in \mathcal{X}} P_{X}(x) P_{Y \mid X}(y \mid x)^{1 /(1+\rho)}\right]^{1+\rho},
$$

Applying a second-order Taylor expansion to $E_{0}\left(\rho, P_{X}\right)$ at $\rho=0$ gives

$$
E_{0}\left(\rho, P_{X}\right)=E_{0}\left(0, P_{X}\right)+\rho E_{0}^{\prime}\left(0, P_{X}\right)+\frac{\rho^{2}}{2} E_{0}^{\prime \prime}\left(\rho *, P_{X}\right)
$$

for some $\rho * \in[0, \rho]$.

Direct calculation gives $E_{0}\left(0, P_{X}\right)=-\log 1=0$, and $[6$ Eq. 5.5.30] shows $E_{0}^{\prime}\left(0, P_{X}\right)=C$.

Let $\beta$ be an upper bound for $-E_{0}^{\prime \prime}\left(\rho *, P_{X}\right)$. Then 301 becomes

$$
\begin{aligned}
E_{0}\left(\rho, P_{X}\right) & =0+\rho C+\frac{\rho^{2}}{2} E_{0}^{\prime \prime}\left(\rho *, P_{X}\right) \\
& \geq \rho C-\frac{\rho^{2}}{2} \beta .
\end{aligned}
$$

Therefore,

$$
E_{p}(R) \geq \rho C-\frac{\rho^{2}}{2} \beta-\rho R .
$$

The right-hand side of (304) is a concave quadratic function in $\rho$. Taking its derivative and equating the derivative to 0 yields the following stationary point

$$
\rho=\frac{C-R}{\beta},
$$

giving

$$
\begin{aligned}
E_{p}(R) & \geq \frac{C-R}{\beta} C-\left(\frac{C-R}{\beta}\right)^{2} \frac{\beta}{2}-\frac{C-R}{\beta} R \\
& =\frac{(C-R)^{2}}{2 \beta}, \text { for } C-R \leq \beta .
\end{aligned}
$$

Following the outline in [6, Exercise 5.23], one can show that (proof omitted)

$$
-E_{0}^{\prime \prime}\left(\rho, P_{X}\right) \leq \frac{4}{e^{2}}+\log _{e}^{2}|\mathcal{Y}|-\left[E_{0}^{\prime}\left(\rho, P_{X}\right)\right]^{2} .
$$

Note that $E_{0}\left(\rho, P_{X}\right)$ has the following properties ([6 Th.5.6.3])

$$
\begin{aligned}
& E_{0}\left(\rho, P_{X}\right) \geq 0, \rho \geq 0 \\
& E_{0}^{\prime}\left(\rho, P_{X}\right)>0, \rho \geq 0 \\
& E_{0}^{\prime \prime}\left(\rho, P_{X}\right) \leq 0, \rho \geq 0 .
\end{aligned}
$$


Therefore,

$$
\min _{\rho \in[0,1]} E_{0}^{\prime}\left(\rho, P_{X}\right)=E_{0}^{\prime}\left(1, P_{X}\right),
$$

and $R_{c r} \triangleq E_{0}^{\prime}\left(1, P_{X}\right)$ is known as the critical rate [6. Eq. (5.6.30)].

Plugging $R_{c r}$ into $(308)$ gives

$$
-E_{0}^{\prime \prime}\left(\rho, P_{X}\right) \leq \frac{4}{e^{2}}+\log _{e}^{2}|\mathcal{Y}|-R_{c r}^{2} .
$$

The bound in 307) requires $C-R \leq \beta$ so that $\rho$ is within $[0,1]$. This means that $\beta$ can be taken as

- $\beta=\frac{4}{e^{2}}+\log _{e}^{2}|\mathcal{Y}|$, which is valid for all $0 \leq R \leq C$, or

- $\beta=\frac{4}{e^{2}}+\log _{e}^{2}|\mathcal{Y}|-R_{c r}^{2}$, which is valid for $\max \{0, C-$ $\left.\left(\frac{4}{e^{2}}+\log _{e}^{2}|\mathcal{Y}|-R_{c r}^{2}\right)\right\} \leq R \leq C$.

Finally, substituting $\beta=\frac{4}{e^{2}}+2 \log _{e}^{2}|\mathcal{Y}|$ (which is looser than the conservative value $\frac{4}{e^{2}}+\log _{e}^{2}|\mathcal{Y}|$ ) into (307) gives a lower bound on $E_{p}(R)$. Invoking Theorem 7 with this lower bound completes the proof.

\section{REFERENCES}

[1] R. Gallager, "Low-density parity-check codes," IEEE Trans. Inf. Theory, vol. 8, no. 1, pp. 21-28, 1962.

[2] C. Berrou, A. Glavieux, and P. Thitimajshima, "Near Shannon limit error-correcting coding and decoding: Turbo-codes. 1," in Proc. 93rd IEEE Int. Conf. Comm., vol. 2, 1993, pp. 1064-1070.

[3] T. Richardson and S. Kudekar, "Design of low-density parity check codes for 5 g new radio," IEEE Comm. Mag., vol. 56, no. 3, pp. 28-34, 2018.

[4] A. Bennatan and D. Burshtein, "On the application of LDPC codes to arbitrary discrete-memoryless channels," IEEE Trans. Inf. Theory, vol. 50, no. 3, pp. 417-438, March 2004.

[5] Y. Polyanskiy, H. V. Poor, and S. Verdu, "Channel coding rate in the finite blocklength regime," IEEE Trans. Inf. Theory, vol. 56, no. 5, pp. 2307-2359, May 2010.

[6] R. Gallager, Information Theory and Reliable Communication. Springer, 1968, vol. 2.

[7] M. C. Davey and D. J. C. MacKay, "Low density parity check codes over gf(q)," in Proc. IEEE Inf. Theory Workshop, June 1998, pp. 70-71.

[8] U. Erez and G. Miller, "The $\mathrm{ml}$ decoding performance of LDPC ensembles over z/sub q/," IEEE Trans. Inf. Theory, vol. 51, no. 5, pp. 1871-1879, May 2005.

[9] C. Di, D. Proietti, I. E. Telatar, T. Richardson, and R. Urbanke, "Finitelength analysis of low-density parity-check codes on the binary erasure channel," IEEE Trans. Inf. Theory, vol. 48, no. 6, pp. 1570-1579, June 2002.

[10] T. Richrdson, A. Shokrollahi, and R. Urbanke, "Finite-length analysis of various low-density parity-check ensembles for the binary erasure channel," in Proc. IEEE Int. Symp. on Information Theory (ISIT), June 2002 , p. 1.

[11] A. Amraoui, R. Urbanke, and A. Montanari, "Finite-length scaling of irregular LDPC code ensembles," in Proc. IEEE Inf. Theory Workshop, Aug 2005, pp. 5-10.

[12] A. Amraoui, A. Montanari, T. Richardson, and R. Urbanke, "Finitelength scaling for iteratively decoded LDPC ensembles," IEEE Trans. Inf. Theory, vol. 55, no. 2, pp. 473-498, Feb 2009.

[13] R. Yazdani and M. Ardakani, "Waterfall performance analysis of finitelength LDPC codes on symmetric channels," IEEE Trans. Comm. vol. 57, no. 11, pp. 3183-3187, Nov 2009.

[14] Z. Mei, K. Cai, and G. Song, "Performance analysis of finite-length LDPC codes over asymmetric memoryless channels," IEEE Trans. Veh. Technol., vol. 68, no. 11, pp. 11338-11342, Nov 2019.

[15] E. Yang and J. Meng, "New nonasymptotic channel coding theorems for structured codes," IEEE Trans. Inf. Theory, vol. 61, no. 9, pp. 4534 4553, Sep. 2015.

[16] A. Roumy and D. Declercq, "Characterization and optimization of LDPC codes for the 2-user Gaussian multiple access channel," EURASIP J. Wirel. Comm. Netw., vol. 2007, no. 1, p. 074890, Jun 2007. [Online]. Available: https://doi.org/10.1155/2007/74890
[17] S. Sharifi, A. K. Tanc, and T. M. Duman, "LDPC code design for the two-user Gaussian multiple access channel," IEEE Trans. on Wirel. Comm., vol. 15, no. 4, pp. 2833-2844, 2015.

[18] H. Yagi and H. V. Poor, "Coset codes for compound multiple access channels with common information," IEEE Trans. Inf. Theory, vol. 57, no. 6, pp. 3429-3448, 2011.

[19] M. Ebrahimi, F. Lahouti, and V. Kostina. Two-layer coded channel access with collision resolution: Design and analysis. [Online]. Available: https://arxiv.org/abs/1909.00065

[20] V. Y. F. Tan and O. Kosut, "On the dispersions of three network information theory problems," IEEE Trans. Inf. Theory, vol. 60, no. 2, pp. 881-903, Feb 2014

[21] P. Elias, "Coding for noisy channels," in IRE Conv. Rec., vol. 3, Mar. 1955 , pp. 37-46.

[22] A. Bennatan and D. Burshtein, "Design and analysis of nonbinary LDPC codes for arbitrary discrete-memoryless channels," IEEE Trans. Inf. Theory, vol. 52, no. 2, pp. 549-583, Feb 2006.

[23] D. J. Costello, L. Dolecek, T. E. Fuja, J. Kliewer, D. G. M. Mitchell, and R. Smarandache, "Spatially coupled sparse codes on graphs: theory and practice," IEEE Comm. Mag., vol. 52, no. 7, pp. 168-176, 2014.

[24] R. C. Yavas, M. Effros, and V. Kostina. Gaussian multiple and random access in the finite blocklength regime. [Online]. Available: https://arxiv.org/abs/2001.03867

[25] Y.-S. Liu and B. L. Hughes, "A new universal random coding bound for the multiple-access channel," IEEE Trans. Inf. Theory, vol. 42, no. 2, pp. 376-386, 1996.

[26] T. Han and K. Kobayashi, "A new achievable rate region for the interference channel," IEEE Trans. Inf. Theory, vol. 27, no. 1, pp. 49-60, January 1981.

[27] S. Chen, M. Effros, and V. Kostina. Lossless source coding in the point-to-point, multiple access, and random access scenarios. [Online]. Available: https://arxiv.org/abs/1902.03366

[28] W. Feller, An Introduction to Probability Theory and Its Applications. John Wiley \& Sons, 1971, vol. 2.

[29] I. G. Shevtsova, "On the absolute constants in the Berry-Esseen-type inequalities," Doklady Mathematics, vol. 89, no. 3, pp. 378-381, May 2014. [Online]. Available: https://doi.org/10.1134/S1064562414030338

[30] Y. Huang and P. Moulin, "Finite blocklength coding for multiple access channels," in Proc. IEEE Int. Symp. on Information Theory (ISIT), July 2012, pp. 831-835.

[31] N. Shulman and M. Feder, "Random coding techniques for nonrandom codes," IEEE Trans. Inf. Theory, vol. 45, no. 6, pp. 2101-2104, Sep. 1999.

[32] D. Burshtein and G. Miller, "Asymptotic enumeration methods for analyzing LDPC codes," IEEE Trans. Inf. Theory, vol. 50, no. 6, pp. 1115-1131, June 2004.

[33] T. M. Cover and J. A. Thomas, Elements of Information Theory. John Wiley \& Sons, 2012.

[34] H. Robbins, "A remark on Stirling's formula," The American Mathematical Monthly, vol. 62, no. 1, pp. 26-29, 1955. [Online]. Available: http://www.jstor.org/stable/2308012

[35] C. Measson, A. Montanari, and R. Urbanke, "Maxwell's construction: the hidden bridge between maximum-likelihood and iterative decoding," IEEE Trans. Inf. Theory, pp. 225-, June 2004. 